\title{
In-vivo observations on stimulus-T interval, repolarization, and refractoriness
}

Citation for published version (APA):

Leerssen, H. (2000). In-vivo observations on stimulus-T interval, repolarization, and refractoriness: clinical implications for antitachycardia pacing. [Doctoral Thesis, Maastricht University]. Datawyse / Universitaire Pers Maastricht. https://doi.org/10.26481/dis.20001103hl

Document status and date:

Published: 01/01/2000

DOI:

$10.26481 /$ dis.20001103hl

Document Version:

Publisher's PDF, also known as Version of record

\section{Please check the document version of this publication:}

- A submitted manuscript is the version of the article upon submission and before peer-review. There can be important differences between the submitted version and the official published version of record.

People interested in the research are advised to contact the author for the final version of the publication, or visit the DOI to the publisher's website.

- The final author version and the galley proof are versions of the publication after peer review.

- The final published version features the final layout of the paper including the volume, issue and page numbers.

Link to publication

\footnotetext{
General rights rights.

- You may freely distribute the URL identifying the publication in the public portal. please follow below link for the End User Agreement:

www.umlib.nl/taverne-license

Take down policy

If you believe that this document breaches copyright please contact us at:

repository@maastrichtuniversity.nl

providing details and we will investigate your claim.
}

Copyright and moral rights for the publications made accessible in the public portal are retained by the authors and/or other copyright owners and it is a condition of accessing publications that users recognise and abide by the legal requirements associated with these

- Users may download and print one copy of any publication from the public portal for the purpose of private study or research.

- You may not further distribute the material or use it for any profit-making activity or commercial gain

If the publication is distributed under the terms of Article $25 \mathrm{fa}$ of the Dutch Copyright Act, indicated by the "Taverne" license above, 


$$
\begin{aligned}
& \text { MG } \\
& \text { EBA } \\
& \text { quY }
\end{aligned}
$$

In-Vivo Observations on Stimulus-T Interval,

Repolarization, and Refractoriness: Clinical Implications for Antitachycardia Pacing 
(C) H.M. Leerssen, Maastricht 2000

ISBN 9056810847

Vormgeving omslag: Grafisch Connect - René van Doorn, Gerard Kruithof Vormgeving binnenwerk: Unigraphic, Universiteit Maastricht

Druk: Datawyse / Universitaire Pers Maastricht 
In-Vivo Observations on Stimulus-T Interval, Repolarization, and Refractoriness: Clinical Implications for Antitachycardia Pacing

Proefschrift

ter verkrijging van de graad van doctor

aan de Universiteit Maastricht,

op gezag van de Rector Magnificus,

Prof. dr. A.C. Nieuwenhuijzen Kruseman,

volgens het besluit van het College van Decanen,

in het openbaar te verdedigen

op vrijdag 3 november 2000 om 12.00 uur

door

Henny Leerssen 
Promotor:

Prof. dr. H.J.J. Wellens

Co-promotor:

Dr. M.A. Vos

Beoordelingscommissie:

Prof. dr. M.A. Allessie (voorzitter)

Prof, dr. ir. M.G.J. Arts (Technische Universiteit Eindhoven)

Prof. dr. L.J. Jordaens (Universiteit van Rotterdam)

Prof. dr. R.S. Reneman

[ 4 ]

Prof. dr. H.J. Trappe (Universiteit van Bochum, Duitsland)

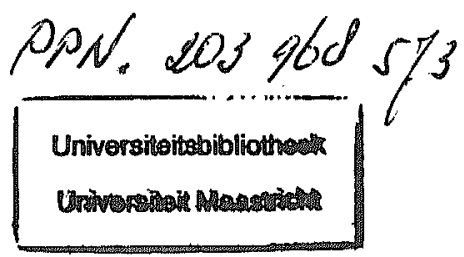

Financial support by the Netherlands Heart Foundation and the Stichting Hartsvrienden RESCAR Maastricht for the publication of this thesis is gratefully acknowledged. 
Aan Mam en Pap Voor Marlie, Casper en Eeke 
[6]

Address for Correspondence:

St. Jude Medical Nederland B.V.

P.O. Box 8005

3900 CA Veenendaal 


\section{Table of Contents}

9 Chapter 1

Introduction

29 Chapter 2

Applicability of the Stimulus-T Interval for Antitachycardia Pacing

Karel den Dulk, MD, PhD; Hendrik M. Leerssen, BS; Marc A. Vos, PhD; Willem R.M. Dassen, PhD; Jan Kersemakers; Malcolm J. Begemann, MS; Hein J.J. Wellens, MD, PhD. Published in PACE 1991; 14(Pt. II):1757-1761.

37 Chapter 3

Is the Ventricular Effective Refractory Period Different when Determined by Incremental Versus Decremental Scanning?: The Effect of Pacing Cycle-Length, dSotalol and Levcromakalim

Hendrik M. Leerssen, BS; Marc A. Vos, PhD; Karel den Dulk, MD, PhD; Jolanda van der Zande, BS; Hein J.J. Wellens, MD, PhD. Published in PACE 1994; 17(Pt. II):2084-2089.

47 Chapter 4

Inter-and Intraindividual Variations in Shortening of Ventricular Effective Refractory Period After an Abrupt Decrease in Pacing Cycle-Length

Hendrik M. Leerssen, BS; Marc A. Vos, PhD; Karel clen Dulk, MD, PhD; Jolanda van der Zande, BS; Malcolm J. Begenann, MS; Hein J.J. Wellens, MD, PhD. Published in PACE 1994; 17(Pt. II):2079-2083.

\section{Chapter 5}

Steady-State and Dynamic Behavior of Ventricular Repolarization and

Refractoriness in the Dog: The Effect of Multiple Cycle-Length Changes and

d-Sotalol Administration

Hendrik M. Leerssen, BS; Marc A. Vos, PhD; Karel den Dulk, MD, PhD; Jolanda van cler Zancle, BS; Arno M.M. Muijijens, PhD; Malcolm J. Begemann, MS; Hein J.J. Wellens, MD, PhD. Published in PACE 1998; 21:1766-1777. 


\section{Chapter 6}

Rate-Dependent Effects of Procainamide on the Threshold Current for Pacing in the Setting of Postrepolarization Refractoriness in Dogs

Hendrik M. Leerssen, BS; Marc A. Vos, PhD; Karel den Dulk, MD, PhD; Jolanda van der Zande, BS; Hein J.J. Wellens, MD, PhD. Published in PACE 1999; 22:291-301.

95 Chapter 7

High Uniformity of Left and Right Ventricular Repolarization Dynamics Induced by an Abrupt Decrease in Pacing Cycle-Length in a Dog is not Affected by Left-Ventricular Ischemia

Hendrik M. Leerssen, BS; Marc A. Vos, PhD; Richard Houben, BS; Karel den Dulk, MD, PhD; Hein J.J. Wellens, MD, PhD. Published in J Cardiovasc Electrophysiol $2000 ; 11: 421-429$.

111 Ischemia, Heart Rate, and Ventricular Repolarization

Editorial by Michiel J. Janse, MD, PhD. Published in J Cardiovasc Electrophysiol 2000; 11:430-431.

\section{Chapter 8}

Clinical Application of the Stimulus-T Interval for Antitachycardia Pacing Hendrik M. Leerssen, BS; Joep L.R.M. Smeets, MD, PhD; Marc A. Vos, PhD; Volkert A. Zeylemaker, MS; Karel den Dulk, MD, PhD; Hein J.J. Wellens, MD, PhD. Manuscript submitted. 2000.

133 Chapter 9

General Discussion

141 Summary

145 Samenvatting

149 Dankwoord

153 Curriculum Vitae

155 Publications 


\section{Chapter 1}

\section{Introduction}

\section{Table of Contents}

Treatment of Ventricular Tachyarrhythmias: Historical Perspective Antitachycardia Pacing

Aim of the Thesis

Methodological Considerations

References 


\section{Treatment of Ventricular Tachyarrhythmias: Historical Perspective}

\section{Drug Treatment}

The first electrocardiographic description of ventricular tachycardia (VT) is attributed to Thomas Lewis. In 1909 he described a patient with shortness of breath, precordial pain, and dropsy in whom he observed three to eleven "successive extrasystoles". 1 Since these ventricular arthythmias often were symptomatic, treatment was indicated. In 1922 Scott et al. demonstrated the effective termination and prevention of VT after the administration of quinidine. They hypothesized that quinidine abolished VT by lengthening of the refractory period. ${ }^{2}$ Despite its side effects such as nausea, dizziness and weakness, quinidine was used increasingly and often with good effect in the years thereafter. In 1950 pro-

[10] cainamide was used for the first time to terminate VT in man. Since side effects were less severe than for quinidine, procainamide rapidly achieved widespread acceptance. 3,4 However, in some patients, long term administration of procainamide resulted in lupus erythematodes limiting its use to short term administration. Another 10 years later lidocaine achieved widespread use as a drug of choice in treating and preventing VT in the setting of ischemic heart disease, particularly in the acute phase. Other antiarrhythmic drugs quickly followed procainamide and lidocaine (Table 1.1).

Although antiarrhythmic drugs were successfully used to terminate and prevent recurrences of VT this therapy could also facilitate the occurrence of ventricular arrhythmias. This so called proarrhythmic effect could lead to aggravation of existing VTs degenerating into ventricular fibrillation (VF), or the initiation of new VTs. As a result patients continued to die suddenly. Therefore alternative therapies had to be developed.

\section{High-Voltage Therapy}

After extensive experimental work ${ }^{5,6}$ Beck et al. ${ }^{7}$ reported in 1947 the defibrillation of a 14-year-old boy with alternating current (AC) who developed VF during a thoracic surgical procedure. The current was applied directly to the heart with two electrodes. The first transthoracic ventricular defibrillations were reported by Zoll et al. in 1956.8 They defibrillated 4 patients with AC shocks after cardiac arrest. General acceptance of this high voltage therapy was achieved after the introduction of direct current (DC) shocks, which showed to be safer and more effective than $A C$ shocks. ${ }^{-11}$ Coronary care units and later ambulances were instrumented with these external DC shock devices. Synchronization of the electrical shock to the R-wave further reduced the chance of acceleration or degeneration of the arrhythmia. Nevertheless, these life-saving DC shocks could only be applied in the presence of qualified personnel.

It lasted more than a decade before the electrical circuitry and components required for cardioversion and defibrillation could be stored in a device small enough to be implanted in a human. In 1980 the first defibrillator was implanted 
Table 1.1 Historical landmarks in the treatment of ventricular tachyarrhythmias.

\begin{tabular}{|c|c|c|c|c|c|c|c|}
\hline & acute treatement & & & chronic tre & tment & & \\
\hline & drugs & $\begin{array}{l}\text { low } \\
\text { voltage }\end{array}$ & $\begin{array}{l}\text { high } \\
\text { voltage }\end{array}$ & drugs & $\begin{array}{l}\text { low } \\
\text { voltage }\end{array}$ & $\begin{array}{l}\text { high } \\
\text { voltage }\end{array}$ & $\begin{array}{l}\text { surgery - } \\
\text { ablation }\end{array}$ \\
\hline 1922 & quinidine ${ }^{2}$ & & & quinidine ${ }^{2}$ & & & \\
\hline 1947 & & & $\begin{array}{l}\text { epicardial AC } \\
\text { shock }^{7}\end{array}$ & & & & \\
\hline 1950 & procainamide $\mathrm{e}^{3,4}$ & & & & & & \\
\hline 1.956 & & & $\begin{array}{l}\text { transthoracic } \\
A C \text { shock }\end{array}$ & & & & \\
\hline 1959 & & & & & & & $\begin{array}{l}\text { aneurysmec- } \\
\text { tomy }\end{array}$ \\
\hline 1960 & lidocaine $e^{29}$ & $\begin{array}{l}\text { overdrive } \\
\text { pacing }^{13,14}\end{array}$ & & & & & \\
\hline 1962 & & & $\begin{array}{l}\text { synchronized } \\
\text { DC shock }\end{array}$ & & & & \\
\hline 1972 & & & & $P E S^{21}$ & & & \\
\hline 7974 & & & & & & & ventriculotomy ${ }^{25}$ \\
\hline 1977 & & & & & & & cryosurgery ${ }^{26}$ \\
\hline 1980 & & & & & & $1 C D^{12}$ & $\begin{array}{l}\text { endocardial } \\
\text { resection }\end{array}$ \\
\hline 1986 & & & & & & & RF ablation \\
\hline 1989 & & & & & $I C D$ & th ATP & \\
\hline
\end{tabular}

Abbreviations: $A C=$ alternating current; $D C=$ direct current; $P E S=$ programmed electrical stimulation; $I C D=$ implantable cardioverter defibrillator; $A T P=$ antitachycardia pacing; $R F=$ radiofrequency.

in a patient. ${ }^{12}$ This device automatically delivered defibrillation therapy after detection of the arrhythmia (Table 1.1).

\section{Low-Voltage Therapy}

In 1960, Zoll et al. reported the prevention of recurrent ventricular tachyarrhythmias in 5 patients by closed chest cardiac stimulation. By pacing the heart above a certain critical rate, they could demonstrate prevention of tachyarrhythmias for periods of up to 48 hours. ${ }^{13}$ In the same year similar short term benefit from transvenous right ventricular endocardial pacing was described in patients with "bursts of ventricular fibrillation" and complete heart block.14 In 1964 Sowton et al. ${ }^{15}$ applied overdrive pacing in patients with VT and VF without evi- 
dence of heart block and they suggested that the combined use of antiarrhythmic drugs and overdrive pacing might lead to better results than either modality alone (Table 1.1).

\section{The Role of Intracardiac Recordings}

In 1945 Lenègre et al. ${ }^{16}$ were the first to record the electrical activity from within the heart. His-bundle electrograms were described 15 years later, ${ }^{17}$ but it lasted until 1969 before a safe percutaneous method for His bundle recording was described by Scherlag et al. ${ }^{18}$ After introduction of a technique to evaluate supraventricular arrhythmias by a transvenous route in 1967,19,20 Wellens et al.21 reported in 1972 the initiation and termination of VT in patients with an old myocardial infarction using programmed electrical stimulation. The ability to

[12] reproducibly initiate and terminate arrhythmias under controlled circumstances seemed to be a unique tool to evaluate the efficacy of antiarrhythmic drugs for acute treatment or prevention of the tachyarrhythmias. Furthermore, the mechanism responsible for the tachycardia and its site of origin could be diagnosed and the treatment could be chosen accordingly. The work of Wellens et al. ${ }^{21}$ has been the basis for the development of modern antiarrhythmic therapies like antiarrhythmic heart surgery, catheter ablation, antitachycardia pacing (ATP) and antiarrhythmic drug testing (Table 1.1).

\section{Surgical Treatment and Radiofrequency Ablation}

The aim of surgical treatment is to remove or to isolate the arrhythmogenic substrate. In 1959 Couch et al. ${ }^{22}$ described the elimination of VI by resection of a ventricular aneurysm in a male patient. However, the results of aneurysmectomy in larger series of patients were disappointing and the procedure was associated with a high surgical mortality of $20-50 \% .23,24$ In the years thereafter new surgical techniques such as ventriculotomy, ${ }^{25}$ cryosurgery, ${ }^{26}$ and endocardial resection $^{27}$ were introduced. However, due to the high risk of these procedures, surgical intervention was limited to patients who suffered from drug-resistant and device-refractory malignant arrhythmias. Radiofrequency ablation was first used for ablation of an accessory pathway in humans by Borggrefe et al. ${ }^{28}$ in 1986. The same group performed the first radiofrequency ablation of a VT in a patient the same year (Table 1.1).

\section{Antitachycardia Pacing}

\section{Theoretical Background}

Three mechanisms can be identified as the cause of VT: abnormal automaticity, triggered activity, and reentry. ${ }^{30}$ Evidence that arrhythmias in the chronic phase of myocardial infraction are based on reentry is quite extensive. $21,31-35$ The aim of ATP for reentrant tachycardias is to deliver a critically-timed impulse 
which invades the reentry circuit ${ }^{36,37}$ and creates refractoriness ahead of the circulating impulse (Figure 1.1).

The critical interval of premature stimuli able to do so, is referred to as the tachycardia termination window. This window starts at the end of refractoriness and ends with the longest premature beat interval at which ATP can be successful. If the stimulus is delivered just before that of the reentrant rhythm, the wavefront that starts at the site of stimulation will collide with the wavefront coming from the reentry circuit (Figure 1.1: zone 1 = collision zone). A stimulus delivered later, will fall in the absolute refractory period. It will not capture because the site of stimulation has been depolarized by the wavefront coming from the reentry cir-

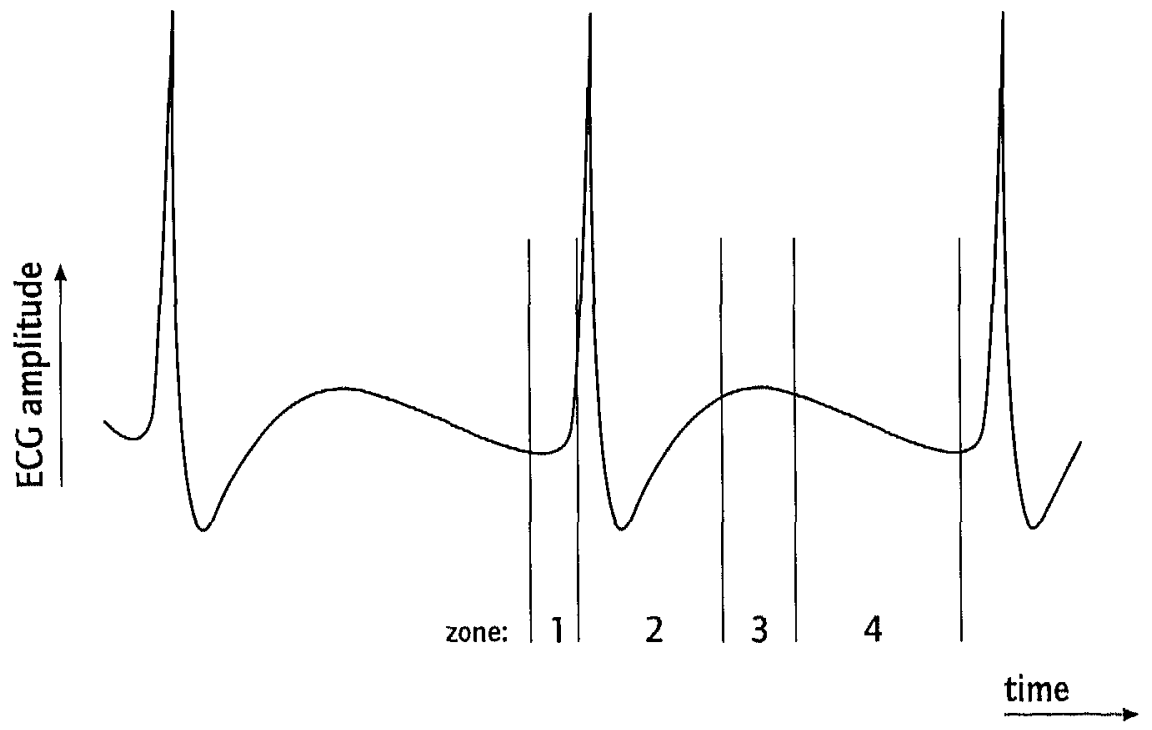

site of stimulation

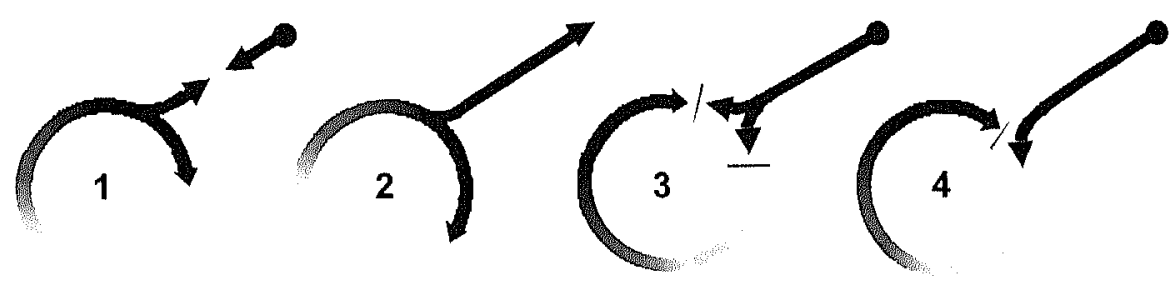

reentry circuit $=$ site of impulse formation

Figure 1.1 - The effect of timing, relative to the heart cycle, on the response of an extrastimulus.

The figure is modified from De Belder et al. ${ }^{38}$ Zone $1=$ collision zone; zone $Z$ = refractory zone; zone 3 = bidirectional block $/$ termination zone; zone $4=$ reset zone. For further explanation see paragraph 'Theoretical Background'. 
cuit (Figure 1.1: zone 2 = refractory zone). The wavefront initiated by a stimulus delivered in the tachycardia-termination window will terminate the tachycardia because it collides with the anterograde tachycardia wavefront and will not propagate itself because it meets the refractory tail of the impulse circulating in the tachycardia circuit (Figure 1.1: zone 3 = bi-directional block / termination zone). A late stimulus will also be able to enter the tachycardia circuit. Unlike the stimulus in the termination zone it will create unidirectional block only. It will block the anterograde wavefront of the tachycardia but will propagate in the circuit because the local tissue is no longer refractory and will reset the tachycardia (Figure 1.1: zone 4 = reset zone).

\section{Practical Considerations}

[14] These relatively simple theoretical circumstances are often difficult to accomplish in daily practice since many factors play a role in the ability to terminate reentrant tachycardias by pacing: ${ }^{21,33,39}$ (1) the tachycardia cycle-length, (2) the duration of the refractory period of the components of the reentry circuit, the tissue between the reentry circuit and the pacing site and the pacing site itself, (3) the distance between the tachycardia circuit (site of impulse formation) and the site of stimulation, (4) the conduction velocity in the circuit and in the tissue between the reentry circuil and the pacing site (Figure 1.2).

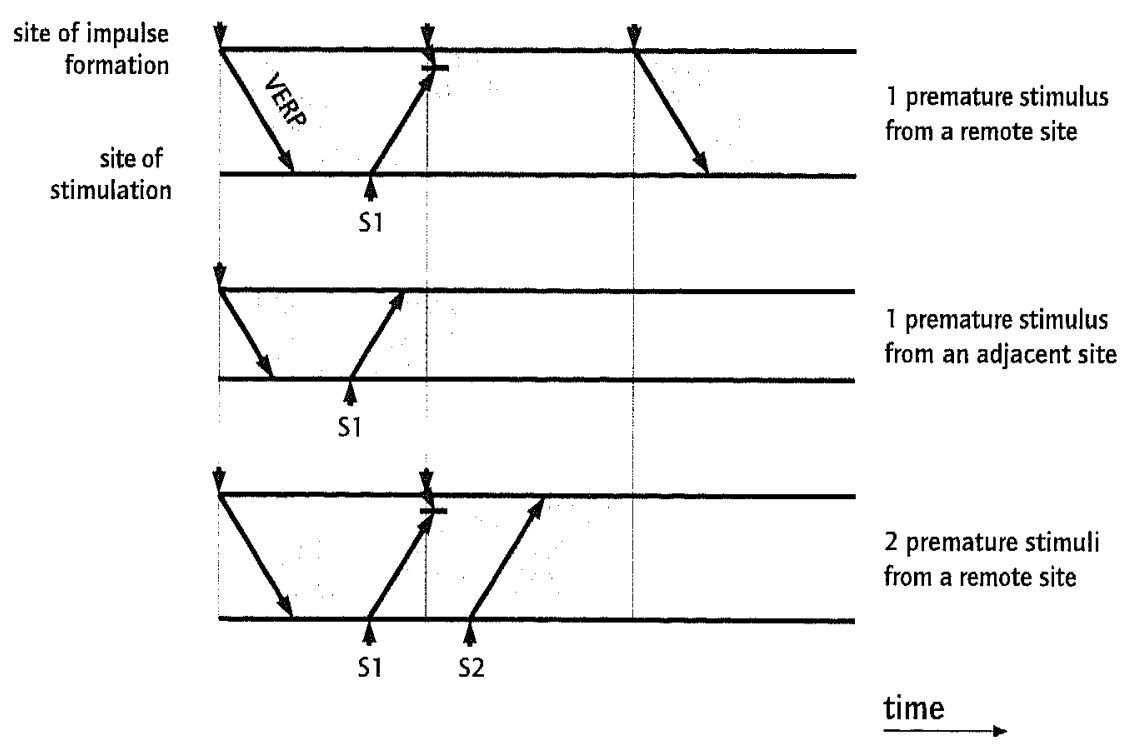

Figure 1.2 - Illustration of the critical parameters which determine entrance of premature stimuli into a reentry circuit.

The figure is modified from Fisher et al. ${ }^{43}$ For further explanation see paragraph 'Practical Considerations'. Abbreviation: $V E R P=$ ventricular effective refractory period. 
Therefore it is not unlikely that a single premature stimulus (S1) from a remote site can not reach the reentry circuit. The premature stimulus can not be delivered before the end of the refractory period. Before the stimulus can make its way back to the site of impulse formation the next tachycardia beat has already occurred (Figure 1.2, upper panel). If, under the same circumstances, a stimulus with the same coupling interval can be delivered from a site more adjacent to the reentry circuit, this stimulus can reach the site of impulse formation and can enter the reentry circuit (Figure 1.2, middle panel). Addition of a second premature stimulus (S2) facilitates entrance into the reentry circuit from a remote site of stimulation. The first premature stimulus (S1) will collide with the depolarization front coming from the reentry circuit, avoiding late depolarization of the site of stimulation. The second premature stimulus (S2) can be delivered immediately after the refractory period and will be able to reach the site of impulse formation and enter the reentry circuit (Figure 1.2, lower panel). By giving more than one premature stimulus, the refractory period of the tissue at the stimulation site and the intervening tissue can be further abbreviated or "peeled back", allowing delivery of earlier stimuli and earlier entrance into the reentry circuit. 40,41

The Role of the Implantable Cardioverter Defibrillator

An inherent risk of ATP is acceleration of the existing arrhythmia or initiation of a new arrhythmia. Therefore treatment of a VT with ATP can only be considered safe if backup defibrillation is present. With modern implantable cardioverter defibrillators (ICDs) both ATP and shock therapy can be provided.

Nevertheless, the use of ICD therapy to protect against sudden cardiac arrest is still limited. This is mainly due to the difficulty to identify the patient with a high risk of dying suddenly. In the Maastricht area in The Netherlands the yearly incidence of unexpected sudden cardiac arrest in a 4 year period (19911994) in a group between the ages of 25 and 75 years of 133,000 people was 1 per $1000 .{ }^{43}$ In Maastricht $5 \%$ of sudden cardiac arrest victims left the hospital neurologically intact after successful resuscitation. Assuming that this area is representative for The Netherlands this leads to an incidence of 1000 per million people per year with theoretically 50 survivors per million people per year. In 1996 only six ICD implants per million population in The Netherlands were reported ${ }^{4+4}$ with an expected increase to 25 ICDs in 1999. Although the yearly incidence of sudden cardiac arrest seems to be decreasing ${ }^{43}$ this still means that only $50 \%$ of the sudden cardiac arrest survivors have been instrumented with an ICD in The Netherlands in 1999. In Belgium and Germany the ICD implant rate in 1996 was three and four times that of The Netherlands. The highest implant rate of 89 ICDs per million population in 1996 was achieved in the USA. ${ }^{4+}$

Related to these numbers a few remarks have to be made. A lot of work has to be done to identify the patient with a high risk of dying suddenly before the first potentially lethal arrhythmia occurs. This will lead to an increase in primary indications for ICD implantation. Nevertheless, it is difficult to predict what 
Therefore it is not unlikely that a single premature stimulus (S1) from a remote site can not reach the reentry circuit. The premature stimulus can not be delivered before the end of the refractory period. Before the stimulus can make its way back to the site of impulse formation the next tachycardia beat has already occurred (Figure 1.2, upper panel). If, under the same circumstances, a stimulus with the same coupling interval can be delivered from a site more adjacent to the reentry circuit, this stimulus can reach the site of impulse formation and can enter the reentry circuit (Figure 1.2, middle panel). Addition of a second premature stimulus (S2) facilitates entrance into the reentry circuit from a remote site of stimulation. The first premature stimulus (S1) will collide with the depolarization front coming from the reentry circuit, avoiding late depolarization of the site of stimulation. The second premature stimulus (S2) can be delivered immediately after the refractory period and will be able to reach the site of impulse formation and enter the reentry circuit (Figure 1.2, lower panel). By giving more than one premature stimulus, the refractory period of the tissue at the stimulation site and the intervening tissue can be further abbreviated or "peeled back", allowing delivery of earlier stimuli and earlier entrance into the reentry circuit. 40,41

The Role of the Implantable Cardioverter Defibrillator

An inherent risk of ATP is acceleration of the existing arrhythmia or initiation of a new arrhythmia. Therefore treatment of a VT with ATP can only be considered safe if backup defibrillation is present. With modem implantable cardioverter defibrillators (ICDs) both ATP and shock therapy can be provided.

Nevertheless, the use of ICD therapy to protect against sudden cardiac arrest is still limited. This is mainly due to the difficulty to identify the patient with a high risk of dying suddenly. In the Maastricht area in The Netherlands the yearly incidence of unexpected sudden cardiac arrest in a 4 year period (19911994) in a group between the ages of 25 and 75 years of 133,000 people was I per $1000 .{ }^{43}$ In Maastricht $5 \%$ of sudden cardiac arrest victims left the hospital neurologically intact after successful resuscitation. Assuming that this area is representative for The Netherlands this leads to an incidence of 1000 per million people per year with theoretically 50 survivors per million people per year. In 1996 only six ICD implants per million population in The Netherlands were reported ${ }^{44}$ with an expected increase to 25 ICDs in 1999. Although the yearly incidence of sudden cardiac arrest seems to be decreasing ${ }^{4.3}$ this still means that only $50 \%$ of the sudden cardiac arrest survivors have been instrumented with an ICD in the Netherlands in 1999. In Belgium and Germany the ICD implant rate in 1996 was three and four times that of The Netherlands. The highest implant rate of 89 ICDs per million population in 1996 was achieved in the USA. ${ }^{\text {t4 }}$

Related to these numbers a few remarks have to be made. A lot of work has to be done to identify the patient with a high risk of dying suddenly before the first potentially lethal arrhythmia occurs. This will lead to an increase in primary indications for ICD implantation. Nevertheless, it is difficult to predict what 
impact the move to primary prevention will have on ICD use. Several studies, finished or still ongoing, suggest that this is currently a small percentage of patients known with heart disease (MADIT, ${ }^{45}$ AVID, ${ }^{46}$ MUSTT, ${ }^{47}$ DINAMIT ${ }^{48}$ ). Furthermore, it is interesting that the ICD implant rate for secondary prevention, which is still the most important indication for an ICD, differs so much between countries with rather similar levels of health care.

\section{Antitachycardia Pacing Modes}

In modern ICDs, ATP is the therapy of choice to start VT treatment. Compared to high-voltage shock therapy which could be used instead, ATP has two important advantages: it is not painful or anxiety provoking and it consumes far less battery power increasing the longevity of the ICD. Studies report success rates of ATP in VT between $70-90 \%$, with acceleration rates between $2-7 \%, 49-51$.

Modes of ATP can be divided in single and multiple beat modes. Single-beat modes are designed to deliver a single capturing stimulus to stop the tachycardia (Table 1.2). A limitation of these modes is that the site of stimulation has to be close to the reentry circuit to ensure penetration of the stimulus in the circuit (Figure 1.2). Since the position of an endocardial ICD lead is in the right ventricle and the reentry circuit is often located in the left ventricle, single beat ATP is rarely applied in ICDs. Multiple beat modes are designed to deliver two or more stimuli that depolarize or capture the heart without spontaneous activity in between (Table 1.3).

Although Table 1.2 and 1.3 contain the most commonly applied single- and multiple-beat ATP modes the variety in ATP modes is almost endless. Other

Table 1.2 single beat ATP modes.

underdrive
pacing
56,57

Abbreviations: $A T P=$ antitachycardia pacing; $R=$ sense moment of $R$-wave; $S I=$ premature stimulus, $R R=$ cycle length of the tachycardia. 
Table 1.3 Multiple beat ATP modes.

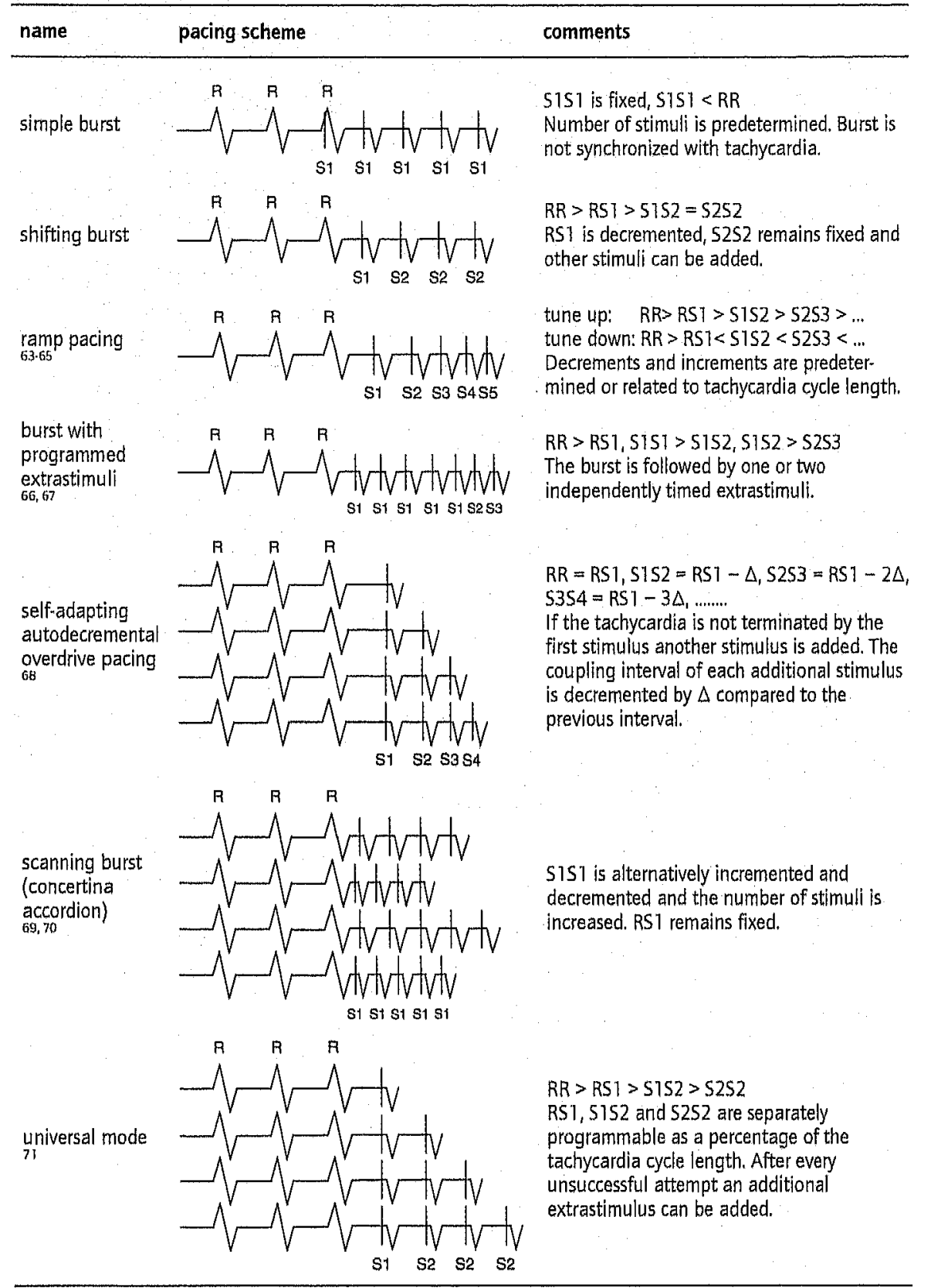

Abbreviations: ATP $=$ antitachycardia pacing; $R=$ sense moment of $R$-wave; $S 1,52,53, S 4, S 5=$ premature stimuli, RR = cycle length of the tachycardia. 
modes that can be found in the literature are: changing ramps, ${ }^{52}$ scanning burst with ramp pacing, programmed extrastimulation, extrastimulus $\operatorname{scan},,^{33}$ centrifugal scan, ${ }^{54,55}$ and self-searching scan. ${ }^{54,55}$

\section{Limitations of Current ATP Modes}

The aim of ATP is to pace in the termination window shortly after the refractory period (Figure 1.1). However, tachycardia rate, autonomic tone, antiarrhythmic drug level, ischemia, and other unknown factors influence the duration of the refractory period. Similar tachycardia rates do not necessarily have similar durations of refractoriness and for the same tachycardia rate, refractory periods will change over time. $+2,72$ Since these ATP modes do not 'know' the duration of the refractory period and none of these pacing modes adapts to changes in refrac-

[18 ] tory period not related to the tachycardia rate, the premature stimuli in the ATP burst scan over a certain range of coupling intervals. Since the peak of the endocardially-evoked I-wave coincides with the end of the refractory period it has been suggested that T-wave sensing may help in ATP.73 Sensing of the evoked T-wave may identify the end of the refractory period and hence guide appropriate timing of stimuli during ATP.74-76

\section{Aim of the Thesis}

The aim of this thesis is to study the relation between the stimulus-T interval (STI) and the duration of repolarization and refractoriness under the circumstances during which ATP will be applied. STI is the interval which starts at the moment of stimulation and ends at the moment of T-wave sensing in the downgoing slope of the T-wave of the endocardially-evoked response (Figure 1.3). Furthermore, if the duration of refractoriness can reliably be predicted by STI, a new ATP mode can be developed in which coupling intervals of premature stimuli are guided by STI information on a beat-to-beat basis. Finally the feasibility of this STI-based ATP mode will be tested.

\section{T-Wave Sensing}

Since reliable T-wave sensing is crucial for this ATP mode the background of T-wave sensing will be discussed. The ability to reproducibly sense the T-wave in an implantable pacing system was first described by Rickards et al. ${ }^{77}$ They showed that a capturing cathodal pacing pulse will evoke an endocardial response with a negative QRS complex followed by a positive T-wave (Figure 1.3). However, without special accommodations, polarization voltage will obscure the evoked response. Magnitude and time course of this polarization voltage depends on the energy delivered, the materials employed as cathode and anode, and the surface area of contact between electrodes and tissue. The polarity is opposite to the pacing stimulus with a magnitude of hundreds of millivolts decaying over a period of 


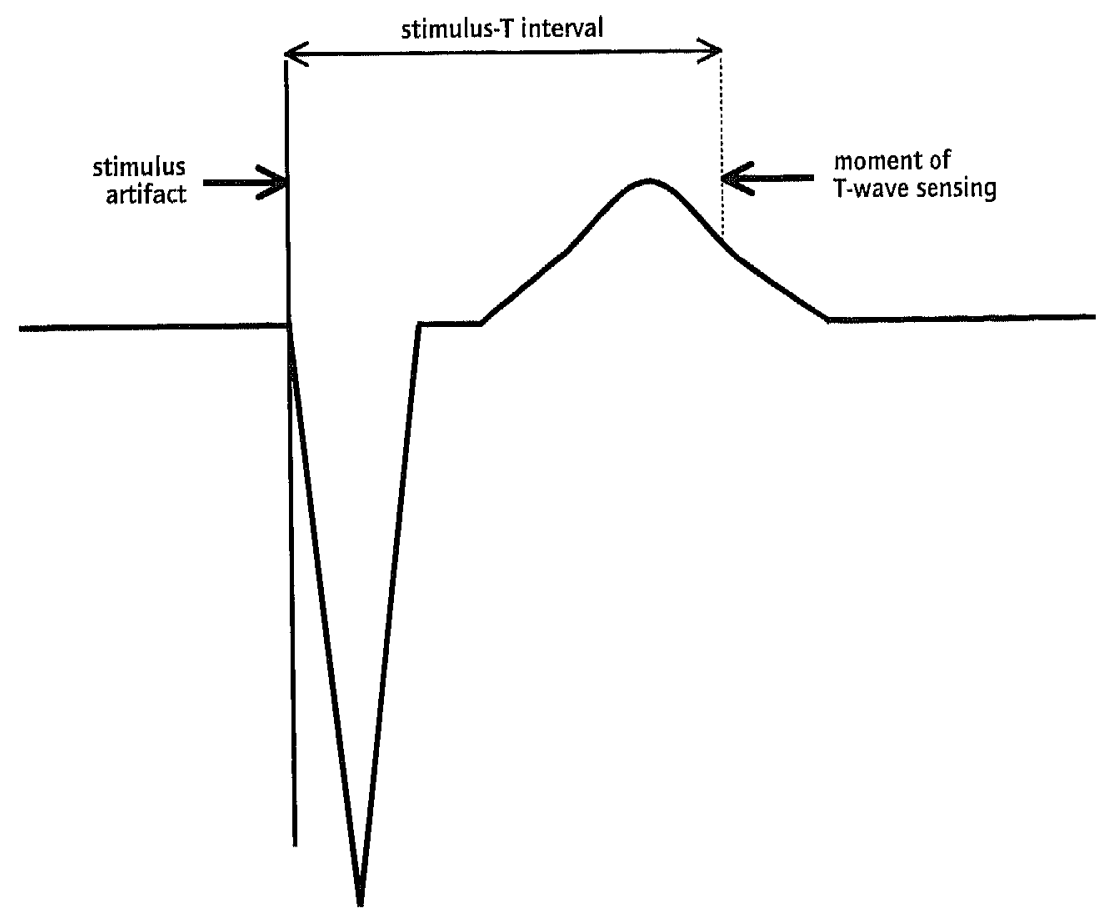

Figure 1.3 - Endocardially-evoked response after stimulation, with stimulus artifact, moment of T-wave sensing and stimulus-T interval indication.

more than $300 \mathrm{msec}$. Under these circumstances sensing of the evoked T-wave with a magnitude often lower than 10 millivolts is impossible. Using a triphasic pulse waveform (Vitatron Medical B.V., Dieren, The Netherlands) with a low-polarazing electrode, polarization effects can be minimized and the evoked response can be sensed within $5 \mathrm{msec}$ of the pulse. Detection is realized by performing an adjustable detection on the inverted derivative of the intracardiac signal. The Twave detection is enabled in an adjustable window to focus on the falling edge of the evoked T-wave. This window is reset after every stimulus. Further refinement of this idea led to an implantable pacing system capable of sensing the evoked Twave. During the experiments STI measurements were standardized using a custom-build stimulator (Vitatron Medical B.V., Dieren, The Netherlands) that allowed monitoring and recording of the endocardially-evoked response. This stimulator generated a I-wave marker indicating the moment of T-wave sensing in the endocardially-evoked T-wave. The sensitivity of evoked T-wave sensing was determined by decreasing the sensitivity setting until disappearance of the T-wave marker indicated the moment of undersensing. The value for T-wave sensitivity was programmed to half the value at which undersensing occurred. 
Since its introduction, STI was used to automatically adapt the pacing rate of implantable pacemakers to the level of activity of the patient, 77 as an indicator for drug-induced changes in patients, ${ }^{78}$ an early indicator for subendocardial ischemia, ${ }^{79}$ a differentiator between VT and sinus tachycardia, ${ }^{80}$ and as a system for automatic capture recognition. ${ }^{81}$

Applicability of the Stimulus-T Interval for Antitachycardia Pacing

Since T-wave sensing can be used to identify the end of the refractory period, this potential could be used in an implantable ATP system in which coupling intervals of premature stimuli could be determined by feed-back of STI information. ${ }^{73-76}$

This idea was initially tested in a series of AV block dogs (Chapter 2). ${ }^{75}$ In

[20] these dogs we were able to demonstrate a high correlation between STI and the ventricular effective refractory period (VERP) in the dynamic situation after a simulated VT. Based on these findings an ATP mode in which coupling intervals of premature stimuli are based on real-time STI information seemed feasible. Theoretically, such an ATP mode is superior to existing ATP modes because coupling intervals of premature stimuli are adapted to the actual duration of refractoriness on a beat-to-beat basis irrespective of the cycle length of the tachycardia. Therefore, this ATP mode might also be successful in the dynamic situation immediately after the onset of VT when the adaptation of repolarization and refractoriness is an ongoing process.

\section{Methodological Considerations}

Reproducibility of Ventricular Effective Refractory Period Measurements

Studying the literature related to the dynamic behavior of VERP only a limited number of publications was available. $82-87$ Furthermore clata were difficult to compare and seemed not to be reproducible (Table 1.4). Therefore we developed and tested a protocol to optimize reproducibility of VERP measurements under steady-state and dynamic circumstances (Chapter 3).88

Using this protocol we found considerable variations not only between individuals but also within one individual tested at different timepoints, stressing the importance of on-line measurements (Chapter 4).89 Therefore when accurate information on the VERP is needed, other highly correlated and on-line available parameters such as STI, could be of value (Table 1.4).80

\section{Repolarization and Refractoriness}

In the dynamic situation after the onset of VT it is difficult and time consuming to determine the behavior of VERP with high precision. ${ }^{83}$ Furthermore changes in the duration of repolarization are not always expressed by changes in refractoriness. Therefore, we decided to add the registration of the monophasic action potential (MAP). Although MAP registrations require a special catheter, 
which is not stable chronically, ${ }^{90}$ the duration of repolarization can be derived from the action-potential duration (APD) without the need of a special pacing protocol (Table 1.4). The MAP catheter was placed close to the electrode used for STI registrations. Therefore, MAP and STl registrations can be made simultaneously and from an adjacent endocardial site in the right ventricle.

\section{Effect of Cycle Length and Anti-Arrhythmic Medication}

Although we found a consistent relation between STI and VERP ${ }^{75}$ under drug free non-ischemic circumstances, this relation might change under the circumstances during which STI-based ATP will be applied. Patients often take antiarrhythmic drugs which not only prolong the duration but also change the dynamics of repolarization and refractoriness. ${ }^{91,92}$ Furthermore, cycle-length changes induced by VT show a considerable variation. Therefore we studied the influence of multiple cycle-length changes and d-sotalol, which prolongs the duration of repolarization, on the steady-state and dynamic behavior of VERP, APD, and STI (Chapter 5). ${ }^{76}$ These experiments were performed in our AV block dog model, later other investigators performed very similar experiments in humans. ${ }^{91}$

Table 1.4 Comparison of advantages and limitations of VERP, APD and STI determination.

\begin{tabular}{llll} 
& VERP & APD & STI \\
\hline on-line data & no & yes & yes \\
special lead requirements & no & yes & yes / no \\
reproducibility & low & high & high \\
available in implantable pacing system & no & no & yes \\
special hardware requirements & no & yes & yes \\
chronically available & no & no & yes \\
pacing (protocol) required & yes & no & yes (no) \\
represents repolarization & yes / no & yes & yes \\
represents refractoriness & yes & yes / no & yes / no \\
interference with spontaneous activity & yes & no & yes \\
evaluation of dynamic situation after change in heart rate & yes $/$ no & yes & yes \\
transmural & no & no & yes \\
size of monitoring area & focal & local & regional \\
\hline
\end{tabular}

Abbreviations: $V E R P=$ ventricular effective refractory period; $A P D=$ action potential duration; $S T I=$ stimulus-T interval. 
Furthermore, the combination of high rates, ischemia and antiarrhythmic drugs might lead to post repolarization refractoriness: a situation when refractoriness outlasts repolarization. We studied the relation between VERP and APD in this setting (Chapter 6). ${ }^{93}$

VTs often originate in the LV in the setting of left-sided ischemia. Since the electrode that will be used for ATP is most often positioned in the RV apex it is important to know how the duration of repolarization as measured in the normoxic RV relates to the duration of repolarization in the ischemic LV. Not only during steady-state circumstances but also in the dynamic situation after the onset of VI (Chapter 7).94

\section{Application of the Stimulus-T Interval for Antitachycardia Pacing}

After validation of the relation between VERP, APD, and STI under many different circumstances, ${ }^{76,93,94}$ the feasibility of STI-based ATP was tested in patients. Initial tests were performed in patients with AV nodal reentrant tachycardia and later feasibility tests were extended to patients with VT in the setting of old myocardial infarction. The results showed that STI-based ATP is both safe and feasible in these groups of patients (Chapter 8). It was beyond the scope of this work to compare the efficacy of STI-based ATP with other ATP modes.

\section{References}

1. Lewis I, Lond MD, Wales DS, et al. Single and successive extrasystoles. Lancet 1909; 1:382385.

2. Scott RW. Observations on a case of ventricular tachycardia with retrograde conduction. Heart $1922 ; 9: 297$

3. Mark IC, Berlin I, Kayden HJ, et al. The action of procaine amide ( $N$-[2-diethylaminuethyl]-paminobenzamide) on ventricular arthythmias. J Pharmacol Exper Therap 1950; 98:21-22.

4. Kayden HJ, Brodie BB, Steele JM. Procain amide. A review. Circulation 1957; 15:11.8-126.

5. Hooker DR, Kouwenhoven WB, Langworthy OR. The effect of alternating electrical currents on the heart. Am J Physiol 1933; 103:444-554

6. Wiggers C.J. The physiologic basis for cardiac resuscitation from ventricular librillation - method Lor serial defibrillation. Am Heart J 1940; 20:413-422.

7. Beck CS, Pritchard WH, Feil HS. Ventricular fibrillation of long duration abolished by electric shock. JAMA 1947; 135:985-986.

8. Zoll PM, Linenthal AJ, Gibson W, et al. Termination of ventricular fibrillation in man by externally applied electric countershock. N Engl J Med 1956; 254:727-732.

9. Lown B, Amarasingham R, Neuman J. New method for terminating cardiac arrhylhnias. Use of synchronized capacitor discharge. JAMA 1962; 182:548-555

10. Lown B, Neuman J, Amarasingham R, el al. Comparison of alternating current with direct current electroshock across the closed chest. Am J Cardiol 1962; 10:223-233.

11. Lown B. Electrical reversion of cardiac arrhythmias. Br Heart J 1967; 29:469-489. 
12. Mirowski M, Reid PR, Mower MM, et al. Termination of malignant ventricular arrhythmias with an implanted autonatic defibrillator in human beings. N Engl J Med 1980; 303:322-324.

13. Zoll PM, Linenthal AJ, Zarsky LRN. Ventricular fibrillation. Treatment and prevention by external electric currents. N Engl J Med 1960; 3:105-112.

14. Schwedel JB, Furman S, Escher DJW. Use of an intracardiac pacemaker in the treatrnent of Stokes-Adams seizures. Progr Cardiovasc Dis 1960; 3:170-177.

15. Sowton E, Leatham A, Carson P. The suppression of arrhythmias by artificial pacemaking Lancet 1964; 2:1098-1100.

16. Lenègre J, Maurice P. De quelques résultats obtenus par la dérivation direct intracavitaire des courants électriques de l'oreillette et du ventricule droits. Arch Mal Coeur 1945; 38:298-302.

17. Giraud G, Latour H, Puech P. Lactivité du noeud de Tawara el du Caisceau de His en electrocardiographie endocavitaire chez l'homne. Malattie Cardiovascolari 1960; 1:321-336.

18. Scherlag BJ, Lau SH, Helfant RH, et al. Catheter technique for recording His bundle activity in man. Circulation 1969; 39:13-18

19. Coumel P, Cabrol C, Fabiato A, et al. Tachycardie permanente par rythme réciproque. Arch Mal Coeur 1967; 60:1830-1864.

20. Durrer D, Schoo L, Schuilenburg RM, et al. The role of premature beats in the initiation and the termination of supraventricular tachycardia in the Wolff-Parkinson-White syndrome. Circulation 1967; 36:644-662.

21. Wellens HJ, Schuilenburg RM, Durrer D. Electrical stimulation of the heart in patients with ventricular tachycardia. Circulation 1972; 46:216-226.

22. Couch OA. Cardiac aneurysm with ventricular tachycardia and subsequent excision of aneurysm. Circulation 1959;20;251-253.

23. Harken AH, Horowitz LN, Josephson ME. Comparison of standard aneurysmectomy and aneutysmectomy with directed endocardial resection for the treatment of recurrent sustained ventricular tachycardia. J Thorac Cardiovasc Surg 1980; 80:527-534.

24. Sami M, Chaitman BR, Bourassa MG, et al. Long term follow-up of aneurysmectomy for recurrent ventricular tachycardia or fibrillation. Am Heart J 1978; 96:303-308.

25. Fontaine G, Frank R, Guiraudon G, et al. Surgical treatment of resistant reentrant ventricular tachycardia by ventriculotomy: A new application of epicardial mapping. Circulation 1974; 50 (Suppl III):82.

26. Gallagher JJ, Sealy WC, Anderson RW, el al. Cryosurgical ablation of accessory atrioventricular connections: a method for cortection of the pre-excitation syndrome. Circulation 1977; 55:471479 .

27. Josephson ME, Harken AH, Horowilz LN. Endocardial excision: a new surgical technique for the treatment of recurrent ventricular tachycardia. Circulation 1979; 60:1430-1439.

28. Borggrefe $M$, Budde $T$, Podczeck A, et al. High frequency alternating cutrent ablation of an accessory pathway in humans. I Am Coll Cardiol 1987; 10:576-582.

29. Soulhworth JL., McKusick VA, Pierce EC. Ventricular fibrillation precipitated by cardiac catheterization. JAMA 1950;143:717.

30. Hollman BF, Rosen MR. Cellular mechanisms for cardiac arrhythmias. Circ Res 1981; 49:1-15.

31. Wellens HJ, Lie KI, Durrer D. Further observations on ventricular tachycardia as studied by electrical stimulation of the heart. Chronic recurrent ventricular tachycardia and ventricular tachycardia during acute myocardial infarction. Circulation 1974; 49:647-653. 
32. Wellens HJ, Duren DR, Lie KI, Observations on mechanisms of ventricular tachycardia in man. Circulation 1976; 54:237-244

33. Wellens HJ. Value and limitations of programmed electrical stimulation of the heart in the study and treatment of tachycardias. Circulation 1978; 57:845-853.

34. Michelson EL, Spear JF; Moore EN. Electrophysiologic and anatomic correlates of sustained ventricular tachyarthythmias in a model of chronic myocardial infarction. Am J Cardiol 1980; 45:583-590

35. Wit AL, Allessie MA, Bonke FI, et al. Electrophysiologic mapping to determine the mechanism of experimental ventricular tachycardia initiated by premature impulses. Experimental approach and initial results demonstrating reentrant excitation. Am J Cardiol 1982; 49:166185 ,

36. Mines GR. On dynamic equilibrium in the heart. J Physiol 1913; 46:349-383.

37. Allessie MA, Bonke FI, Schopman FJ. Circus movement in rabbit atrial muscle as a mechanism of tachycardia. III. The "leading circle" concept: a new model of circus movement in cardiac tissue without the involvement of an anatomical obstacle. Circ Res 1977; 41:9-18.

38. De Belder MA, Malik M, Ward DE, et al. Pacing modalities for tachycardia termination. PACE $1990 ; 13: 231-248$.

39. Wellens HJ, Bär FW, Gorgels AP, et al. Electrical management of arrhythmias with emphasis on the tachycardias. Am J Cardiol 1978; 41:1025-1034.

40. Moe GK, Cohen W, Vick RL. Experimentally induced paroxysmal A-V nodal tachycardia in the dog. Am Heart J 1963; 65:87-92.

41. Moe GK, Mendez C. The physiologic basis of reciprocal thythm. Prog Cardiovasc Dis 1966; $8: 461-482$

42. Fisher JD, Kim SG, Matos JA, et al. Comparative effectiveness of pacing techniques for termination of well-tolerated sustained ventricular lachycardia. PACE 1983; 6:915-922.

43. de Vreede-Swagemakers JJ, Gorgels AP, Dubois-Arbouw WI, el al. Out-of-hospital cardiac arrest in the 1990's: a population-based study in the Maastricht area on incidence, characteristics and survival. J Am Coll Cardiol 1997; 30:1500-1505.

44. Garratt CJ. A new evidence base for implantable defibrillator therapy. Eur Heart J 1998; 19:189191

45. Moss AJ, Hall WJ, Cannom DS, et al. Improved survival with an implanted defibrillator in patients with coronary disease at high risk for ventricular arrhythmia. Multicenter Automatic Defibrillator Implantation Trial Investigators. N Engl J Med 1996; 335:1933-1940

46. A comparison of antiarthythmic-drug therapy with implantable defibrillators in patients resuscitated from near-fatal ventricular arrhythmias. The Antiarhythmics versus Implantable Defibrillators (AVID) Investigators. N Engl J Med 1997; 337:1576-1583.

47. Buxton AE, Lee KL, DiCarlo L, et al. Nonsustained ventricular tachycardia in coronary artery disease: relation to inducible sustained ventricular lachycardia. MUSTT Investigators. Ann Intern Med 1996; 125:35-39.

48. Nisam S, Mower M. KCD trials: an extraordinary means of determining patient risk? PACE 1998; $21: 1341-1346$

49. Kantoch MJ, Green MS, Tang AS. Randomized cross-over evaluation of two adaptive pacing algorithms for the termination of ventricular tachycardia. PACE 1993; 16:1664-1672. 
50. Trappe HJ, Klein H, Kielblock B. Role of antitachycardia pacing in patients with third generation cardioverter defibrillators. PACE 1994; 17:506-513.

51. Newman D, Dorian P, Hardy J. Randomized controlled comparison of antitachycardia pacing algorithms for termination of ventricular tachycardia. J Am Coll Cardiol 1993; 21:1413-1418.

52. Furman S. Therapeutic uses of atrial pacing. Am Heart J 1973; 86:835-840.

53. Spurrell RA, Nathan AW, Bexton RS, et al. Implantable automatic scanning pacemaker for termination of supraventricular tachycardia. Am J Cardiol 1982; 49:753-760.

54. Sowton E. Clinical resulss with the Tachylog antitachycardia pacemaker. PACE 1984; 7:13131317.

55. Holt P, Crick JC, Sowton E. Antitachycardia pacing: a comparison of burst overdrive, selfsearching and adaptive table scanning programs. PACE 1986; 9:490-497.

56. Ryan GF, Easley RM, Zaroff Ll, et al. Paradoxical use of a demand pacemaker in treatment of supraventricular tachycardia due to the Wolff-Parkinson-White syndrome. Observation on termination of reciprocal rhythm. Circulation 1968; 38:1037-1043.

57. den Dulk K, Lindemans FW, Bär FW, et al. Pacemaker related tachycardias. PACE 1982; 5:476485.

58. Critelli G, Grassi G, Chiariello M, et al. Automatic "scanning" by radiofrequency in the longterm electrical treatment of arrhythmias. PACE 1979; 2:289-296

59. Nathan A, Hellestrand K, Bexton R, el al. Clinical evaluation of an adaptive tachycardia intervention pacemaker with automatic cycle-length adjustment. PACE 1982; 5:201-207.

60. Waxman HL, Cain ME, Greenspan AM, et al. Termination of ventricular tachycardia with ventricular stimulation: salutary effect of increased current strength. Circulation 1982; 65:800-804.

61. Spurrell RA, Sowton E. Pacing techniques in the management of supraventricular tachycardias. Parl 1. The use of high frequency stimulation (HFS) in the management of paroxysmal supraventricular tachycardia. J Electrocardiol 1975; 8:287-295.

62. Fisher JD, Ostrow E, Kim SG, et al. Ultrarapid single-capture train stimulation for termination of ventricular tachycardia. Am J Cardiol 1983; 51:1334-1338.

63. Nathan AW, Camm AJ, Bexton RS, et al. Initial experience with a fully implantable, programmable, scanning, extrastimulus pacemaker for tachycardia termination. Clin Cardiol 1982; 5:22-26.

64. Ward DE, Camm AJ, Gainsborough J, et al. Autodecremental pacing -a microprocessor based modality for the termination of paroxysmal tachycardias. PACE 1980; 3:178-191.

65. den Dulk $K$, Bertholet $M$, Brugada $P$, et al. A versatile pacemaker system for termination of tachycardias. Am J Cardiol 1983; 52:731-738.

66. Gardner M], Waxman HL, Buxton AE, et al. Termination of ventricular tachycardia. Evaluation of a new pacing method. Am J Cardiol 1982; 50:1338-1345.

67. Jentzer JH, Hoffmann RM. Acceleration of ventricular tachycardia by rapid overdrive pacing combined with extrastimuli. PACE 1984; 7:922-924.

68. Charos GS, Haffajee CI, Gold RL, et al. A theoretically and practically more effective method for interruption of ventricular tachycardia: self-adapting autodecremental overdrive pacing. Circulation 1986; 73:309-315.

69. Nathan A, Hellestrand K, Ward D, et al. Rate-related accelerating (autodecremental) atrial pacing for reversion of paroxysmal supraventricular tachycardia. J Electrocardiol 1982; 15:77-84. 
70. Spurrell RA, Nathan AW, Camm AJ. Clinical experience with implantable scanning tachycardia reversion pacemakers. PACE 1984; 7:1296-1300.

71. den Dulk K, Kersschot IE, Brugada $P$, el al. Is there a universal antitachycardia pacing mode? Am J Cardiol 1986; 57:950-955.

72. Kappenberger L, Sowton E. Programmed stimulation for long-term treatment and non-invasive investigation of recurrent tachycardia. Lancet 1981; 1:909-914

73. Begemann M, Boute W, Wittkampl FH. Evoked endocardial potentials in tachycardia management. (abstract) PACE 1987; 10:608.

74. Begemamn MJS, Boute W. Automatic refractory period. PACE 1988; 11:1684-1686.

75. den Dulk K, Leerssen $H$, Vos $M$, et al. Applicability of the stimulus-I interval for antitachycardia pacing. PACE 1991; 14(Pt. Il):1757-1761.

76. Leerssen HM, Vos MA, den Dulk K, et al. Steady-state and dynamic behavior of ventricular repolarization and refractoriness in the dog: the effect of multiple cycle-length changes and d-sotalol administration. PACE 1998; 21:1766-1777.

77. Rickards AF, Norman J. Relation between QT interval and heart rate. New design of physiologically adaptive cardiac pacemaker. Br Heart J 1981; 45:56-61.

78. Donaldson RM, Rickards AF. Evaluation of drug-induced changes in myocardial repolarisation using the paced evoked response. Br Heart J 1982; 48:381-387.

79. Donaldson RM, Taggart P, Swanton H, et al. Intracardiac electrode detection of early ischaemia in man. Br Heart J 1983; 50:213-221.

80. Belz MK, Ellenbogen KA, Camm AJ, et al. Diflerentiation between monomorphic ventricular tachycardia and sinus tachycardia based on the right ventricular evoked potential. PACE 1992; 15:1661-1666.

81. Bolz A, Hardt R, Hubmann M, et al. A new autocapture logic based on fractally coated low polarization pacing leads. (abstract) PACE 1993; 16:1914.

82. Han J, Moe GK. Cumulative effects of cycle length on refractory periods in cardiac tissues. Am J Physiol 1969; 217:106-109.

83. Janse MJ, van der Steen $A B$, van Dam RT, et al. Refractory period of the dog's ventricular myocardium following sudden changes in frequency. Circ Res 1969; 24:251-262.

84. Brownstein SL, Blackwell WH, Welch WJ, et al. Cumulative effects of cycle length on ventricular refractoriness in man. Am Heart J 1990; 119:324-330.

85. Wiener I, Kunkes S, Rubin D, et al. Effects of sudden change in cycle length on human atrial, atrioventricular nodal and ventricular refractory periods. Circulation 1981; 64:245-248.

86. Morady F, Kadish AH, Toivonen LK, et al. The naximum effect of an increase in rate on human ventricular refractoriness. PACE 1988; 11:2223-2234.

87. Rosenheck S, Schmaltz S, Kadish AH, et al. The effect of quinidine and mexiletine on the adaptation of ventricular refractoriness to an increase in rate. Am Heart J 1991; 121:512-517.

88. Leerssen HM, Vos MA, den Dulk K, et al. Is the ventricular effective refractory period different when determined by incremental versus decremental scanning?: the effect of pacing cycle length, d-sotalol, and levcromakalim. PACE 1994; 17(Pt. Il):2084-2089.

89. Leerssen $H M$, Vos $M A$, den Dulk $K$, et al. Inter- and intraindividual variations in shortening of ventricular effective refractory period after an abrupt decrease in pacing cycle lengh. PACE 1994; 17(Pt. II):2079-2083. 
90. Franz MR, Swerdlow CD, Liem LB, et al. Cycle-length dependence of human action-potential duration in-vivo. Effects of single extrastimuli, sudden sustained rate acceleration and deceleration, and different sleady-state frequencies. J Clin Invest 1988; 82:972-979.

91. Lee $S D$, Dorian P. Geist M, et al Validation of a noninvasive measure of local myocardial repolarization in a conscious human model: adaptation of repolarization to changes in rate. J Cardiovasc Electrophysiol 1999; 10:1171-1179

92. Melichercik J, Brachmann J, Schols W, et al. Rate and time dependent effects of d-sotalol on the monophasic action potential after sudden increase of the heart rate. PACE 1999; 22:65-72.

93. Leerssen HM, Vos MA, den Dulk K, et al. Rate-dependent eflects of procainamide on the threshold current for pacing in the setting of postrepolarization refractoriness in dogs. PACE 1999 22:291-301

94. Leerssen HM, Vos MA, Houben R, et al. High uniformity of left and right ventricular repolarization dynamics induced by an abrupt decrease in pacing cycle length in a dog is not affected by left-ventricular ischemia. J Cardiovase Electrophysiol 2000: 11:42 I-429. 


\section{Chapter 2}

\section{Applicability of the Stimulus-T Interval for Antitachycardia Pacing}

Karel den Dulk, Hendrik M. Leerssen, Marc A. Vos, Willem R.M. Dassen, Jan Kersemakers, Malcolm J. Begemann, ${ }^{*}$ and Hein J.J. Wellens.

Department of Cardiology, Cardiovascular Research Institute Maastricht, Maastricht University, The Netherlands, and *Vitatron Medical B.V., Dieren, The Netherlands.

Published in PACE 1991; 14(Pt. II):1757-1761. 


\section{Abstract}

Introduction: At the onset of tachycardia, the refractory period (RP) changes together with the tachycardia termination window. We evaluated dogs with total atrioventricular (AV) block to determine if stimulus-T interval (STI) can be used to adjust the coupling interval(s) of an antitachycardia pacemaker in relation to changes in RP.

Methods and Results: Endocardial STI was recorded continuously together with six surface ECG leads. Steady-state ( $>2 \mathrm{~min}$ ) was determined for drive cycle lengths (DCL) 400 and 900 msec. The test pulse (TP) coupling interval, with DCL 900 msec, was chosen to be equal to the RP of DCL 400 msec. DCL was then changed to $400 \mathrm{msec}$ until TP captured. STI of the beat before capture was gained was

[30] measured. DCL was then changed back to $900 \mathrm{msec}$ and the interval determined when capture was lost. TP was then lengthened by $5 \mathrm{msec}$ and the procedure repeated until TP captured immediately upon changing to DCL $400 \mathrm{msec}$. The difference between RP at onset of pacing at DCL of $400 \mathrm{msec}$ and RP when capture was achieved with the shortest coupling interval was 35-50 (mean 40) msec. This required 35-90 (mean 62) seconds. The correlation coefficient RP to STI was $>0.95$.

Conclusion: (1) RP changed by as much as $35-50 \mathrm{msec}$ at the onset of an abrupt increase in rate in a 35-90 second period; and (2) STI enables estimation of RP on a beat-to-beat basis. Capture can therefore be predicted from the previous beat and the coupling interval adjusted accordingly in an antitachycardia pacing mode. 


\section{Introduction}

Termination of a reentrant tachycardia by pacing is influenced by tachycardia rate, refractory period (RP), autonomic tone, drug level, and other unknown factors. Several antitachycardia pacing modes are available to cope with a changing tachycardia termination window such as interval scanning, ${ }^{1}$ high frequency stimulation, ${ }^{2}$ an automatic increase in the number of stimuli, ${ }^{3}$ the adaptive mode, ${ }^{3}$ and the universal mode. ${ }^{4}$ None of these pacing modes adapts to changes in RP. The RP is longest at the onset of tachycardia. As demonstrated by Janse et al. $54 \%$ to $60 \%$ of the total shortening in the RP occurs in the first 20 beats. The final value is reached after 400 to 500 beats. Adaptation of the coupling interval to the local RP would be a further refinement of the universal antitachycardia pacing mode. In this study we evaluated if the stimulus-T interval (STI) can be used to adjust the coupling intervals of an antitachycardia pacing mode in accordance to changes in RP.

\section{Methods}

Preparation of Study Dogs

The experiments were performed in five mongrel dogs of either sex having a body weight between $20-35 \mathrm{~kg}$. All animals had a right thoracotomy followed by injection of formalin 37\% to induce atrioventricular (AV) block. "The animals were cared for according to standards set by the American Physiological Society. Experiments were not performed in the first 2 postoperative weeks because spontaneous episodes of ventricular tachycardia are known to occur after creation of AV block. ${ }^{7}$

\section{Experiments}

Anesthesia was induced with intravenous sodium pentobarbital ( $20 \mathrm{mg} / \mathrm{kg}$ ). The animals were intubated and ventilated with a mixture of oxygen, nitrous oxide, and halothane. Vitatron, model TP 53, unipolar endocardial stimulating electrodes (Vitatron Medical B.V., Dieren, The Netherlands) were introduced into the right ventricle via an extemal jugular vein using the cut-down technique. Adequate pacing and stimulating thresholds were required before securing the lead with the anchoring sleeve. The pacing leads were connected to a Vitatron investigational battery powered stimulator, which delivers pacing pulses with a dual fast recharge pulse form, enabling polarization-free recording directly from the stimulating electrode. This stimulator was triggered by a commercially available electrophysiology stimulator (Medtronic Model 5325 (Medtronic, Inc., Minneapolis, MN, USA)). The polarization-free recording enabled registration and analyses of the evoked T-wave. The endocardial signal from the pacing electrode was recorded simultaneously with six surface ECG leads at a paper speed of $100 \mathrm{~mm} / \mathrm{sec}$ as shown in Figure 2.1. Recordings were made continuously during the pacing protocol. 


\section{Pacing Protocol}

Steady-state RP ( $>2 \mathrm{~min}$ ) was determined for drive cycle lengths (DCL) 900 and $400 \mathrm{msec}$, respectively, by delivering a test stimulus at selected intervals every eighth beat without an intertrain pause. The interval between the test stimulus and the next drive stimulus was always equal to the DCL. Subsequently the coupling interval of the test stimulus was chosen to be equal to the steady-state RP at DCL $400 \mathrm{msec}$, while pacing at a DCL of $900 \mathrm{msec}$. The DCL was then changed to $400 \mathrm{msec}$ until the test stimulus captured the ventricle (first to capture) as shown in Figure 2.1. Time taken for the test stimulus to capture the ventricle when changing the DCL to $400 \mathrm{msec}$, as well as STI of the drive stimulus just preceding capture, were measured and recorded (Figure 2.1). The DCL was then changed back to $900 \mathrm{msec}$ and the interval determined when capture of the [32] test stimulus was lost. The STI of the drive stimulus preceding the last test stimulus to capture (last to capture) was measured and recorded. The coupling interval of the test pulse (TP) was then lengthened by $5 \mathrm{msec}$ and the procedure was repeated. This was done until the coupling interval of the test stimulus was such that immediate capture of the TP occurred (and not until the coupling interval equaled the steady-state RP at DCL $900 \mathrm{msec}$ ) when changing the DCL from 900 to $400 \mathrm{msec}$.

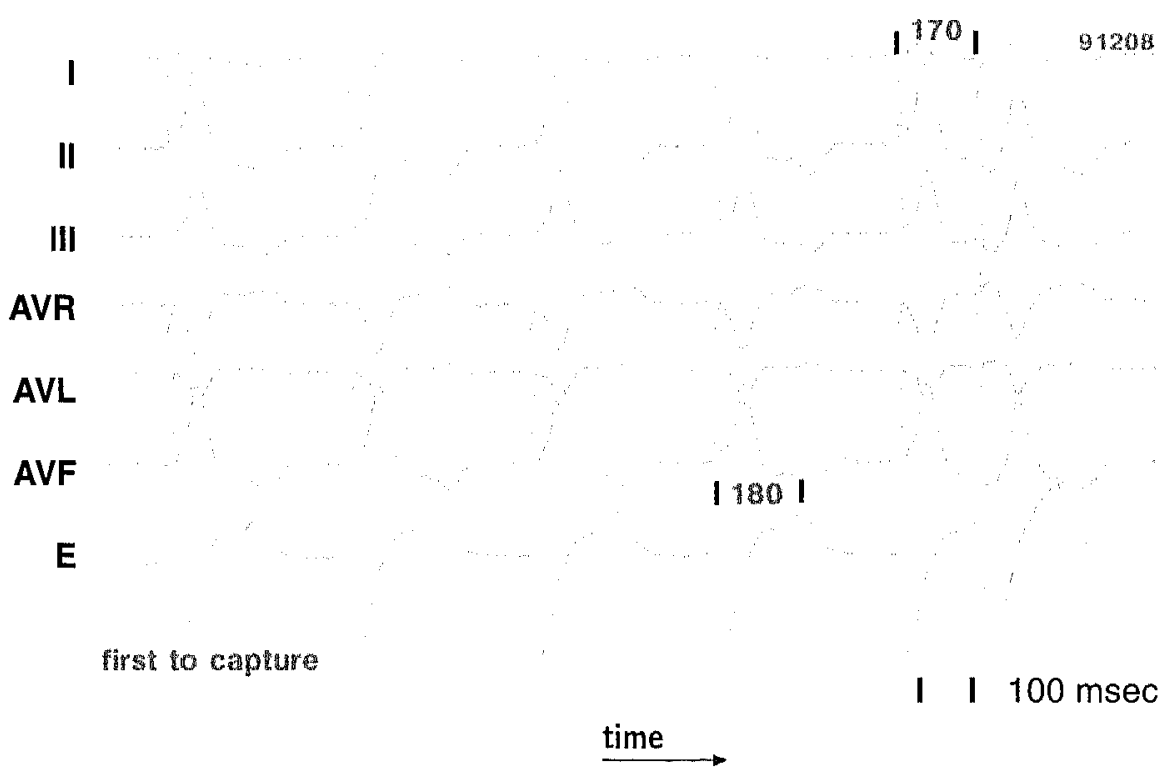

Figure 2.1 - Simultaneously recorded 6-lead electrocardiogram and endocardial evoked T-wave (E).

In this dog, the stimulus-T interval was $180 \mathrm{msec}$ when the test stimulus with coupling interval $170 \mathrm{msec}$ first captured. 


\section{Results}

\section{Correlation of Stimulus-T Interval Versus Refractory Period}

Figure 2.2 shows the comparison STI-RP for dog 4 when the DCL was changed from 900 to $400 \mathrm{msec}$ with the various coupling intervals (first to capture). As can be seen from the figure, there is a high correlation between STI and the RP. In all dogs the correlation coefficient between RP and STI was > 0.95 (both measurements, first and last to capture).

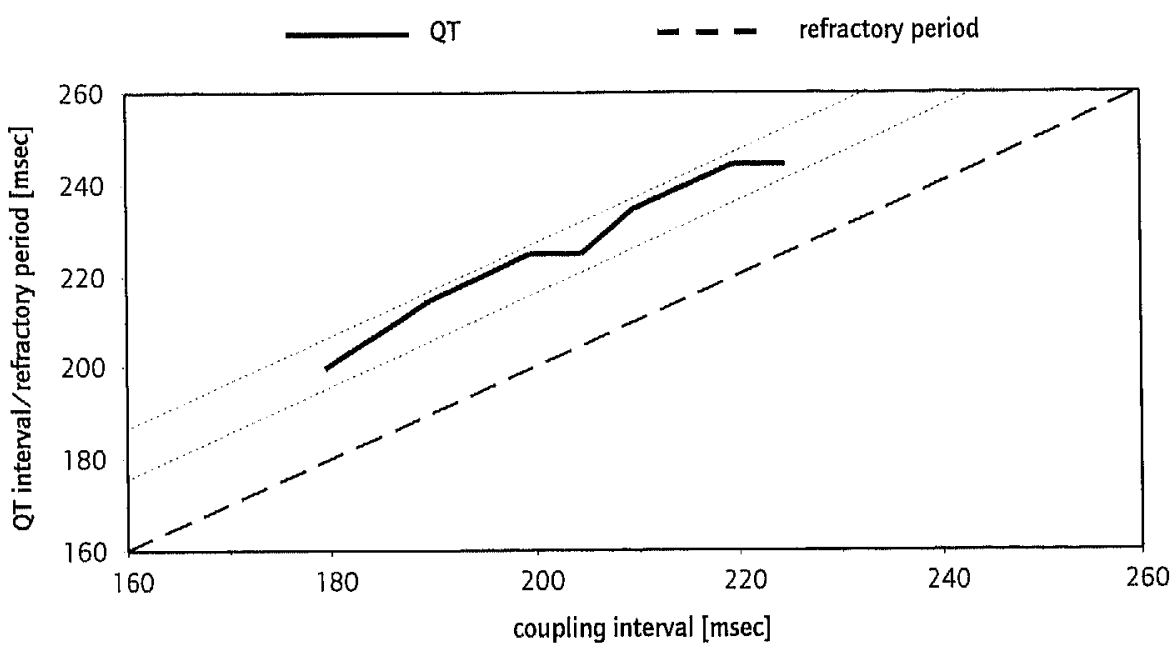

Figure 2.2 - Relation QT interval to refractory period.

Graph showing the high correlation between stimulus- $T$ and ventricular refractory period in dog 4 for first to capture coupling intervals 180 to $225 \mathrm{msec}$.

\section{Refractory Period}

The mean difference between the two steady-state RPs was $56 \mathrm{msec}$. The mean steady-state RP at DCL $900 \mathrm{msec}$ was $235 \mathrm{msec}$. To evaluate the degree of shortening of the RP after the initial shortening by the first few beats (relevant for antitachycardia pacing) when the DCL was changed from 900 to $400 \mathrm{msec}$, values for RP and STI were not used if capture was achieved with the first attempt. Using these criteria the RP shortened by $35-50 \mathrm{msec}$ (mean $40 \mathrm{msec}$ ).

\section{Stimulus-T Interval}

Figure 2.3 depicts the change in STI after changing the DCL from 900 to $400 \mathrm{msec}$ for each dog with the test stimulus with the shortest coupling interval (first to capture). The abscissa represents the number of beats at the onset of the 


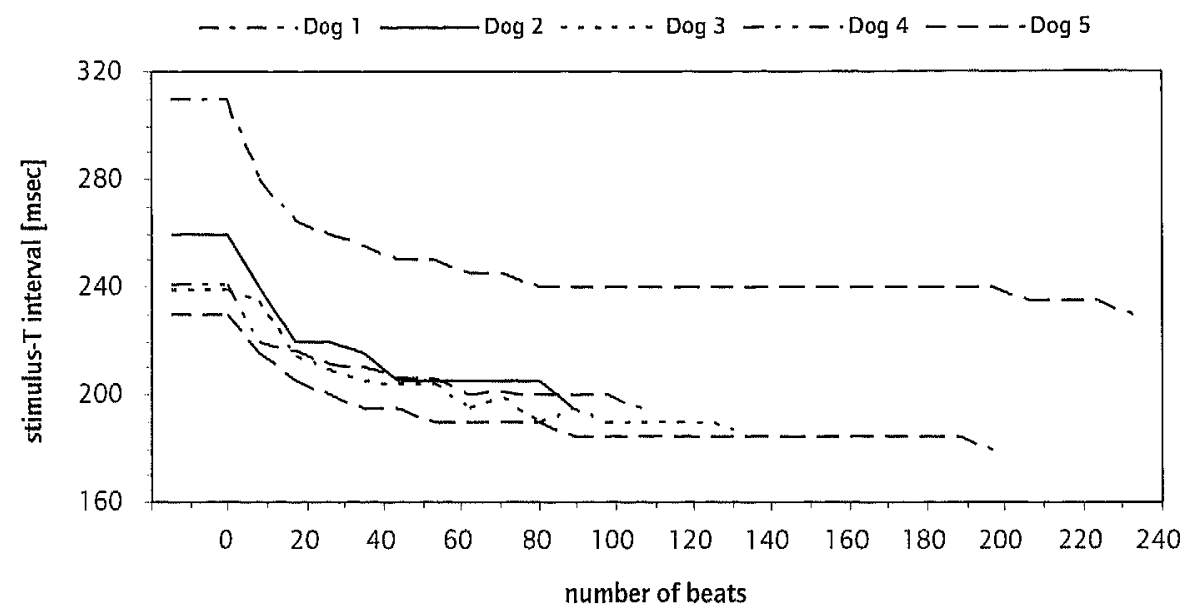

Figure 2.3 - Stimulus-T with abrupt change in drive cycle length from 900 to $400 \mathrm{msec}$. Graph depicting the change in stimulus-T interval when determining the first to capture interval for each dog with the test stimulus equal to the steady-state refractory period at drive cycle length $400 \mathrm{msec}$.

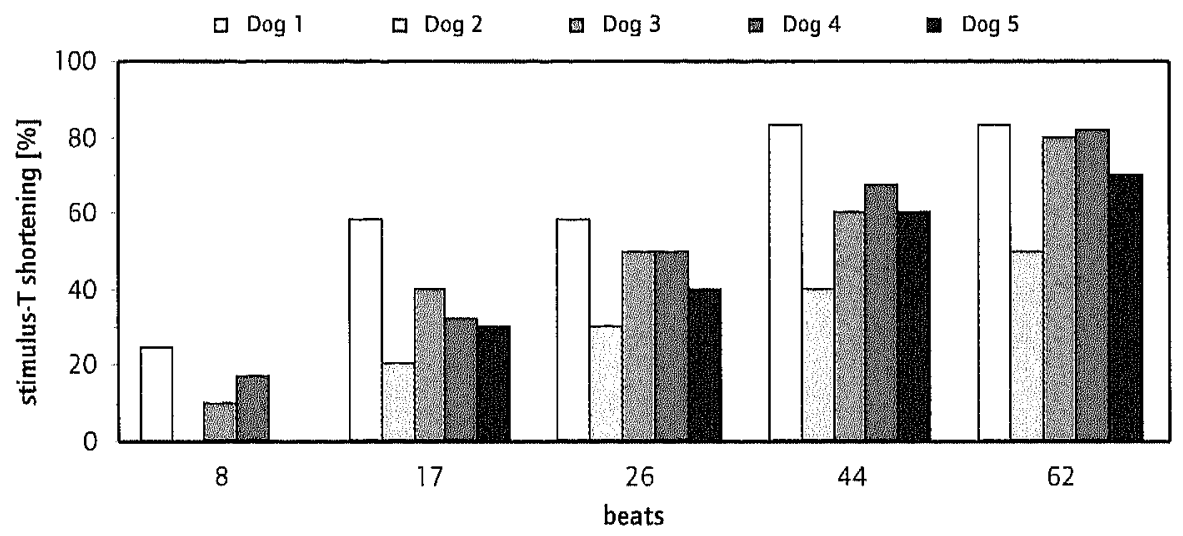

Figure 2.4 - Stimulus-T interval shortening at the onset of pacing at cycle length $400 \mathrm{msec}$. Bar chart indicating the percentage shortening of the stimulus-T interval from beat 2 at the onset of pacing with drive cycle length $400 \mathrm{msec}$ with a first to capture coupling interval equal to the steady-state refractory period of cycle length $400 \mathrm{msec}$.

drive cycle of $400 \mathrm{msec}$. Zero represents the last beat with coupling interval 900 msec. The end of each line indicates the point of first to capture, which took 35 to 90 seconds. The STI shortens by $9-38 \%$ (mean 24\%) with the first two beats. Figure 2.4 shows the percentage STI shortening from beat 2 to respectively beats 
$8,17,26,44$, and 62 . The mean STI shortening of beats $2-8$ was $9 \%$, beats $2-17$ was $36 \%$, beats $2-26$ was $46 \%$, beats $2-44$ was $62 \%$, and beats $2-62$ was $73 \%$. One hundred percent shortening is reached when capture is achieved.

\section{Discussion}

\section{Correlation Refractory Period Stimulus-T Interval}

There was a high correlation of STI to RP $\left(r^{2}>0.95\right)$. This was the case for first and last to capture in all dogs. This suggests that STI can be used reliably to predict capture of premature antitachycardia pacemaker stimuli in the ventricle.

Initial Shortening of Refractory Period

Ventricular RP was influenced by several factors such as rate (pacing, tachycardia), autonomic tone, and medication. The mean difference between the two steady-state RPs was $56 \mathrm{msec}$. Janse et al. ${ }^{5}$ demonstrated in three dogs that $27 \%$ of the total shortening occurred by the first beat of the faster rate and $40 \%$ to $45 \%$ by the second. After the first two beats the rate of change suddenly becomes slower and longer. Looking at the STI, we found a similar initial response in some of the dogs. STI shortened by $9-38 \%$ with the first two beats.

\section{Antitachycardia Pacing}

Figure 2.3 shows that there is still a significant amount of shortening of STI up to beat 44 , which occurs at a time when five to six attempts to terminate tachycardia with a pacemaker could have been tried. The mean shortening of the RP after the first few beats was 40 msec. The difference in RP could be enlarged by: a larger difference in cycle length; the onset of tachycardia may give rise to additional sympathetic stimulation, which could also have the effect of further shortening the RP making the difference greater; and there could be a significant difference in RP if tachycardia occurred during sleep as opposed to exercise or emotion. Similarly, antiarrhythmic drugs have an important influence on the ventricular RP and can be of importance for antitachycardia pacing, especially with changing drug levels or if medication is forgotten.

\section{Electrophysiology}

Morady et al. ${ }^{8}$ demonstrated that determination of the ventricular effective RP by using 8-beat drive trains and a 4 seconds intertrain pause may often overestimate the actual ventricular effective RP because a longer period of pacing is required to reach the steady-state RP. This can be corrected by recording the endocardial STI continuously and thus having a continuous reference, which could also be of value to understand the mechanism of various antiarrhythmic drugs during the electrophysiological study. 


\section{Conclusion}

STI that can be accurately detected and measured by an implantable device enables the implementation of an antitachycardia pacing mode which can adapt to important changes in RP. This should enable quicker, safer, and more reliable termination of tachycardia from the ventricle. It remains to be seen if this will have an influence on the incidence of acceleration during pacing for ventricular tachycardia. Simultaneous STI measurements during routine electrophysiological studies could be used as a continuous reference on ventricular refractoriness and could assist in the understanding of arrhythmia mechanisms.

\section{References}

1. Spurrell RA, Nathan AW, Bexton RS, et al. Implantable automatic scanning pacemaker for termination of supraventricular tachycarclia. Am J Cardiol 1982; 49:753-760.

2. Spurrell RA, Sowton E. Pacing techniques in the management of paroxysmal supraventricular tachycardia. The use of high frequency stimulation (HFS) in the management of paroxysmal supraventricular tachycardia. J Electrocardiol 1975; 8:287-295.

3. den Dulk $K$, Bertholeı $M$, Brugada P, et al. A versatile pacemaker system for termination of lachycardias. Am J Cardiol 1983; 52:731-738

4. den Dulk K, Kersschot IE, Brugada $P$, et al. Is there a universal antitachycardia pacing mode? Am.J Cardiol 1986; 57:950-955.

5. Janse $M J$, van der Steen $A B$, van Dam RT, et al. Refractory period of the dog's ventricular myocardium following sudden changes in frequency. Circ Res 1969; 24:251-262.

6. Steiner C, Kovalik TW. A simple technique for production of chronic complete heart block in dogs. J Appl Physiol 1968; 25:631-632.

7. Vassalle $M$, Knob RE, Cummins $M$, et al An analysis of fast idioventricular rhythm in the dog Circ Res 1977; 41:218-226

8. Morady $\mathrm{F}$, Kadish AH, Toivonen LK, et al. The maximum effect of an increase in rate on human ventricular refractoriness. PACE 1988; 11:2223-2234. 


\section{Chapter 3}

\section{Is the Ventricular Effective Refractory Period \\ Different when Determined by Incremental Versus}

Decremental Scanning?: The Effect of Pacing Cycle

Length, d-Sotalol and Levcromakalim

Hendrik M. Leerssen, Marc A. Vos, Karel den Dulk, Jolanda'van der Zande, and Hein J.J. Wellens.

Department of Cardiology, Cardiovascular Research Institute Maastricht, Maastricht University, The Netherlands.

Published in PACE 1994; 17(Pt. II):2084-2089. 


\section{Abstract}

Introduction: In the clinical setting the ventricular effective refractory period (VERP) is determined by an eight beat drive train S1S1, followed by a premature stimulus $\$ 2$, which is decremented in subsequent drive trains until capture is lost. Variation in intertrain pauses and capturing extra stimuli will disturb steady-state circumstances and reduce reproducibility of values found for VERP. To increase reproducibility, a protocol without intertrain pause and incremental scanning (IS) of $\$ 2$ was developed.

Methods and Results: In anesthetized dogs with chronic atrioventricular (AV) block, determination of the VERP using IS and decremental scanning (DS) without intertrain pause was compared at 800 and $350 \mathrm{msec}$ pacing cycle length

[38] (PCL). The measurements were repeated after the administration of d-sotalol to lengthen the VERP and levcromakalim to shorten the VERP. The results showed no difference between IS and DS at both PCLs with or without medication. Recurrent and abrupt rate changes were avoided during IS.

Conclusion: No differences in VERP were found comparing IS to DS at two PCLs with or without medications that affect the VERP. 


\section{Introduction}

The pacing protocol most commonly used to determine the ventricular effective refractory period (VERP) in the human electrophysiology laboratory consists of a drive train of eight basic cycles (S1), with an interstimulus interval ranging between $400-600 \mathrm{msec}$. This drive train is followed by a premature stimulus S2 which scans diastole. The initial coupling interval S1S2 is chosen to be longer than the VERP. In subsequent drive trains, S1S2 is progressively shortened until capture is lost. ${ }^{1-3}$ Due to the pause between subsequent drive trains and the capturing S2, steady-state conditions will continuously be disturbed.

Recently, the incremental scanning (IS) technique was introduced, which uses an incrementing \$1S2, initially shorter than the VERP., ${ }^{45}$ Using this protocol Morady et al. ${ }^{4}$ reported no difference in outcome comparing IS and decremental scanning (DS), whereas Beauregard et al. ${ }^{5}$ found longer values for the VERP using IS compared to DS.

We extended these observations, comparing IS versus DS after the administration of $\mathrm{d}_{\text {-sotalol }}{ }^{6}$ to lengthen the VERP and levcromakalim ${ }^{7}$ to shorten the VERP at 800 and $350 \mathrm{msec}$ pacing cycle length (PCL) in anesthetized dogs with chronic atrioventricular (AV) block.

\section{Methods}

This protocol was approved by the local ethical committee for experiments on animals, and conducted in accordance with the guidelines for experiments on animals of the American Physiological Society.

The experiments were performed in anesthetized mongrel dogs of either: sex (weight: $27 \pm 3 \mathrm{~kg}$ (range 22-31)) and chronic total AV block (duration: $5 \pm 2$ weeks [range 2-8]). A detailed description of the experimental model has been previously published. ${ }^{8}$

During the experiments the dogs lied on their side. Unipolar cathodal pacing was performed from the right ventricular endocardium at twice diastolic threshold, using a conventional passive fixation endocardial pacemaker electrode (Slimtine, Vitatron Medical B.V., Dieren, The Netherlands). The VERP was determined with $5 \mathrm{msec}$ accuracy and defined as the longest coupling interval of a premature stimulus, not resulting in ventricular capture. Six surface ECG lead recordings were continuously monitored and stored on a computer system.

Seven experiments were performed in four dogs. One dog was tested twice using both medications and one dog was tested three times: once at baseline only and twice with medication. In all seven experiments IS versus DS was tested without medication, twice at each PCL, except for one experiment in which these measurements were done four times (control: 16 measurements). In four experiments this procedure was repeated after administration of $d$-sotalol (2-3 mg/kg in 
$5 \mathrm{~min}$ ) (8 measurements) and in two experiments after the administration of levcromakalim $(0,01 \mathrm{mg} / \mathrm{kg}$ in 3-5 $\mathrm{min}$ ) (4 measurements). Testing started within 5 min of the administration of medication and lasted no longer than $100 \mathrm{~min}$.

Protocol

At 800 msec steady-state circumstances, the VERP was determined with IS and immediately thereafter with DS. Subsequently the PCL was changed to 350 msec and the protocol was repeated after steady-state circumstances had been reached. After completion the PCL was brought back to $800 \mathrm{msec}$ and the protocol was repeated once more, after steady-state circumstances had been reached. In dogs which received medication the described procedure was repeated.

\section{Incremental Scanning}

Figure 3.1 shows that after reaching steady-state conditions a premature test stimulus $\mathrm{S2}_{1}$, with an initial coupling interval shorter than the VERP, is introduced after every eighth basic drive beat $S 1_{8}$. Since $S 2_{1}$ does not capture, the $S 1 S 1$ interval is not disturbed and steady-state conditions are maintained. Recurrent and abrupt changes in PCL as a result of a capturing S2 are avoided. The coupling intervals of the subsequent premature beats are incremented by $5 \mathrm{msec}$ until capture occurs (Figure 3.2). As soon as the premature stimulus S2 results in a propagated ventricular response the coupling interval for the next premature stimulus
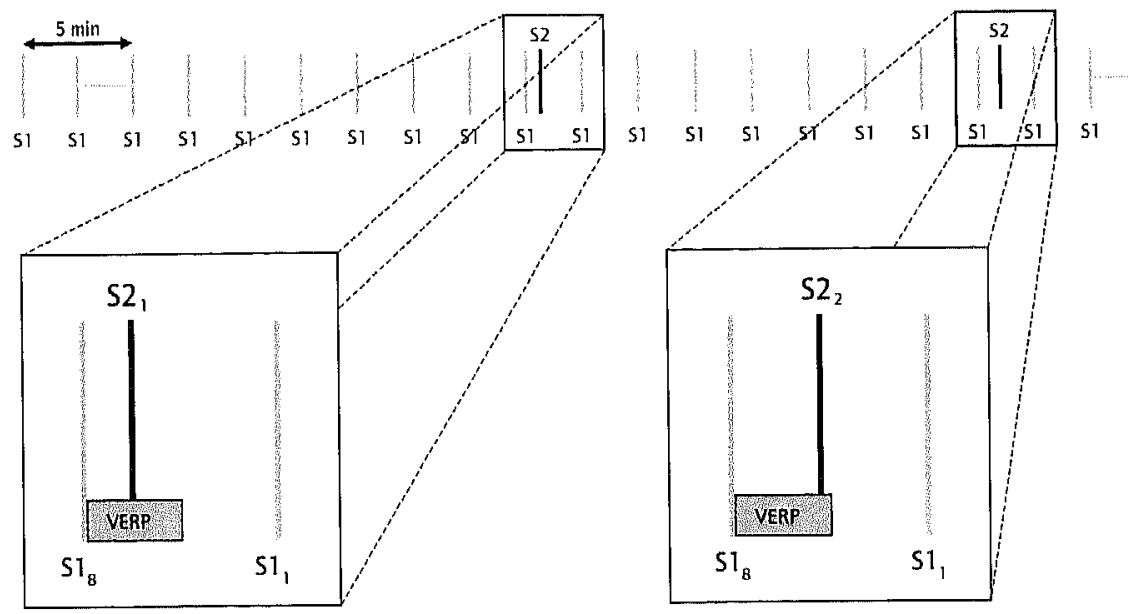

time

Figure 3.1 - Schematic representation of incremental scanning.

After reaching steady-state a premature stimulus 52 is introduced after every eighth 51 . Initially $S 1 S 2<$ VERP, $S 2$ does not capture, the S1S1 drive train is not disturbed, and steady-state conditions are preserved. In subsequent drive trains, SIS2 is incremented by $5 \mathrm{msec}$ until capture occurs. Abbreviation: VERP = ventricular effective refractory period. 


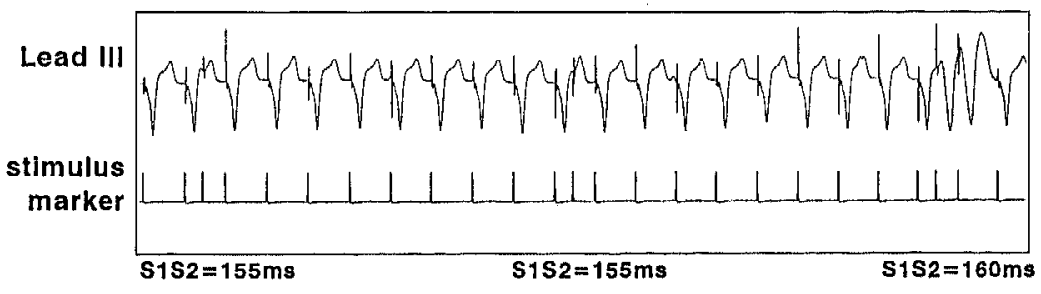

Figure 3.2 - Determination of the VERP at $350 \mathrm{msec}$ steady-state conditions using incremental scanning When $\$ 152$ is incremented to $160 \mathrm{msec}$, capture occurs. During the scanning process the PCL does not change, and steady-state conditions are not disturbed. Abbreviations: VERP = ventricular effective refractory period; $P C L$ = pacing cycle length.

is reduced below the length of the VERP, resulting once again in noncapture of S2 and avoiding abrupt changes in the PCL of the drive train.

\section{Decremental Scanning}

Figure 3.3 shows that after steacly-state conditions are reached, a premature stimulus $\mathrm{S} 2_{1}$, with an initial coupling interval longer than the VERP, is introduced after every eighth basic drive beat $S 1_{8}$. Since $S 2_{1}$ captures, the $S 1 S 1$ interval will
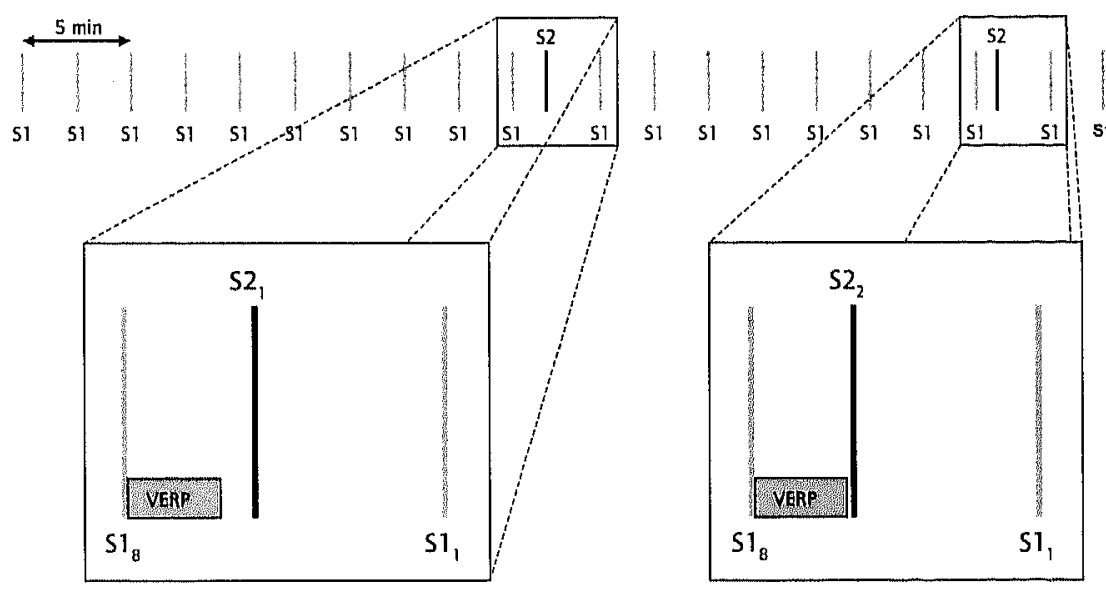

Figure 3.3 - Schematic representation of decremental scanning.

time

After reaching steady-state a premature stimulus $\$ 2$ is introduced after every eighth 51 . Initially $\$ 1$ S2 $>$ VERP S2 caplures, the S1 \$1 drive train is disturbed, and steady-state conditions are not preserved. In subsequent drive trains, S1S2 is decremented by $5 \mathrm{msec}$ until capture is lost. Abbreviation: VERP = ventricular effective refractory period. 
be disturbed. Steady-state conditions will be disturbed and recurrent and abrupt changes in PCL as a result of a capturing S2 will occur. The coupling interval of subsequent premature stimuli is further decremented by $5 \mathrm{msec}$ until capture is lost (Figure 3.4).

Decremental Scanning

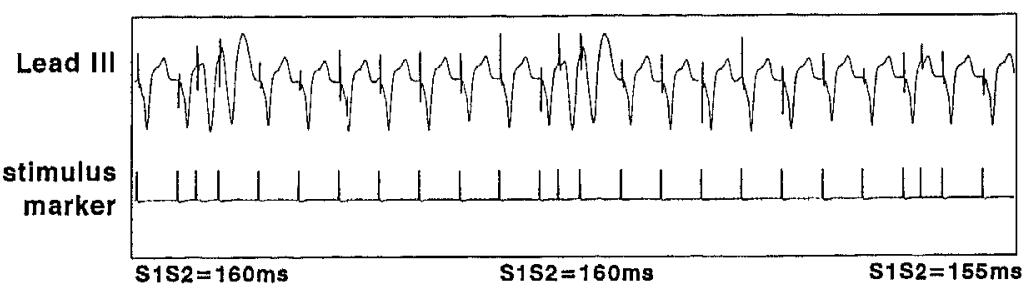

\section{$\mathrm{S} 1 \mathrm{~S} 1=350 \mathrm{~ms}$}

Figure 3.4 - Determination of the VERP at $350 \mathrm{msec}$ steady-state conditions using decremental scanning (the same dog as in Figure 3.2).

When S1S2 is decremented to $155 \mathrm{msec}$, capture is lost. During the scanning process, multiple and abrupt changes in the PCL disturb steady-state conditions. Abbreviations: VERP = ventricular effective refractory period; $\mathrm{PCl}=$ pacing cycle length.

Results

Table 3.1 shows identical steady-state values of the VERP for IS and DS at 800 and $350 \mathrm{msec}$ PCL at baseline (Figures 3.2 and 3.4). Shortening of the VERP due to the change in PCL $\left(\mathrm{VERP}_{800}-\mathrm{VERP}_{350}\right.$ ) was $58 \pm 9 \mathrm{msec}$.

Table 3.2 shows the comparison of IS and DS made before and after d-sotalol. The VERP is prolonged in a reverse use dependent manner, i.e., lengthening of refractoriness is more pronounced at longer cycle lengths compared to shorter ones, ${ }^{9}$ This results in a significant increase in the range of shortening: VERP $800 \mathrm{comt}$ -

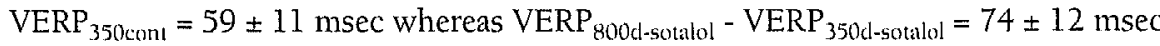
$(\mathrm{P}<0.05$, unpaired Student's $t$-test). More importantly, at the PCLs tested, steadystate values for the VERP using IS or DS show no difference.

Table 3.1 Control values of VERP $(n=16)$.

\begin{tabular}{|c|c|c|c|c|c|c|}
\hline & \multicolumn{3}{|c|}{$\mathrm{PCl}=800 \mathrm{msec}$} & \multicolumn{3}{|c|}{$\mathrm{PCL}=350 \mathrm{msec}$} \\
\hline & IS & DS & IS - DS & is & DS & IS - DS \\
\hline VERP & $226 \pm 15$ & $225 \pm 13$ & $1 \pm 2$ & $768 \pm 10$ & $168 \pm 10$ & $0 \pm 1$ \\
\hline
\end{tabular}

Abbreviations: VERP $=$ ventricular effective refractory period; $P C L=$ pacing cycle length; $\mid S=$ incremental scanning; $D S=$ decremental scanning; $\mid S-D S=$ difference between incremental and decremental scanning. All values in msec as mean $\pm S D$. 
Table 3.2 Values of VERP $(n=8)$, matched control versus $d$-sotalol and their difference.

\begin{tabular}{|c|c|c|c|c|c|c|}
\hline & \multicolumn{3}{|c|}{$\mathrm{PCL}=800 \mathrm{msec}$} & \multicolumn{3}{|c|}{$\mathrm{PCL}=350 \mathrm{msec}$} \\
\hline & is & DS & IS - DS & IS & DS & IS - DS \\
\hline $\mathrm{VERP}_{\text {contr }}$ & $233 \pm 17$ & $231 \pm 15$ & $1 \pm 2$ & $173 \pm 12$ & $173 \pm 12$ & $0 \pm 0$ \\
\hline$V E R P_{\text {d:sotalat }}$ & $261 \pm 21$ & $261 \pm 20$ & $0 \pm 3$ & $187 \pm 13$ & $187 \pm 13$ & $0 \pm 0$ \\
\hline$\triangle V E R P$ & $28 \pm 13$ & $29 \pm 12$ & & $14 \pm 5$ & $14 \pm 5$ & \\
\hline
\end{tabular}

Abbreviations: VERP $=$ ventricular effective refractory period; $\mathrm{PCL}=$ pacing cycle length; $\mid S=$ incremental scanning; $D S=$ decremental scanning; $15-D S=$ difference between incremental and decremental scanning; $\triangle V E R P=V E R P_{\text {disotalo }}-V E R P_{\text {contr }}$. All values in msec as mean $\pm S D$.

Administration of levcromakalim speeds up repolarization via opening of $\mathrm{Ik}_{\mathrm{ATP}}$ channel resulting in shortening of the VERP (Table 3.3). This effect is clearly demonstrated and uniformly present: $V E R P_{800 \mathrm{com}}-\mathrm{VERP}_{350 \mathrm{com}}=51 \pm 8 \mathrm{msec}$

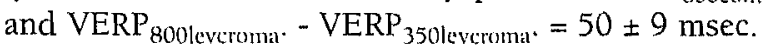

Table 3.3 Values of VERP $(n=4)$, matched control versus levcromakalim and their difference.

\begin{tabular}{|c|c|c|c|c|c|c|}
\hline & \multicolumn{3}{|c|}{$\mathrm{PCL}=800 \mathrm{msec}$} & \multicolumn{3}{|c|}{$\mathrm{PCl}=350 \mathrm{msec}$} \\
\hline & IS & DS & IS - DS & is & DS & IS - DS \\
\hline VERP contr & $215 \pm 9$ & $215 \pm 9$ & $1 \pm 2$ & $164 \pm 3$ & $163 \pm 3$ & $0 \pm 0$ \\
\hline VERP Iavcionakallin & $206 \pm 8$ & $206 \pm 8$ & $0 \pm 3$ & $156 \pm 5$ & $156 \pm 5$ & $0 \pm 0$ \\
\hline$\triangle V E R P$ & $-9 \pm 5$ & $-9 \pm 5$ & & $-8 \pm 7$ & $-6 \pm 8$ & \\
\hline
\end{tabular}

Abbreviations: $\mathrm{VERP}=$ ventricular effective refractory period; $\mathrm{PCl}=$ pacing cycle length, iS $=$ incremental scanning; $D S=$ decremental scanning; $I S-D S=$ difference between incremental and decremental scanning $\triangle V E R P=V E R P_{\text {laveromakalim }}-V E R P_{\text {contr. }}$ All Values in msec as mean $\pm 5 D$.

\section{Discussion}

\section{Intertrain Pause}

In our protocol no intertrain pause was used. By using an intertrain pause the shortening of the VERP caused by the eight beat drive train and the premature stimulus partially recovers. Different intertrain pauses lead to different stages of recovery and, therefore, to different starting values for the VERP of subsequent drive trains. ${ }^{10}$ Furthermore, the basic drive train of eight beats never reaches new steady-state conditions since the maximum cumulative effect is not reached. $1,11,12$ 
Longer values for VERP have been reported using an intertrain pause comparing them with VERP values with no or shorter intertrain pauses. ${ }^{10}$

\section{Noncapturing Premature Beat}

In IS a noncapturing beat is used to scan the VERP. The influence of this noncapturing beat on the VERP was studied by Janse et al. ${ }^{1}$ and Morady et al. ${ }^{4}$ These authors reported that noncapturing beats in drive cycles preceding the premature stimulus do not influence the length of the VERP.

\section{IS Versus DS}

Comparing IS versus DS, Morady et al. ${ }^{+}$found no difference in values for the VERP, whereas Beauregard et al. ${ }^{5}$ found longer values using IS compared to

[ 44 ] DS. This apparent discrepancy could be contributed to the absence of an intertrain pause in the first study whereas in the second study an intertrain pause (3-4 sec) was used.

Theoretically, however, a longer VERP for IS compared to DS should be expected in the absence of an intertrain pause. Since, in the decremental protocol, the coupling interval of the capturing premature beat, is shorter than the coupling interval of the basic train, there will be an extra shortening of the VERP. The reason for not finding significant differences between IS and DS in our experiments could be due to: (1) the compensating effect of the eight beat basic drive train; and/or (2) the limited resolution of 5 msec used to determine the VERP.

\section{Pharmacologic Conditions}

The effect of medication was evident in all cases (Tables 3.2 and 3.3). Since we did not observe a change in drug effect during the course of the experiment, we assume that drug levels were constant. As anticipated, VERP lengthened in a reverse use dependent manner after the administration of d-sotalol, ${ }^{9}$ whereas levcromakalim shortened the VERP uniformly. ${ }^{7}$ No differences for the VERP were found comparing IS to DS after the administration of medication that affects the VERP.

\section{Clinical Implications}

IS and DS without intertrain pauses allow reproducible measurements of the VERP under steady-state conditions at different PCLs with or without medication that affects the VERP. However, when the arrhythmogenicity of the scanning protocol should be kept at a minimum, IS has the additional advantage of avoiding frequent and abrupt changes in PCL. 


\section{Conclusion}

No differences in VERP were found comparing IS to DS at two PCLs with or without medications that affect the VERP.

\section{References}

1. Janse MJ, van der Steen $A B$, van Dam RT, et al. Refractory period of the dog's ventricular myocardium following sudden changes in frequency. Circ Res 1969; 24:251-262.

2. Ross DL, Farre J, Bär FW, et al. Comprehensive clinical electrophysiologic studies in the investigation of documented or suspected tachycardias; time, staff, problems and costs. Circulation 1980; 61:1010-1016.

3. Brugada P, Wellens HJ. Standard diagnostic programmed electrical stimulation protocols in patients with paroxysmal recurrent tachycardias. PACE 1984; 7:1121-1128.

4. Morady F, Kadish AH, Kushner JA, et al. Comparison of ventricular refractory periods determined by incremental and decremental scanning of an extrastimulus. PACE 1989; 12:546-554.

5. Beauregard LM, Friehling TD, Marinchak RA, et al, Measurement of human ventricular effective refractory periods: effect of ineremental versus decremental extrastimulation. J Cardiovasc Electrophysiol 1990; 1:506-511.

6. Taggert P, Sutton P, Donaldson R. cl-Sotalol: a new potent class III anti-arrhythmic agent. Clin Sci 1985; 69:631-636.

7. Spinelli W, Follmer $C$, Parsons R, et al. Ellects of cromakalim, pinacidil and nicorandil on cardiac refractoriness and arterial pressure in open-chest dogs. Eur J Pharmacol 1990; 179:243252.

8. Vos MA, Deursen van RT, Gorgels AP, el al. R56865, an antiarrhythmic drug with class $l 11$ effects that terminates ouabin induced ventricular tachycardia in an inverse rate-dependent manner. Cardiovasc Res 1993; 27:1491-1497.

9. Rulfy R. Sotalol. J Cardiovasc Electrophysiol 1993; 4:81-98.

10. Morady $\mathrm{F}$, Kadish $\mathrm{AH}$, Rosenheck $\mathrm{S}$, el al. Effect of the intertrain pause on the ventricular effective refiactory period measured by the extrastimulus technique. PACE 1990; 13:405-409.

11. Morady F, Kadish A., Toivonen LK, et al. The maximum effect of an increase in rate on human ventricular refractoriness. PACE 1988; 11:2223-2234.

12. Brownstein SL, Blackwell WH, Welch WJ, et al. Cumulative effects of cycle length on ventricular refricloriness in man. Am Heart J 1990; 119:324-330. 


\section{Chapter 4}

\section{Inter- and Intraindividual Variations in Shortening of Ventricular Effective Refractory Period After an Abrupt Decrease in Pacing Cycle Length}

Hendrik M. Leerssen, Marc A. Vos, Karel den Dulk, Jolanda van der Zande, Malcolm J. Begemann, * and Hein J.J. Wellens.

Department of Cardiology, Cardiovascular Research Institute Maastricht, Maastricht University, The Netherlands, and *Vitatron Medical B.V., Dieren, The Netherlands.

Published in PACE 1994; 17(Pt. II):2079-2083 


\section{Abstract}

Introduction: After an abrupt decrease in pacing cycle length (PCL) the ventricular effective refractory period (VERP) shortens. The pacing protocol needed to determine accurate and reproducible values for the VERP during this process is elaborate and time consuming.

Methods and Results: In this study steady-state values of VERP at 800 and $350 \mathrm{msec}$ PCL and dynamic values of VERP due to an abrupt change in PCL from 800 to $350 \mathrm{msec}$ were determined. This was done for eleven different dogs to test the interindividual variation and repetitively in the same dog to test the intraindividual variation. The results for steady-state and dynamic values of the VERP show a wide range in both groups.

[48] Conclusion: Accurate prediction of steady-state and dynamic values of VERP based on previous measurements is not possible. 


\section{Introduction}

After an abrupt increase in heart rate the ventricular effective refractory period (VERP) shortens to reach new steady-state conditions. This shortening is initially pronounced over a few beats and gradually decreases thereafter ${ }^{l-3}$ To obtain beat-to-beat information on dynamics of the VERP after a sudden increase in heart rate an elaborate and time consuming protocol is required. ${ }^{2}$ Therefore only limited information is available with respect to the exact dynamics of shortening and reproducibility. ${ }^{3}$

In this study steady-state values of VERP at 800 and $350 \mathrm{msec}$ pacing cycle length (PCL) were determined. Furthermore, the dynamics of the VERP as a result of an abrupt change in PCL from 800 to 350 msec were studied on a beat-to-beat basis. This was done in different dogs to study the interindividual variation and repetitively in the same dog to study the intraindividual variation.

\section{Methods}

This protocol was approved by the local ethical committee for experiments on animals, and conducted in accordance with the guidelines for experiments on animals of the American Physiological Society.

The experiments were performed in 11 anesthetized mongrel dogs of either sex (weight: $26 \pm 4 \mathrm{~kg}$ (range 21-33)) with chronic (duration: $5 \pm 2$ weeks [range 2 - 8)) atrioventricular-block. During the experiments the dogs lied on their side. A detailed description of the model has been published previously. ${ }^{4}$ In each dog a single experiment was performed to test the interindividual variation (Table 4.1). To test the intraindividual variation, three dogs $(3,4$, and 8$)$ were tested twice and one $\operatorname{dog}(9)$ was tested three times (Table 4,2). The time interval between consecutive experiments in the same dog was at least 1 week.

Unipolar cathodal pacing was performed from the right ventricular endocardium at twice diastolic threshold with a conventional passive fixation endocardial pacemaker electrode (Slintine, Vitatron Medical B.V., Dieren, The Netherlands). ECG lead recordings were continuously monitored and stored on a computer.

\section{Steady-State Values of VERP}

The VERP was determined using steps of $5 \mathrm{msec}$ and was defined as the longest coupling interval of a premature stimulus not resulting in ventricular capture.

Steady-state values of the VERP were determined at $800\left(\mathrm{VERP}_{800}\right)$ and 350 msec PCL $\left(V_{E R P}{ }_{350}\right)$. The total amount of shortening due to the abrupt change in

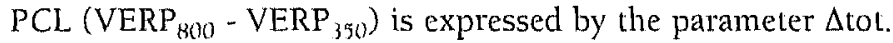

To determine VERP ${ }_{800}$ and VERP ${ }_{350}$, incremental scanning with no inter- 
Table 4.1 Steady state and dynamic values of VERP for the "inter - group",

\begin{tabular}{|c|c|c|c|c|c|c|c|c|}
\hline \multirow[b]{2}{*}{$\operatorname{dog}$} & \multirow[b]{2}{*}{$\begin{array}{l}\mathrm{VERP}_{800} \\
\text { (msec) }\end{array}$} & \multirow[b]{2}{*}{$\begin{array}{l}\text { VERP }_{350} \\
\text { (msec) }\end{array}$} & \multirow[b]{2}{*}{$\begin{array}{c}\Delta \text { tot } \\
\text { (msec) }\end{array}$} & \multicolumn{2}{|l|}{$\Delta 3$} & \multicolumn{2}{|l|}{$\Delta 20$} & \multirow[b]{2}{*}{$\underset{\text { (msec) }}{\mathrm{RR}}$} \\
\hline & & & & (msec) & $\%$ & (msec) & $\%$ & \\
\hline 1 & 255 & 185 & 70 & 25 & 36 & 40 & 57 & 1980 \\
\hline 2 & 245 & 180 & 65 & 25 & 38 & 35 & 54 & 1600 \\
\hline 3 & 220 & 175 & 45 & 10 & 22 & 25 & 56 & 2500 \\
\hline 4 & 215 & 150 & 65 & 15 & 23 & 30 & 46 & 1200 \\
\hline 5 & 235 & 175 & 60 & 20 & 33 & 35 & 58 & 860 \\
\hline 6 & 210 & 160 & 50 & 5 & 70 & 20 & 40 & 1200 \\
\hline 7 & 225 & 165 & 60 & 20 & 33 & 35 & 58 & 1400 \\
\hline 8 & 235 & 185 & 50 & 10 & 20 & 15 & 30 & 1800 \\
\hline 9 & 230 & 170 & 60 & 20 & 33 & 30 & 50 & 1225 \\
\hline 10 & 215 & 160 & 55 & 15 & 27 & 30 & 55 & 1000 \\
\hline 11 & 240 & 180 & 60 & 20 & 33 & 30 & 50 & 1500 \\
\hline mean $\pm S D$ & $230 \pm 74$ & $171 \pm 11$ & $58 \pm 8$ & $17 \pm 6$ & $28 \pm 9$ & $30 \pm 7$ & $50 \pm 9$ & $1480 \pm 470$ \\
\hline range & $210-255$ & $150-185$ & $45-70$ & $5-25$ & $10-38$ & $15-40$ & $30-58$ & $860-2500$ \\
\hline
\end{tabular}

Abbreviations: $\mathrm{VERP}=$ ventricular effective refractory periodi $\mathrm{VERP} \mathrm{Bn0}_{800}$ and $\mathrm{VER} \mathrm{P}_{350}=\mathrm{VERP}$ at 800 and 350 msec pacing cycle length; $\Delta$ tot $=V V^{2} P_{800}-V E R P_{3,0 ;} \Delta 3$ and $\Delta 20=$ the change in VERP after 3 and 20 beats with $350 \mathrm{msec}$ pacing cycle length expressed in $\mathrm{msec}$ and as $\%$ of $\Delta$ tot; $R R=$ interval of idioventricular rate.

train pause was used (Figure 4.1). A premature test stimulus S2, with an initial coupling interval shorter than VERP was introduced after every eighth basic drive beat S1. Since S2 did not capture, steady-state conditions could be reproducibly maintained, and abrupt changes in rate as a result of a capturing S2 were avoided. The coupling interval of the subsequent $\$ 2$ was incremented by $5 \mathrm{msec}$ until capture occurred.

\section{Dynamics of VERP}

The shortening of VERP due to the abrupt decrease in PCL was studied on a beat-to-beat basis using the procedure outlined in Figure 4.2. To scan the length of the VERP, a premature test pulse was introduced after the last beat of the 350 msec drive train. The initial coupling interval of the test pulse was chosen to be shorter than the VERP, therefore not resulting in ventricular capture (Figure 4.2, upper panel). The PCL is then brought back to $800 \mathrm{msec}$. To confirm that steadystate circumstances had been restored, on-line monitoring of the stimulus- $T$ interval $^{5}$ was used. Using a custom-build stimulator (Vitatron Medical B.V., Dieren, 
Table 4.2 Steady state and dynamic values of VERP for the "intra - group".

\begin{tabular}{|c|c|c|c|c|c|c|c|c|c|}
\hline $\operatorname{dog}$ & VERP $_{B 00}$ & Range $_{800}$ & VERP $_{350}$ & Range $_{350}$ & $\Delta 3$ & Range $_{\Delta 3}$ & $\Delta 20$ & Range $_{\Delta 20}$ & RR \\
\hline \multirow[t]{2}{*}{3} & 220 & & 175 & & 10 & & 25 & & 2500 \\
\hline & 245 & 25 & 190 & 15 & 15 & 5 & 30 & 5 & 2300 \\
\hline \multirow[t]{2}{*}{4} & 215 & & 150 & & 15 & & 30 & & 1200 \\
\hline & 200 & 15 & 150 & 0 & 15 & 0 & 25 & 5 & 1500 \\
\hline \multirow[t]{2}{*}{8} & 235 & & 185 & & 10 & & 15 & & 1800 \\
\hline & 210 & 25 & 165 & 20 & 10 & 0 & 20 & 5 & 1600 \\
\hline \multirow[t]{3}{*}{9} & 230 & & .170 & & 20 & & 30 & & 1225 \\
\hline & 260 & & 180 & & 35 & & 45 & & 1200 \\
\hline & 225 & 35 & 165 & 15 & 15 & 20 & 30 & 15 & 1185 \\
\hline mean & & 25 & & 13 & & 6 & & 8 & \\
\hline range & & $15-35$ & & 0.20 & & 0.20 & & $5 \cdot 15$ & \\
\hline
\end{tabular}

Abbreviations: $\mathrm{VERP} \approx$ ventricular effective refractory period; VERP 800 and VERP $350=\mathrm{VERP}$ at 800 and 350 msec pacing cycle length; Range $e_{800}$ and Range $e_{350}=$ the range in values of $V E R P_{800}$ and $V E R P_{350}$ for each incividual dog; $\Delta 3$ and $\Delta 20=$ the change in VERP after 3 and 20 beats with 350 msec pacing cycle length; Range $_{\Delta 3}$ and Range ${ }_{\Delta 20}=$ the range in values of $\Delta 3$ and $\Delta 20$ in each individual dog; $R R=$ interval of idioventricular rate. Dog numbers correspond with dog numbers in Table 4.1. All values in msec.

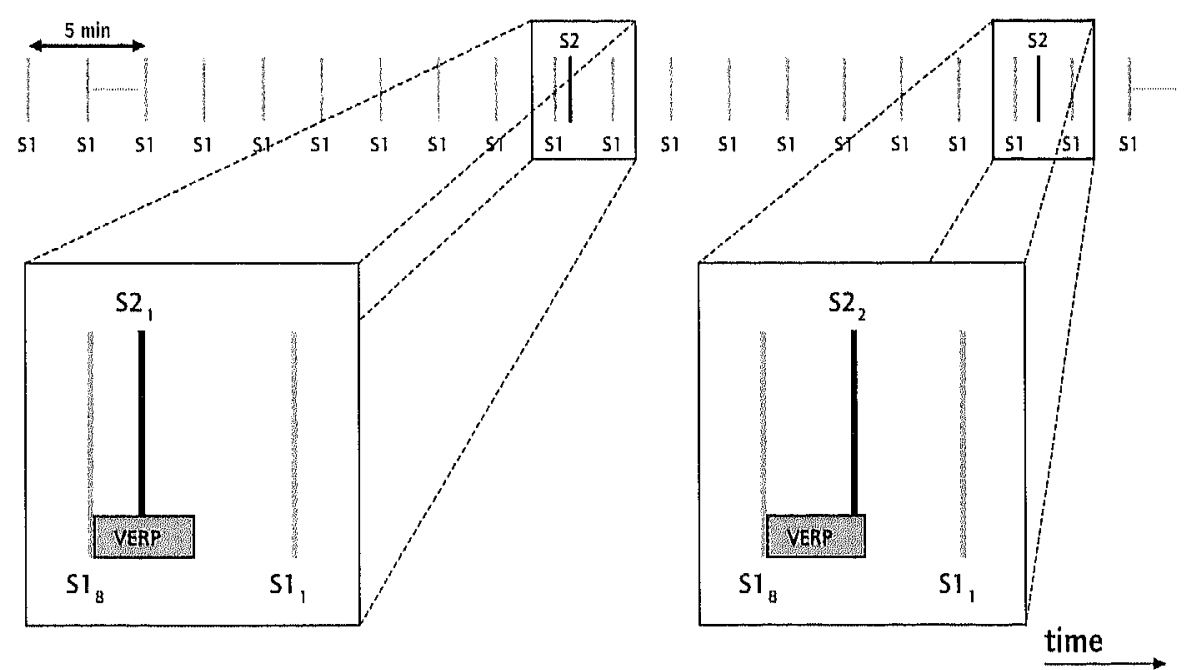

Figure 4.1 - Schematic representation of incremental scanning.

After reaching steady-state, a premature stimulus $\$ 2$ is introduced after every eighth $\$ 1$. Initially $\$ 1 S 2<$ VERP, S2 does not capture, the S1S1 drive train is not disturbed, and steady-state conditions are preserved. In subsequent drive trains 5152 is incremented by $5 \mathrm{msec}$ until capture occurs. Abbreviation: VERP = ventricular effective refractory period. 


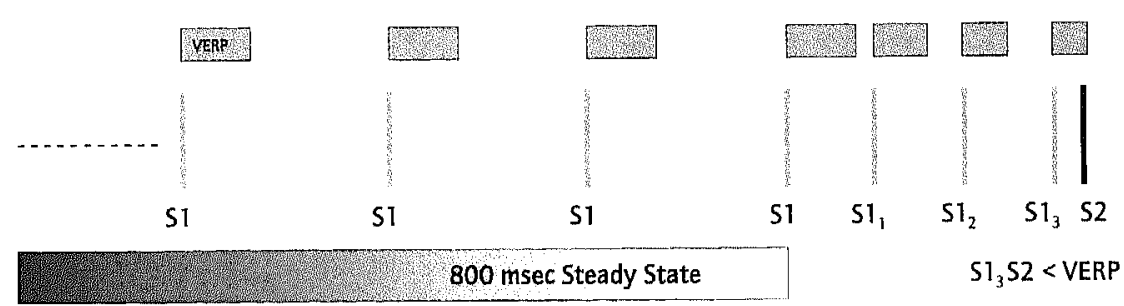

time

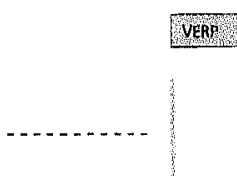

S1

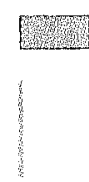

ST
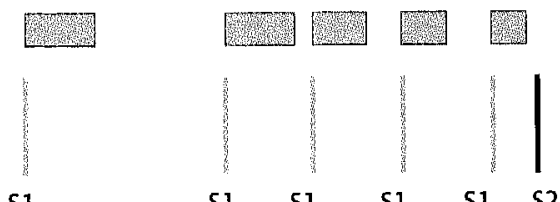

S1

$51 \quad 51$

$\begin{array}{llll}\mathrm{SI}_{2} & \mathrm{SI}_{3} & \mathrm{~S} 2\end{array}$

$\mathrm{SI}_{3} \mathrm{SZ}>\mathrm{VERP}$

Figure 4.2 - The protocol to study beat-to-beat changes in VERP after an abrupt decrease in PCL from 800 to $350 \mathrm{msec}$, e.g., the change in VERP after three beats.

Starting at 800 msec steady-state conditions a 3 beat $350 \mathrm{msec}$ drive train $\left(S 1_{1}, \mathrm{Sl}_{2}\right.$, and $\left.S 1_{3}\right)$ is delivered. This drive train is followed by a test stimulus (S2) to scan the VERP. Initially SIS2 < VERP (upper panel). After restoring $800 \mathrm{msec}$ steady-state conditions, the same drive train is delivered except for the fact that the S1S2 is incremented by $5 \mathrm{msec}$. This pacing sequence is repeated until $51 \mathrm{S2}>$ VERP (lower panel). This procedure can be repeated for $350 \mathrm{msec}$ drive trains of any length, enabling a beat-to-beat scan of the dynamics of the VERP after the abrupt decrease in $\mathrm{PCL}$. Abbreviations: $\mathrm{VERP}=$ ventricular effective refractory period; $P C L=$ pacing cycle length.

The Netherlands) the actual value for the stimulus-T interval could be monitored constantly and compared to the value found for steady-state circumstances. Subsequently the coupling interval between the last beat in the $350 \mathrm{msec}$ drive train and the premature test pulse was incremented by $5 \mathrm{msec}$. The procedure was repeated until capture occurred, i.e., the coupling interval of the premature test stimulus was longer than VERP (Figure 4.2, lower panel). To compare the dynamics of the VERP for the inter- and intraindividual groups, we arbitrarily selected two points of the dynamic curve of VERP representing the change in the VERP at $3(\Delta 3)$ and 20 beats $(\Delta 20)$, after the abrupt change in PCL from 800 to $350 \mathrm{msec}$.

\section{Results}

For all experiments $(n=16), V E P_{800}$ was $229 \pm 17 \mathrm{msec}$ and VERP 350 was $171 \pm 12 \mathrm{msec}$, yielding a $\Delta$ tot of $58 \pm 9 \mathrm{msec}$. 
Interindividual Group

The values of VERP 800 and VERP ${ }_{350}$ are shown in Table 4.1. Both parameters have a wide variation. Furthermore, the length of $V_{E R P}{ }_{800}$ is not related to the cycle length of the idioventricular rhythm $(R R)(r=0.3$, linear regression and correlation). Although the change in PCL for all experiments is identical, $\Delta$ tot shows a wide variation ranging from $45-70 \mathrm{msec}$. Furthermore, $\Delta$ tot is not related to $R R$ $(\mathrm{r}=0.4)$. The dynamic behavior shows a similar pattern: a large variation in $\Delta 3$ ( $29 \pm 8 \%$ of $\Delta t o t)$ and $\Delta 20(51 \pm 8 \%$ of $\Delta$ tot $)$. In addition, identical values for $\Delta$ tot did not always correspond to identical values for $\Delta 3$ and/or $\Delta 20$, once again indicating different dynamics of VERP among different dogs.

\section{Intraindividual Group}

In addition to VERP ${ }_{800}, V V P_{350}$, Table 4.2 shows the parameters range $e_{800}$ and range 350 , which represent the largest difference found between the different values for an individual dog. Again VERP ${ }_{800}$ and VERP 350 display wide variations, yielding a range s00 $_{0}$ of $35 \mathrm{msec}$ in dog 9 , with a mean difference of $25 \mathrm{msec}$ for all dogs. Since the cycle length of the idioventricular rate was stable for consecutive experiments in one dog this was considered not to be a confounding factor.

\section{Discussion}

To determine steady-state and clynamic values of the VERP, an elaborate and time consuming protocol is needed. ${ }^{2}$ This may explain why only limited data are available, especially with respect to the dynamics of the VERP. Moreover, in the few reports that are available, the authors use different protocols in which no steady-state circumstances were established before the drive trains to determine the VERP were delivered. $1,3,0-8$ For example, Han and Moe ${ }^{1}$ reported $50 \%$ of the total shortening after two short cycles and completion of shortening after 14 cycles, while Janse et al. ${ }^{2}$ reported $27 \%$ of the total shortening after 1 beat and 40 $45 \%$ of the total shortening after two beats. New steady-state conditions were reported to be reached after a few (hundred) beats, ranging between $4-388.2,7$ Therefore, data are difficult to compare and, more importantly, seem not to be reproducible.

Whether this very pronounced variability is primarily related to the methods used or reflects the variability of the individuals tested, was the subject of this investigation. In our protocol we tried to minimize methodological variation by ascertaining that steady-state conditions at the basic PCL $800 \mathrm{msec}$ were continuously reached. For this we waited until the actual stimulus-T interval was equal to the value found at $800 \mathrm{msec}$ steady-state circumstances. Furthermore, incremental scanning was used to reduce interference of capturing premature stimuli to a minimum.

Nevertheless, we demonstrated a large interindividual variation and more 
importantly a marked intraindividual variation over weeks for steady-state and dynamic parameters. The variation was not related to RR and seemed to be independent of $\Delta$ tot. RR Was relatively constant for subsequent experiments in the same dog, suggesting comparable levels of autonomic tone during anesthesia.

Oscillations in the VERP after an abrupt change in PCL have been found and could explain the wide variation. ${ }^{2,9}$ However, this does not seem likely, since oscillations only occur after an abrupt change to a shorter cycle length that approaches the VERP of the baseline cycle length. In our experiments the VERP ${ }_{800}$ was $229 \pm 7 \mathrm{msec}$ which is much shorter than the short PCL ( $350 \mathrm{msec}$ ).

Our dogs had biventricular hypertrophy due to volume overload created by the chronic atrioventricular block. Hypertrophy is known to lengthen repolarization parameters. Denardo et al. ${ }^{10}$ compared the dynamics of the VERP in patients [54] with structurally normal hearts with that of patients with hypertrophy with or without chronic heart failure. Although the VERP was lengthened, the ability of the VERP to adapt to a sudden increase in heart rate was not impaired in the latter groups. Therefore, hypertrophy does not explain our findings of marked variation of the VERP. The fact that in later experiments equal or even shorter steadystate values for VERP were found (Table 4.2) indicates that the degree of hypertrophy was stable.

When accurate information on the VERP in patients is needed and a study using the appropriate methods is not feasible, other parameters that show good correlation with the VERP can be of use. It has been shown that monophasic action-potential duration ${ }^{11}$ and the stimulus-T interval ${ }^{12,13}$ can be used to predict the VERP. Both parameters have the advantage of being measurable without the use of premature stimuli. The stimulus- $T$ method has the additional advantage of being measurable with chronically implanted leads.

\section{Conclusion}

The steady-state and dynamic values of the VERP determined at one point in time have little predictive value for the determination of the VERP under comparable conditions Iater in time. Therefore, when accurate information on the VERP is needed, other well correlated and on-line available parameters such as the stimulus-T interval, should be considered.

\section{References}

1. Han J, Moe GK. Cumulative effects of cycle length on refractory periods in cardiac tissues. Am J Physiol 1969; 217:106-109.

2. Janse MJ, van der Steen $A B$, van Dam RT, et al. Refractory period of the dog's ventricular myocardium following sudden changes in frequency. Circ Res 1969; 24:251-262. 
3. Brownstein SL, Blackwell WH, Welch WJ, et al. Cumulative effects of cycle length on ventricular refractoriness in man. Am Heart J 1990; 119:324-330.

4. Vos MA, Deursen van RT, Gorgels AP, et al. R56865, an antiarrhythmic drug with class III effects that terminates ouabin induced ventricular tachycardia in an inverse rate-dependent manner. Cardiovasc Res 1993; 27:1491-1497.

5. Rickards AF, Norman J. Relation between QT interval and heart rate. New design of physiologically adaptive cardiac pacemaker. Br Heart J 1981; 45:56-61.

6. Wiener I, Kunkes S, Rubin D, et al, Effects of sudden change in cycle length on human atrial, atrioventricular nodal and ventricular refractory periods. Circulation 1981; 64:245-248.

7. Morady $F$, Kadish $A H$, Toivonen $L K$, et al. The maximum effect of an increase in rate on human ventricular refractoriness. PACE 1988; 11:2223-2234.

8. Rosenheck S, Schmaltz S, Kadish AH, et al. The eflect of quinidine and nexiletine on the adaptation of ventricular refractoriness 10 an increase in heart rate. Am Heart J 1991; 121:512-517.

9. Marchlinski FE. Characterization of oscillations in ventricular refractoriness in man after an abrupt increment in heart rate. Circulation 1987; 75:550-556.

10. Denardo SJ, Stevenson WG. Is the adaptation of right ventricular refractoriness to an abrupt increase in heart rate impaired in chronic heart failure? Am Heart J 1989; 117:585-589.

11. Morgan JM, Cunningham AD, Rowland E. Relationship of the effective refractory period and the monophasic action-potential cluration after a step increase in pacing frequency. PACE 1990 13:1002-1008.

12. den Dulk K, Leerssen HM, Vos MA, et al. Applicability of the stinulus-T interval for antitachycardia pacing. PACF 1991; 14(Pt. II):1757-1761.

13. Leerssen HM, den Dulk K, Vos MA, et al. Individual variation in dynamic behavior of ventricular effective refractory period and action-potential duration following rate changes as assessed by the stimulus-T technique. (abstract) Eur Heart J 1993; 14(suppl):87. 


\section{Chapter 5}

\section{Steady-State and Dynamic Behavior of Ventricular Repolarization and Refractoriness in the Dog:}

The Effect of Multiple Cycle-Length Changes and d-Sotalol Administration

Hendrik M. Leerssen, Marc A. Vos, Karel den Dulk, Jolanda van der Zande,

Arno M.M. Muijtjens, ${ }^{*}$ Malcolm J. Begemann, ${ }^{*}$ and Hein J.J. Wellens.

Department of Cardiology, * Department of Medical Informatics, Cardiovascular Research Institute Maastricht, Maastricht University, The Netherlands, and * *Vitatron Medical B.V., Dieren, The Netherlands.

Published in PACE 1998; 21:1766-1777. 


\section{Abstract}

Introduction: In anesthetized dogs with chronic, complete atrioventricular (AV) block we studied the characteristics of ventricular repolarization and refractoriness.

Methods and Results: We determined: (1) steady-state values of ventricular effective refractory period (VERP), action-potential duration (APD), and stimulus-T interval (STI) before and after d-sotalol treatment at various pacing cycle lengths (PCLs); and (2) the dynamics of VERP, APD, and STI before and after d-sotalol treatment after the abrupt PCL decreases. VERP, APD, and STI showed a normal frequency dependency. All three parameters increased significantly after d-sotalol administration. During steady-state and dynamic measurements, STI was always longer than APD and APD was always longer than VERP in an individual animal, irrespective of PCL and conditions. Standard deviations of steady-state and dynamic values indicated a considerable interindividual variation. However, the dynamics of VERP, APD, and STI after an abrupt decrease in PCL were highly correlated (linear regression analysis: $\mathrm{r}^{2} \geq 0.93$ ). The best mathematical model to describe these dynamics was a bi-exponential model $\left(r^{2} \geq 0.98\right)$ with a very short first and a much longer second time constant.

Conclusion: We found that there is a very consistent relation between VERP, APD, and STI, not only during steady-state but also in the dynamic situation after various abrupt PCL decreases. This relation does not change after the administration of d-sotalol. STI could be used to predict steady-state and dynamic values of VERP and APD. Since STI can be made available on-line in implantable pacing systems this could lead to the development of new features in these devices. 
Introduction

The onset of ventricular tachycardia (VT) is usually accompanied by an abrupt increase in heart rate. Repolarization dynamics, however, are considered to be gradual, taking several minutes for the heart to adjust to the faster rhythm. 1,2

The shortening in duration of repolarization at the onset of VT is accompanied by a change in the antitachycardia pacing (ATP) ${ }^{3}$ termination window. This window starts at the end of refractoriness and ends the latest moment ATP can be successful. In the dynamic situation after the onset of VT, on-line monitoring of the ventricular effective refractory period (VERP) or the action-potential duration (APD) in implantable pacing systems could enable these devices to adjust coupling intervals during ATP on a beat-to-beat basis. However, to determine the dynamics of VERP an elaborate and time consuming pacing protocol, with multiple rate changes ${ }^{1}$ is needed and APD can only be measured after the introduction of a special catheter, which is not stable chronically. ${ }^{4}$ For these reasons we tested to what extent the stimulus-T interval (STI) (= interval from moment of stimulation until T-wave sensing of the endocardially-evoked response) which can be made available on-line in implantable pacing systems via a normal chronically stable pacemaker electrode, ${ }^{5}$ could be used to characterize and predict VERP and APD on a beat-to-beat basis, especially under circumstances when the rate is suddenly increased.

Until now, steady-state and dynamic behavior of repolarization and refractoriness has been studied using VERP, 1,2,6-8 APD, $4,9-13$ or QT interval. ${ }^{14}$ For multiple steady-state conditions the correlation between VERP and activation recovery interval (ARI) ( = interval between most rapid voltage decrease in QRS and maximum rate of increase in T-wave in electrogram), ${ }^{15}$ APD and ARI, ${ }^{16}$ and APD and $\mathrm{STI}^{17}$ has been studied. In the dynamic situation after a step increase in pacing frequency only the correlation between VERP and APD ${ }^{18}$ has been tested.

To our knowledge this is the first study in which steady-state values and dynamic behavior of VERP, APD, and STI before and during the onset and continuation of a simulated VT are determined and compared. The experiments were performed in dogs with chronic complete atrioventricular (AV) block in the absence or presence of the class III agent d-sotalol ${ }^{19-22}$ using various abrupt decreases in pacing cycle length (PCL). Furthermore we determined the optimal mathematical model to characterize the dynamics of VERP, APD, and STI after these PCL decreases.

\section{Methods}

This protocol was approved by the local ethical committee for experiments on animals, and conducted in accordance with the guidelines for experiments on animals of the American Physiological Society. 
Definitions

Ventricular effective refractory period (VERP): The longest coupling interval of a premature stimulus at twice the late diastolic threshold, not resulting in a propagated ventricular response.

Action-potential duration (APD): The interval from the beginning of the upstroke of the local activation to the end of repolarization (APD100).

Stimulus-T interval (STI): The interval that starts at the moment of stimulation and ends at the moment of T-wave sensing in the downgoing slope of the T-wave of the endocardially-evoked response. STI measurement was standardized using the following procedure. The amplitude of the evoked T-wave was determined by decreasing the sensitivity setting. Disappearance of the I-wave marker at a certain setting for the T-wave sensitivity indicated the moment of undersens-

[60] ing. The value for T-wave sensitivity was programmed to half the value at which undersensing occurred.

Preparation

In 14 anesthetized mongrel dogs of either sex (weight: $26 \pm 4 \mathrm{~kg}$, (range 21 - 33)), with induced complete AV block (duration AV block: $4 \pm 2$ weeks, [range 2 - 7)), 23 experiments were performed. At least two weeks before the actual experiment AV block was induced by injecting 37\% formaldehyde into the AV junction without atriotomy after a right thoracotomy. ${ }^{23} \mathrm{~A}$ permanent pacemaker electrode (Slimtine, Vitatron Medical B.V., Dieren, The Netherlands) was inserted endocardially into the apical part of the right ventricle.

Both the AV block operation and the actual experiment were performed under sterile anesthetized conditions enabling recovery of the dogs. Furthermore an antibiotic (1000 mg ampicillin) was given pre-operatively and post-operatively. After premedication was given intramuscularly, anesthesia was induced by intravenous sodium pentobarbitone $(20 \mathrm{mg} / \mathrm{kg})$. The dogs were artificially ventilated through a cuffed endotracheal tube with a mixture of oxygen, nitrous oxide, and halothane (vapor concentration $0.5-1 \%$ ). Ventilation was adjusted on the basis of continuous monitoring of the carbon dioxide concentration of the expired air. A thermal mattress was used to maintain body temperature at approximately $37^{\circ}$ Celsius.

Protocol

The experiments were performed to determine:

(1) Steady-state values of VERP, APD, and STI at 1000, 800, 500, and 350 msec PCL. The effect of d-sotalol treatment ( $2-3 \mathrm{mg} / \mathrm{kg}$ in $5 \mathrm{~min})$ was determined at 800 and $350 \mathrm{msec}$ PCL.

(2) The dynamic behavior of VERP, APD, and STI after an abrupt decrease in PCL from 800 to $350 \mathrm{msec}$. This was done before and after d-sotalol treatment (2-3 $\mathrm{mg} / \mathrm{kg}$ in $5 \mathrm{~min}$ ). The dynamic behavior of STI was also determined after an abrupt PCL decrease from $1250-350 \mathrm{msec}$. 
Using these data:

(1) The total amount of shortening $(\Delta)$ of VERP, APD, and STI after the abrupt PCL decrease $800-350 \mathrm{msec}$ and $1000-500 \mathrm{msec}$ was determined.

(2) The correlation between the dynamic behavior of VERP, APD, and STI after the abrupt PCL change $800-350 \mathrm{msec}$ before and after d-sotalol treatment was determined. Furthermore, the correlation between APD and STI after the abrupt PCL change $1000-500 \mathrm{msec}$ was determined.

(3) The fit characteristics of the bi-exponential model for the dynamic behavior of VERP, APD, and STI after the abrupt PCL decrease $800-350 \mathrm{msec}$ and for STI also after the abrupt PCL decrease $1250-350$ msec were computed.

\section{d-Sotalol}

The dose of d-sotalol given, was guided by the response at $800 \mathrm{msec}$ PCL. If the increase in STI, VERP, and APD did not exceed the control value by $10 \%$ the dose was increased. This led to a dose ranging from $2-3 \mathrm{mg} / \mathrm{kg}$ in 5 minutes.

\section{Electrical Recordings and Pacing}

Monophasic action potentials were recorded from the right ventricular endocardium close to the pacemaker electrode. The monophasic action-potential catheter (Franz MAP-Pacing catheter, EP Technologies Inc., Mountain View, CA, USA) was introduced via the jugular or femoral vein and positioned with the help of fluoroscopy. The monophasic action potential was amplified by a direct current coupled amplifier, which has a $20 \mathrm{mV}$ calibration pulse. The minimum amplitude for acceptance of the signal was $15 \mathrm{mV} .11$

The dogs were continuously paced unipolarly at twice the diastolic threshold with a custom built stimulator (Vitatron Medical B.V., Dieren, The Netherlands) which allowed monitoring and recording of the endocardiallyevoked response. Furthermore this stimulator generated a stimulation marker, an $\mathrm{R}$-wave marker (indicating the moment of R-wave sensing from the endocardial signal), and a T-wave marker (indicating the moment of T-wave sensing from the endocardially-evoked $\mathrm{T}$-wave). Sensitivity for $\mathrm{R}$-wave and $\mathrm{T}$-wave sensing was adjusted according to the measured amplitucles.

\section{Pacing Protocol}

To determine steady-state values and to explore the dynamic behavior of VERP, APD, and STI after a sudden decrease in PCL, four protocol components (A, $B, C$, and D) were used (Figure 5.1) In this example we use 800 and $350 \mathrm{msec}$ PCL. Except for the PCLs used, the protocol components are identical for the other PCLs applied.

Protocol Component A - 800 msec steady-state: After 5 minutes of continuous pacing at $800 \mathrm{msec}$ PCL steady-state values of APD and STI were registered. VERP was determined by incremental scanning with $5 \mathrm{msec}$ increments and without an intertrain pause, 1,24,25 

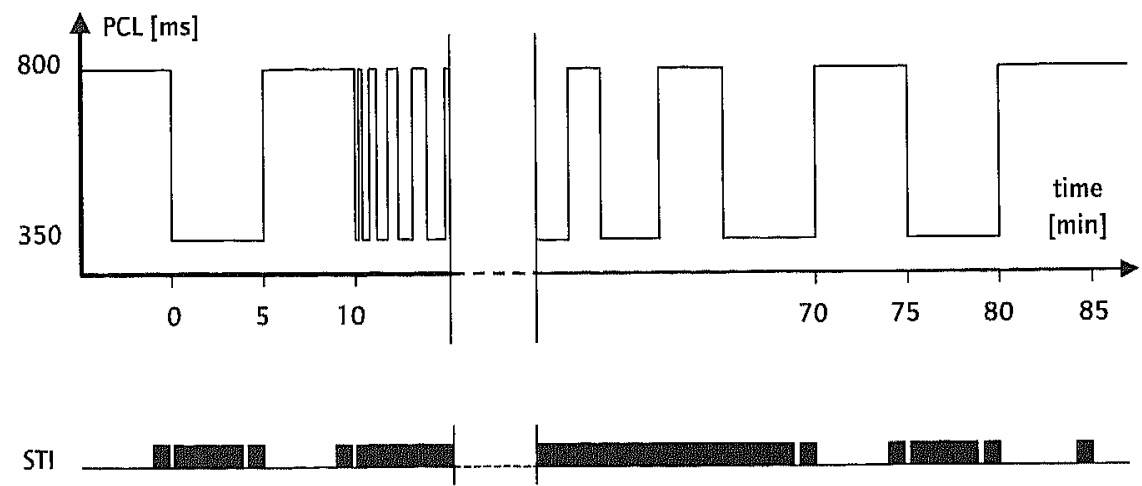

[62]

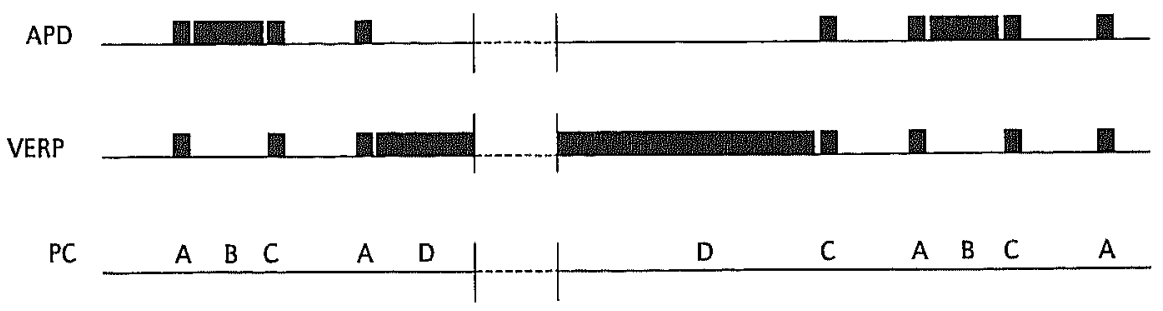

Figure 5.1 - Schematic representation of the pacing protocol.

The upper part of the figure shows how the $\mathrm{PCL}$ changes (ordinate) in time (abscissa), during the course of the experiment. In the lower part the filled bars indicate when the value of a variable (STI, APD or VERP) is determined. The short bars indicate momentary measurements, whereas the long bars indicate continuous measurements during adaptation from 800 to $350 \mathrm{msec}$ steady-state. These measurements are also indicated as PCs to demonstrate the sequence and repetition of these PCs (see also Methods: Pacing Protocol), Steady-state values of VERP, APD, and STI are determined after 5 minutes of continuous pacing at 800 (PC-A; $t=0,10,75$, 85 minutes) and $350 \mathrm{msec} P C \mathrm{PC}$ ( $\mathrm{PC}-\mathrm{C} ; \mathrm{t}=5,70,80$ minutes). To study the dynamics of STI and APD the pacing cycle length is abruptly decreased from 800 to $350 \mathrm{msec}$ ( $P C \cdot B ; t=0 \cdot 5$ minutes and $t=75-80$ minutes). To study the dynamics of VERP, multiple rate changes are needed (PC-D; $t=10-70$ minutes). Abbreviations: $\mathrm{PCL}=$ pacing cycle length; $\mathrm{ST} I=$ stimulus-T interval; $\mathrm{APD}=$ action-potential duration; $\mathrm{VERP}=$ ventricular effective refractory period; $\mathrm{PC}=$ protocol components.

Protocol Component B - dynamics of APD and STI: Starting at $800 \mathrm{msec}$ steady-state pacing, the PCL was abruptly decreased to $350 \mathrm{msec}$. Continuous pacing at $350 \mathrm{msec}$ PCL was maintained for at least 5 minutes. During this period all surface leads were recorded continuously.

Protocol Component $C$ - $350 \mathrm{msec}$ steady-state: After 5 minutes of continuous pacing at $350 \mathrm{msec}$ PCL, STI and APD were registered and VERP was determined.

Protocol Component D - dynamics of VERP: Because initial VERP shortening was much more pronounced compared to the subsequent phase the dynamics of VERP were determined using two subprotocols. 
First phase ( $0-100$ beats): After a certain number of beats $(1,3,5, \ldots$, $17,20,25,35,45,55,75,100$ ) with intervals of $350 \mathrm{msec}$ following steadystate pacing with a PCL of $800 \mathrm{msec}$, a premature test pulse, with an initial coupling interval shorter than VERP, was given. The heart was then brought back to $800 \mathrm{msec}$ steady-state followed by the same $350 \mathrm{msec}$ drive train with a $5 \mathrm{msec}$ incremented coupling interval for the premature beat. This procedure was repeated until capture occurred. ${ }^{1}$

Second phase ( $>100$ beats): In the continuous pacing train (SI-SI) of $350 \mathrm{msec}$ PCL a test pulse S2 was introduced after every fourth beat S1. The initial S1S2 interval was chosen to be $5 \mathrm{msec}$ shorter than the VERP found after 100 beats. Because VERP continues to shorten S2 will eventually capture the ventricle. The time from the onset of the $350 \mathrm{msec}$ pacing train to the last noncapturing 52 indicates the moment VERP is longer than the $\$ 152$ coupling interval. The coupling interval for the test stimulus was subsequently decremented by $5 \mathrm{msec}$ and the procedure was repeated after the heart was brought back to $800 \mathrm{msec}$ steady-state. The shortest coupling interval for the test stimulus tested was equal to the steady-state VERP at 350 msec PCL. 1

\section{Data Acquisition}

All signals and markers were sampled at a rate of $1 \mathrm{kHz}$ and stored on a hard disk, together with 6 surface ECG leads.

\section{AV Relation}

All experiments were performed in dogs with AV block using right ventricular endocardial pacing with a free running atrial rhythm. To study the influence of timing of the atrial contraction on the duration of ventricular repolarization the peak P-wave (lead II) to stimulus interval and the subsequent STI as a representative parameter of the duration of ventricular repolarization was determined at 800 and $350 \mathrm{msec}$ PCL in six dogs. The P-stimulus interval and STI was determined in 20 cycles. If no P-wave was visible in lead II the cycle was skipped.

Data Analysis and Statistics

APD and STI were determined via off-line analysis. VERP, APD, and STI were determined with $5 \mathrm{msec}$ precision.

Statistical significance of d-sotalol treatment was assessed by a paired $t$-test. ANOVA followed by Bonferroni $t$-tests was used to compare measured values and values computed by the bi-exponential model and the amount of shortening during this first exponential component $\left(c_{1}\right)$ with the second component $\left(c_{2}\right)$. The same test was used to compare the total amount of shortening $(\triangle)$ of VERP, APD, and STI for the PCL decrease 1000 - 500 with 800 - 350 msec. The dynamics of VERP, APD, and STI were compared using linear regression analysis from beat 0 150. The endpoint for the regression analysis was chosen at beat 150 because 
steady-state appeared to be reached by then. For STI, statistical significant difference comparing the parameters of the bi-exponential model for the PCL decrease $800-350 \mathrm{msec}$ with the PCL decrease $1250-350 \mathrm{msec}$ was tested using a paired $t$-test.

The software used to fit the bi-exponential model was SPSS for Windows. The software used for the statistical tests was Primer of Biostatistics (McGraw-Hill Inc., New York, NY, USA). P values $<0.05$ were considered significant.

Bi-Exponential Model

Although linear regression analysis can be used to quantify the similarity of VERP, APD, and STI dynamics, this technique can not show how the characteristics of repolarization dynamics change applying different protocols. Therefore a

[64] bi-exponential model $y=c_{0}+c_{1} \exp \left(-t / \tau_{1}\right)+c_{2} \exp \left(-t / \tau_{2}\right)$ was used.

In this model y represents VERP, APD, or STI, t represents time expressed in beats of $350 \mathrm{msec}$ PCL (e.g. the value of VERP, APD, or STI $3.5 \mathrm{sec}$ after the change in PCL will be found for $t=10$ ).

A few characteristic values for $t$ will be used to illustrate the behavior of the bi-exponential model:

$$
y=c_{0}+c_{1} \exp \left(-t / \tau_{1}\right)+c_{2} \exp \left(-t / \tau_{2}\right)
$$

The steady-state value for VERP, APD or STI before the change in PCL will be found at $\mathrm{t}=0 . \mathrm{t}=0$ indicates 0 beats after of the short PCL.

For $\mathrm{t}=0$ Equation ( 1 ) will become:

$$
\begin{aligned}
& y=c_{0}+c_{1} \exp \left(-0 \mathrm{~d} / \tau_{1}\right)+c_{2} \exp \left(-0 / \tau_{2}\right) \\
& y=c_{0}+c_{1} \exp (0)+c_{2} \exp (0) \\
& \text { since } \exp (0)=1 \text { this will become } \\
& y=c_{0}+c_{1}+c_{2}
\end{aligned}
$$

This is the steady-state value for VERP, APD, or STI before the change in PCL. The steady-state value for VERP, APD, or STI after the change in PCL will be found after a few minutes pacing at the short PCL. Pacing with a PCL of $350 \mathrm{msec}$, this could correspond to approximately 500 beats of $350 \mathrm{msec}$, therefore $t=500$.

For $\mathrm{t}=500$ Equation (1) will become:

$$
y=c_{0}+c_{1} \exp \left(-500 / \tau_{1}\right)+c_{2} \exp \left(-500 / \tau_{2}\right)
$$


If $\tau_{1}$ and $\tau_{2} \ll 500: \exp \left(-500 / \tau_{1}\right) \approx 0$ and $\exp \left(-500 / \tau_{2}\right) \approx 0$. Therefore:

$$
\mathrm{y}=\mathrm{c}_{0}
$$

This is the steady-state value for VERP, APD, or STI at the short PCL.

If $\tau_{1} \ll \tau_{2}$, the change in $y$ during the first beats is manly determined by the first exponential component $\left(c_{1} \exp \left(-t / \tau_{1}\right)\right)$. The value of the first exponential component already shows considerable change, while the second component $\left(c_{2} \exp \left(-t / \tau_{2}\right)\right)$ shows hardly any change. Hence, a small time constant corresponds to an exponential component which rapidly diminishes. This behavior can be illustrated for $t=\tau_{1}$.

For $\mathrm{t}=\tau_{1}$ Equation ( 1 ) will become:

$$
\begin{aligned}
& y=c_{0}+c_{1} \exp \left(-\tau_{1} / \tau_{1}\right)+c_{2} \exp \left(-\tau_{1} / \tau_{2}\right) \\
& \text { since } \tau_{1}<<\tau_{2}, c_{2} \exp \left(-\tau_{1} / \tau_{2}\right) \approx c_{2} . \text { Therefore: } \\
& y=c_{0}+c_{1} / 2.7+c_{2} \\
& y=c_{0}+0.37 c_{1}+c_{2}
\end{aligned}
$$

If $\tau_{1} \ll \tau_{2}$, the change in $y$ after the first beats is mainly determined by the second exponential component $\left(c_{2} \exp \left(-t / \tau_{2}\right)\right)$. The value of the first exponential component is almost 0 , while the second component still plays an important role. This behavior can be illustrated for $\mathrm{t}=\tau_{2}$.

$$
\begin{aligned}
& \text { For } t=\tau_{2} \text { Equation (1) will become: } \\
& \begin{array}{l}
y=c_{0}+c_{1} \exp \left(-\tau_{2} / \tau_{1}\right)+c_{2} \exp \left(-\tau_{2} / \tau_{2}\right) \\
\text { since } \tau_{1}<<\tau_{2}, c_{1} \exp \left(-\tau_{1} / \tau_{2}\right) \approx 0 \text {. Therefore: } \\
y=c_{0}+c_{2} / 2.7 \\
y=c_{0}+0.37 c_{2}
\end{array}
\end{aligned}
$$

The change in VERP, APD or STI $(\Delta y)$ due to the change in PCL is the difference between the value at the long PCL (Equation (2): $y=c_{0}+c_{1}+c_{2}$ ) and the value at the short PCL (Equation (3): $y=c_{0}$ ). Therefore:

$$
\begin{aligned}
& \Delta y=\left(c_{0}+c_{1}+c_{2}\right)-\left(c_{0}\right) \\
& \Delta y=c_{1}+c_{2}
\end{aligned}
$$


Table 5.1 Steady state values of VERP, APD and STI.

\begin{tabular}{|c|c|c|c|c|c|c|c|}
\hline & & \multicolumn{6}{|l|}{$\mathrm{PCl}$} \\
\hline & & \multirow[t]{2}{*}{1000} & \multicolumn{2}{|l|}{800} & \multirow[t]{2}{*}{500} & \multicolumn{2}{|l|}{350} \\
\hline & & & control & d-sotalol & & control & $d-s$ \\
\hline \multirow[t]{3}{*}{ VERP } & $n$ & 6 & 10 & 7 & 6 & 10 & 7 \\
\hline & mean $\pm S D$ & $252 \pm 14$ & $230 \pm 15$ & $257 \pm 24^{*}$ & $202 \pm 9$ & $170 \pm 12$ & 18 \\
\hline & range & $240-275$ & $210-255$ & $215-290$ & $190-215$ & $150-185$ & 17 \\
\hline \multirow[t]{3}{*}{ APD } & $n$ & 6 & 8 & 6 & 6 & 8 & 6 \\
\hline & mean $\pm S D$ & $295 \pm 20$ & $273 \pm 21$ & $297 \pm 28^{*}$ & $239 \pm 13$ & $208 \pm 19$ & 21. \\
\hline & range & $264-316$ & $240-290$ & $255-330$ & $224 \cdot 256$ & $175 \cdot 220$ & 78 \\
\hline \multirow[t]{3}{*}{ STI } & $\mathrm{n}$ & 6 & 10 & 7 & 6 & 10 & 7 \\
\hline & mean \pm 50 & $307 \pm 13$ & $281 \pm 13$ & $319 \pm 32^{*}$ & $247 \pm 8$ & $211 \pm 14$ & 22 \\
\hline & range & $288-324$ & $260-300$ & $260-360$ & $236-256$ & $190 \cdot 230$ & 20 \\
\hline
\end{tabular}

Abbreviations; $\mathrm{VERP}=$ ventricular effective refractory period; $\mathrm{APD}=$ action-potential duration; $\mathrm{STI}=$ $T$ interval; $\mathrm{PCL}=$ pacing cycle length; $n=$ number of dogs. Significant difference between control an sotalol values at 800 and $350 \mathrm{msec} P C L$ is indicated by * (paired ttest, $P<0.05$ ). All values in mse for $n$.

Results

The cycle length of the idioventricular rate of the anesthetized d, determined before the pacing protocol started. The cycle length ranged fr- $2500 \mathrm{msec}$ with a mean of $1500 \pm 500 \mathrm{msec}$.

\section{Steady-State}

In Table 5.1, steady-state values at $1000,800,500$, and $350 \mathrm{msec} \mathbf{F}$ VERP, APD, and STI are shown. Each parameter displays a normal fre dependency: PCL decrease leads to shortening (Table 5.1). In the same $\mathrm{C}$ is always longer than APD and APD is always longer than VERP irrespe $\mathrm{PCL}$ and conditions. This could be the consequence of the parameters' de (sce Discussion).

For the PCLs 800 and $350 \mathrm{msec}$ the parameters are presented bef after d-sotalol administration. Since the effect of $d$-sotalol has been demol to be reverse use dependent, lengthening at $800 \mathrm{msec} P C L$ is more outspols at 350 msec PCL (Table 5.1) leading to a significant increase in $\triangle$ for VE STI (Table 5.2). Although the PCL decrease $800-350 \mathrm{msec}$ is less than 1 decrease $1000-500 \mathrm{msec}, \triangle V E R P$ and $\triangle S T I$ is significantly longer for $\mathrm{t}$ reduction $800-350 \mathrm{msec}$. This becomes even more pronounced after d administration leading to a significant increase for $\triangle V E R P, \triangle A P D$, and $\triangle S$ 
Table 5.2 Shortening of VERP, APD and STI after an abrupt PCL decrease.

\begin{tabular}{|c|c|c|c|c|}
\hline & & \multicolumn{3}{|l|}{ PCL decrease } \\
\hline & & \multirow[t]{2}{*}{$1000-500$} & \multicolumn{2}{|l|}{$800-350$} \\
\hline & & & control & d-sotalol \\
\hline \multirow[t]{2}{*}{$\triangle V E R P$} & $n$ & 6 & 10 & 7 \\
\hline & mean $\pm S D$ & $50 \pm 10$ & $60 \pm 8 \#$ & $72 \pm 15^{* \#}$ \\
\hline \multirow[t]{2}{*}{$\triangle \mathrm{APD}$} & $n$ & 6 & 8 & 6 \\
\hline & mean $\pm S D$ & $56 \pm 11$ & $64 \pm 8$ & $83 \pm 11 \#$ \\
\hline \multirow[t]{2}{*}{$\Delta S T I$} & $n$ & 6 & 10 & 7 \\
\hline & mean $\pm S D$ & $60 \pm 9$ & $71 \pm 6$ & $91 \pm 19$ * \# \\
\hline
\end{tabular}

Abbreviations: VERP $=$ ventricular effective refractory period; $\triangle V E R P=$ amount of VERP shortening; $A P D=$ action-potential duration; $\triangle \mathrm{APD}=$ amount of $\mathrm{APD}$ shortening; $S T$ I $=$ stimulus- $\mathrm{T}$ interval; $\triangle \mathrm{STI}=$ amount of STl shortening; $P C L=$ pacing cycle length; $n=$ number of dogs, Significant difference between control and $d$-sotalol values for the $P C L$ decrease $800-350 \mathrm{msec}$ is indicated by * (paired ttest, $P<0.05$ ). Significant difference for $\triangle V E R P, \triangle A P D$ and $\triangle S T I$ comparing the $P C L$ decrease $1000-500$ and $800-350 \mathrm{msec}$ is indicated by \# (ANOVA followed by Bonferroni $t$-tests, $p<0.05$ ). All values in msec, except for $n$.

Standard deviations and ranges of steady-state values of VERP, APD, and STI indicate a large interindividual variation (Table 5.1). Linear regression analysis shows that this variation is not related to the prepacing values of the idio-ventricular rate during anesthesia $\left(\mathrm{r}^{2}<0.24, \mathrm{P}>0.15\right)$. In other words, the dogs with the, respectively, slower or faster idioventricular rate during anesthesia do not necessarily have the longer, or shorter, value for VERP, APD, and STI.

\section{Dynamics}

Applying linear regression analysis to compare the dynamics of VERP, APD, and STI for the same dog, using all data points from the onset of the simulated VT until beat 150 , high values for $\mathrm{r}^{2}$ were found for the PCL clecrease $800-350 \mathrm{msec}$ $\left(r^{2} \geq 0.94\right)$, the PCL decrease $800-350$ after d-sotalol treatment $\left(r^{2} \geq 0.93\right)$, and the $\mathrm{PCL}$ decrease $1000-500 \mathrm{msec}\left(\mathrm{r}^{2}=0.95\right)$. This indicates that although the start and endpoints for the three variables can be different and their respective amount of shortening (Tables 5.1 and 5.2), the dynamics of the repolarization parameters show the same behavior.

\section{Bi-Exponential Model}

Table 5.3 shows the results of the non-linear regression analysis for the biexponential model for VERP, APD, and STI for the PCL decrease from 800 to 350 msec and for STI for the PCL decrease from 1250 to $350 \mathrm{msec}$. Figure 5.2 is a graphic representation of the results for the PCL decrease from 800 to $350 \mathrm{msec}$. 
Table 5.3 Fitting the bi-exponential model: $y=c_{0}+c_{1} \exp \left(-t / \tau_{1}\right)+c_{2} \exp \left(-t / \tau_{2}\right)$.

\begin{tabular}{|c|c|c|c|c|c|c|c|c|c|}
\hline & $\begin{array}{l}\text { PCL decrease } \\
\text { (msec) }\end{array}$ & $\begin{array}{l}c_{0}+c_{1}+c_{2} \\
\text { (msec) }\end{array}$ & $\begin{array}{l}c_{0} \\
\text { (msec) }\end{array}$ & $\begin{array}{l}c_{1}+c_{2}(\Delta) \\
\text { (msec) }\end{array}$ & $\begin{array}{l}\tau_{1} \\
\text { (beats) }\end{array}$ & $\begin{array}{l}c_{1} \\
\text { (msec) }\end{array}$ & $\begin{array}{l}\tau_{2} \\
\text { (beats) }\end{array}$ & $\begin{array}{l}c_{2} \\
\text { (msec) }\end{array}$ & $r^{2}$ \\
\hline $\begin{array}{l}\text { VERP } \\
n=10\end{array}$ & $800-350$ & $226 \pm 15$ & $171 \pm 14$ & $55 \pm 9$ & $1.3 \pm 1.1$ & $17 \pm 7$ & $63 \pm 17$ & $38 \pm 5 \#$ & $\begin{array}{l}0.99 \pm \\
0.01\end{array}$ \\
\hline $\begin{array}{l}\text { APD } \\
n=8\end{array}$ & $800 \cdot 350$ & $257 \pm 20$ & $203 \pm 16$ & $64 \pm 6$ & $0.9 \pm 0.9$ & $25 \pm 6$ & $47 \pm 8$ & $39 \pm 8 \#$ & $\begin{array}{l}0.98 \pm \\
0.01\end{array}$ \\
\hline $\begin{array}{l}\text { STI } \\
n=10\end{array}$ & $800-350$ & $281 \pm 13$ & $212 \pm 14$ & $69 \pm 6$ & $0.4 \pm 0.3$ & $23 \pm 2$ & $45 \pm 9$ & $45 \pm 9 \#$ & $\begin{array}{l}0.99 \pm \\
0.01\end{array}$ \\
\hline $\begin{array}{l}S T I \\
n=5\end{array}$ & $1250-350$ & $327 \pm 32^{*}$ & $218 \pm 12$ & $109 \pm 25^{*}$ & $0.4 \pm 0.4$ & $39 \pm 79^{*}$ & $46 \pm 10$ & $70 \pm 9 * \#$ & $\begin{array}{l}0.99 \pm \\
0.01\end{array}$ \\
\hline
\end{tabular}

[68 ] Abbreviations: VERP $=$ ventricular effective refractory period; $A P D=$ action-potential duration; $S T$ sT $=$ stimulus-T interval; $P C L=$ pacing cycle length; $n=$ number of dogs. Bi-exponential curve fitting: $y=c_{0}+c_{1} \exp \left(-t / \tau_{1}\right)+c_{2} \exp$ $\left(-t / \tau_{2}\right)$. y Represents VERP, APD, or STI; $t$ represents time, expressed in beats with $350 \mathrm{msec} P C L$. The table shows steady-state values of VERP, APD, and STl at the long PCL $\left(c_{0}+c_{1}+c_{2}\right)$ and the short PCL $\left(c_{0}\right)$. The total amount of shortening after the abrupt PCl. decrease $800-350$ msec and $1250-350$ msec is indicated by $c_{1}+c_{2}(\Delta), \tau_{1}$ and $\tau_{2}$ are the time constants of the first respectively second exponential phase, expressed in beats with $350 \mathrm{msec} \mathrm{PCL}$ For STI, significant difference between the values for the PCL decrease $1250-350 \mathrm{msec}$ and $800-350 \mathrm{msec}$ is indicated by * (paired ttest, $P<0.05$ ). Significant difference between $c_{1}$ and $c_{2}$ is indicated by \# (ANOVA followed by Bonferroni $t$ tests, $P<0.05$ ). All values are expressed as mean $\pm S D$. For further explanation see also "Bi-exponential Model" in Methods and Discussion section.

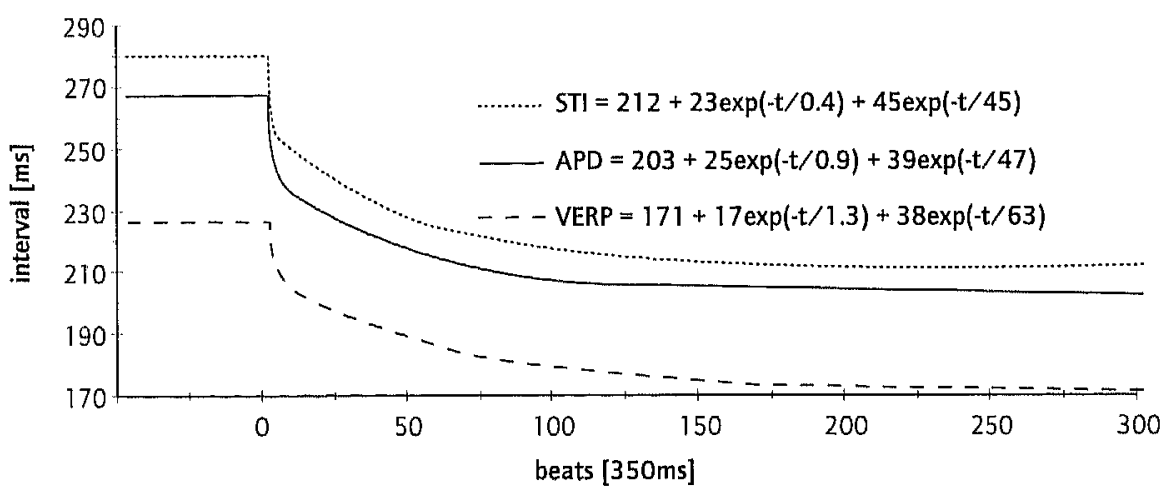

Figure 5.2 - Graphic representation of the results found after non-linear regression analysis using the biexponential model for the PCL decrease from 800 to $350 \mathrm{msec}$ (see Table 5.3).

The ordinate represents VERP, APD, and STI. On the abscissa, time is expressed in number of beats with a 350 msec PCL. The curve starts with a plateau phase expressing steady-state at $800 \mathrm{msec} P C L$. The moment the PCL is abruptly decreased to $350 \mathrm{msec}$, all three variables shorten. Initial shortening is very pronounced, thereafter, shortening gradually decreases. Finally, there is a asymptotic approach to steady-state values at $350 \mathrm{msec} P C L$. Abbreviations: $P C L=$ pacing cycle length; $V E R P=$ ventricular effective refractory period; $A P D=$ action-potential duration; $S T \mathrm{Tl}=$ stimulus $-\mathrm{T}$ interval. 
The values computed by the model for VERP, APD, and STI at $800 \mathrm{msec}$ PCL, 350 msec PCL and $\Delta$ showed no significant difference compared to the matching values in Tables 5.1 and 5.2.

For the PCL decrease from 800 to $350 \mathrm{msec}$ and from 1250 to $350 \mathrm{msec}$, the value of parameter $\tau_{1}$ indicated a very short first phase of shortening immediately after the onset of the simulated VT, $\tau_{2}$ was much longer. This means that shortening in the second exponential component, which starts after approximately three times $\tau_{1}$, is much slower compared to the first exponential component. The amount of shortening during this second exponential component $\left(\mathrm{c}_{2}\right)$ is significantly more than during the first component $\left(c_{1}\right)$.

As expected, the computed steady-state value for STI at $1250 \mathrm{msec}$ is significantly longer than the steady-state value at $800 \mathrm{msec}$ (Table 5.3). Since there is no statistical difference between the steady-state value at $350 \mathrm{msec}$ for the PCL decrease from 1250 to $350 \mathrm{msec}$ compared to the PCL decrease from 800 to 350 msec this leads to a significant increase in the total amount of shortening $\Delta\left(c_{1}+\right.$ $\left.c_{2}\right)$. This increase is accounted for by a significant increase in the amplitude of both the first $\left(c_{1}\right)$ and second $\left(c_{2}\right)$ exponential phase without a significant change in $\tau_{1}$ and $\tau_{2}$.

\section{Effect of AV Relation}

In six dogs, tested during steady-state pacing with 800 or 350 msec PCL, STI is almost constant showing a maximum fluctuation of $8 \mathrm{msec}$. On the other hand, the interval between spontaneous atrial activation and ventricular pacing (P-stimulus interval) randomly covers the ranges from 0 to $336 \mathrm{msec}$ for $350 \mathrm{msec}$ PCL and from 24 to $696 \mathrm{msec}$ for $800 \mathrm{msec}$ PCL.

\section{Discussion}

Under different circumstances we determined and compared steady-state values and dynamics of VERP, APD, and STI. All three parameters showed a normal frequency dependency and increased significantly after d-sotalol administration. Standard deviations of steady-state and dynamic values indicated a considerable interindividual variation. However, the dynamics of VERP, APD, and STI after an abrupt decrease in PCL were highly correlated. The best mathematical model to describe these dynamics was a bi-exponential model with a very short first and a much longer second time constant.

The observed variation between dogs indicates that prediction of steadystate and dynamic values of repolarization and refractoriness, especially at the onset of VT, seems only feasible with individual on-line assessment as can be provided by STI. 
Steady-State

In the same individual, STI is always longer than APD and APD is always longer than VERP irrespective of PCL and conditions (Table 5.1). This is determined by the way these parameters are defined. STI will be the longest in duration because STI measures not only repolarization but also conduction. APDI00 is a measure for the total duration of repolarization and will be longer than VERP because refractoriness ends before repolarization.

Before steady-state values of VERP, APD, and STI were determined, pacing at the PCL tested was continued for at least 3 minutes. Steady-state values of VERP were determined with premature electrical stimulation, using incremental scanning without intertrain pauses to preserve steady-state during the scanning process. ${ }^{1,24,25}$ Since there is no compensatory lengthening of VERP due to the drop from paced rate to spontaneous rate during the intertrain pause, shorter values for VERP can be found compared to protocols with intertrain pauses. On the other hand reproducibility will increase, 6,24 Steady-state values of APD and STI can be determined without the need of premature electrical stimulation.

Although the scanning protocol guarantees maximum reproducibility, steady-state values, for example, at $800 \mathrm{msec}$ PCL of VERP, APD, and STI in different dogs show variations as high as 45,50 , and $40 \mathrm{msec}$, respectively (Table 5.1). These variations were also found by others, not only for VERP 1,6 but also for $\mathrm{APD}^{26}$ and STI. ${ }^{27}$ The reason for this variation is not clear since prepacing idioventricular rate or the lack of AV synchronization do not seem to be contributing factors. Since we did not control the adrenergic tone the relevance of variation in adrenergic tone for this variation could not be determined. In previous work we demonstrated similar variations for VERP even in the same dog in subsequent experiments. 28

\section{Dynamics}

Steady-state values found for VERP, APD, and STI within an individual animal under identical circumstances were not identical. This results in differences for the total amount of shortening for these parameters after an abrupt PCL decrease (Table 5.2). Nevertheless, the adaptation of VERP, APD, and STI after an abrupt PCL decrease was highly correlated. This correlation changed neither for different PCL decreases nor after the administration of d-sotalol.

The dynamics of repolarization and refractoriness, after an abrupt decrease in PCL, have been previously studied. Generally, in these studies a single variable was investigated, for example VERP1,2,6-8 or APD., ${ }^{4-9-13}$ The correlation between VERP and APD has been studied in six patients in the dynamic situation alter a step increase in drive frequency. ${ }^{18}$ Although temporal resolution of the VERP determination was limited the authors showed that VERP and APD shortening after a step increase in pacing frequency of $49.3 \%$ were nearly identical: $18.12 \%$ VERP shortening and $17.43 \%$ APD shortening.

During the experiments we did not control autonomic tone. Nevertheless, 
STI and APD values were reproducible in time (Figure 5.1). Since STI not only represents repolarization but also conduction properties, STI can be more strongly influenced by autonomic tone than VERP or APD. However, very high correlations between STI and VERP or APD were found. These correlations did not change for the different PCL changes that were applied in the absence or presence of d-sotalol.

\section{Spatial and Temporal Resolution}

In this study the duration of ventricular repolarization was determined by simultaneous endocardial measurements of VERP, APD, and STI from one site in the right ventricular endocardium. An important property all three parameters share is that they can be determined in the clinical setting. STI can even be made available on-line in permanent pacemakers. ${ }^{5}$ Since VERP and STI determination require electrical stimulation of the heart, sinultaneous determination at multiple sites, to study spatial distribution is not feasible. On the other hand, it is feasible to conduct simultaneous APD recordings at multiple sites using monophasic action-potential catheters. However, maximal spatial resolution is low due to the limited amount of monophasic action-potential catheters that can be positioned simultaneously. Other parameters to monitor repolarization simultaneously at multiple sites such as ARI ${ }^{15,16}$ have not been applied in the clinical setting. Optical mapping, using voltage sensitive dyes, offers both high temporal and spatial resolution, however this method can only be applied in isolated hearts. ${ }^{13,29,30}$

\section{Bi-Exponential Model}

A mathematical model can be a helpful tool to describe the behavior of a certain parameter in time. A good model is often a difficult trade between simplicity of the model and the quality of the fit. In other words, a simple mathematical model is easy to handle but the values predicted by the model might show some systematic difference compared to the measured values. On the other hand, reduction of this difference (residue) could lead to unnecessarily complicated models.

Analysis of the behavior of STI, VERP, and APD shows dramatic shortening immediately after the abrupt decrease in cycle length. The shortening is less pronounced in the subsequent beats and, finally, there is an asymptotic approach towards the new steady-state value. This behavior seems to be a typical exponential response: a fast initial response followed by a slower response and finally a asymptotic approach, and could be described by a mono-exponential model ( $\mathrm{y}=$ $\left.c_{0}+c_{1} \exp \left(-t / \tau_{1}\right)\right)$. We found excellent fit statistics $\left(r^{2} \geq 0.90\right)$ fitting this model. However, the mono-exponential model showed systematic residual variation. The model underestimated the shortening in the first 25 beats and overestimates shortening in the latter part. Adding a second exponent (bi-exponential model: $y=c_{0}$ $\left.+c_{1} \exp \left(-t / \tau_{1}\right)+c_{2} \exp \left(-t / \tau_{2}\right)\right)$ to the model the shortening behavior is split in two parts: one in which the first exponent plays the most important role and one in 
which the second exponent plays the most important role. The mathematical model is more complex but the model will fit the data during all shortening phases. This resulted in an even better coefficient of determination $\left(r^{2} \geq 0.99\right)$ compared to the mono-exponential model. However, the most important reason to use the bi-exponential model was the absence of systematic residual variation.

Other investigators preferred the mono-exponential model. ${ }^{12-14}$ Most probably they did not find a systematic residual variation. This can be due to the limited time resolution of the data obtained by these authors. The pronounced difference in the amount of shortening in the first few beats compared to the following beats can only be demonstrated to its full extent if the variables are studied on a beat-to-beat basis. Furthermore, the interval taken to fit the model plays an important role. The longer the interval after the change in $\mathrm{PCL}$, the more data in

[72 ] the fit correspond to the second phase which is mono-exponential. This will lead to a false improvement of fit parameters of the mono-exponential model. Applying the bi-exponential model starting at the moment of the abrupt decrease in PCL and ending three minutes later, this model fits the repolarization dynamics as assessed by VERP, APD, and STI without systematic residual variations and excellent fit statistics.

Since the first time constant $\tau_{1}$ is shorter than one beat the quality of $\tau_{1}$ has its limitations. The time constant of the second exponential phase $\tau_{2}$ enables calculation of the time steady-state at the short PCL will be reached. After three times $\tau_{2}$ only $5 \%$ of the initial amplitude $c_{2}$ remains. Since $\tau_{1}$ is much smaller than $\tau_{2}$, the contribution of the first component can be neglected.

Oscillations in Repolarization Dynamics

In our experiments the dynamics of VERP, APD, and STI never showed oscillations in response to the abrupt decrease in PCL. However, in the literature oscillations in shortening of VERP1,31 and APD 10,12,13 after an abrupt decrease in PCL have been described. Although the reason for oscillation remains unclear it seems related to the amount of change in PCL: as the short PCL approaches the VERP of the long PCL, the greater the chance for oscillations to occur. For the PCL decrease from 800 to $350 \mathrm{msec}$ after d-sotalol treatment, VERP at $800 \mathrm{msec}$ PCL was $257 \pm 24 \mathrm{msec}$, which is much shorter than the short PCL ( $350 \mathrm{msec}$ ). For the same PCL decrease before d-sotalol treatment or the PCL decrease from 1000 to $500 \mathrm{msec}$ the difference between the short PCL and the VERP of the long $\mathrm{PCL}$ is even bigger.

\section{Complete AV Block}

The hearts of the dogs used in our experiments had induced chronic AV block that resulted in biventricular hypertrophy due to volume overload. 32 Since hypertrophy is known to lengthen repolarization parameters, this could be a confounding parameter, not only for steady-state values but also for the dynamic response to the change in PCL. Denardo and Stevenson ${ }^{33}$ compared the dynamics 
of VERP in patients with structurally normal hearts to patients with hypertrophy with or without chronic heart failure. They found that although VERP was lengthened, the ability of VERP to adlapt to a sudden increase in heart rate was not impaired in the latter groups.

\section{Applications of Stimulus-T Interval Information}

Since STl can be determined in an implantable pacing system with a normal pacemaker lead, several applications of this potential have been described. It is well known as a physiological sensor for rate responsive pacing. ${ }^{5}$ Furthermore, it has been described as an indicator for drug-induced changes in patients, ${ }^{34}$ an early indicator for subendocardial ischemia, ${ }^{35}$ a differentiator between ventricular tachycardia and sinus tachycardia, ${ }^{36}$ and as a system for automatic capture recognition. ${ }^{37}$ Our group demonstrated a high correlation between VERP, APD, and STI in the dynamic situation after an abrupt decrease in PCL. One of the major findings is that, although experimental circumstances are identical, steady-state values of VERP, APD, and STI show a pronounced variation between individuals. This might be relevant for ATP, since the coupling interval of premature stimuli should approximate VERP, which is, especially in the dynamic situation after the onset of VT, hard to predict. On-line feedback with regard to the situation of repolarization, as can be provided by STI, could be used to generate a dynamic and individually suited ATP mode in which coupling intervals could be selected using individual and on-line available STI information.

\section{Limitations of the Study}

One of the prerequisites of STI measurement is an endocardially-evoked response, in other words STI can only be determined after pacing. This might be considered a shortcoming of this technique, since pacing will interrupt spontaneous activation. However pacing from a certain site will generate a reproducible depolarization- and repolarization front in the vicinity of the pacing lead. As a result of that STI will be very reproducible. Furthermore, STI can be determined with a normal, chronically stable, pacemaker lead.

In this study VERP, APD, and STI behavior was not assessed in ischemic tissue. In these circumstances VERP can continue after repolarization has completed (post repolarization refractoriness). As a consequence the VERP-APD relation will change. The question which then rises is towards the behavior of STI.

\section{Conclusion}

After an abrupt decrease in PCL, as occurs during VT, the duration of repolarization and refractoriness will decrease. In this dynamic situation the behavior of VERP, APD, and STI is highly correlated and best represented by a bi-exponential model. The observed variation between individuals indicates that prediction 
of the duration of repolarization and refractoriness by previous determination in other individuals may not be accurate and has to be replaced by on-line assessment as can be provided by STI. An ATP mode in which timing of premature stimuli is guided by on-line STI information seems feasible.

Acknowledgments: We are grateful to Mr. J. Verschuuren, Bristol Meyers Squibb, Brussels, Belgium who provided us with d-sotalol

\section{References}

1. Janse MJ, van der Steen ABM, van Dam RT, et al. Refractory period of the dog's ventricular myocardium following sudden changes in frequency. Circ Res 1969; 24:251-262.

2. Han J, Moe GK. Cumulative effects of cycle length on refractory periods in cardiac tissues. Am J Physiol 1969; 217:106-109

3. Wellens HJJ. Value and limitations of programmed electrical stimulation of the heart in the study and treatment of tachycardias. Circulation 1978; 57:845-853.

4. Franz MR, Swerdlow CD, Liem LB, et al. Cycle length dependence of human action-potential duration in-vivo. J Clin Invest 1988; 82:972-979.

5. Rickards AF, Norman J. Relation between QT interval and heart rate. New design of physiologically adaptive cardiac pacemaker. Br Heart J 1981; 45:56-61.

6. Morady F, Kadish AH, Toivonen LK, et al. The maximum effect of an increase in rate on human ventricular refracioriness. PACE 1988; 11:2223-2234.

7. Wiener I, Kunkes $S$, Rubin D, et al. Eflects of sudden change in cycle length on human atrial, atrioventricular nodal and ventricular refractory periods. Circulation 1981; 64:245-248.

8. Brownstein SI., Blackwell WH, Welch WJ, et al. Cumulative effects of cycle length on ventricular refractoriness in man. Am Heart J 1990; 119:324-330.

9. Boyett MR, Jewell BR. A study of the factors responsible for rate-dependent shortening of the action potential in mammalian ventricular muscle. J Physiol 1978; 285:359-380.

10. Franz MR, Platia EV, Schaefer J, el al. Fast and slow adaptation of human monophasic action potentials to abrupt changes in heart rate. (abstract) Am J Cardiol 1982; 49:970.

11. Franz MR, Long-term recording of monophasic action potentials from human endocardium. Am J Cardiol 1983; 51:1629-1634

12. Kanaan $\mathrm{N}$, Jenkins J, Childs $\mathrm{K}$, et al. Monophasic action-potential duration during programmed electrical stimulation. PACE 1991; 14:1049-1059.

13. Rosenbaum DS, Kaplan DT, Kanai A, el al. Repolarization inhomogeneities in ventricular myocardium change dynamically with abrupt cycle-lengh shortening. Circulation 1991; 84:1333-1345.

14. Lau CP, Freedman AR, Fleming 5 , et al. Hysteresis of the ventricular paced QT interval in response to abrupt changes in pacing rate. Cardiovasc Res 1988; 22:67-72.

15. Millar CK, Kralios FA, Lux RL. Correlation between refractory periods and activation-recovery intervals from electrograms: effects of rate and adrenergic interventions. Circulation 1985; 72:1372-1379. 
16. Haws CW, Lux RL. Correlation between in-vivo transmembrane action-potential durations and activation-recovery intervals from electrograms. Circulation 1990; 81:281-288.

17. Manley BS, Chong EMF, Walton C, et al. An animal model for the chronic study of ventricular repolarisation and refractory period. Cardiovasc Res 1989;23:16-20.

18. Morgan JM, Cunningham AD, Rowland E. Relationship of the effective relractory period and the monophasic action-potential duration alter a step increase in pacing frequency. PACE 1990; 13:1002-1008

19. Taggert $P$, Sutton $P$, Donaldson R. d-Solalol: a new potent class III anti-arhyythmic agent. Clinical Science 1985; 69:631-636.

20. Schmitt C, Brachmann J, Karch M, et al. Reverse use-dependent effects of sotalol demonstrated by recording monophasic action potentials of the right ventricle. Am J Cardiol 1991; 68:118311.87.

21. Huikuri HV, Yli-Mäyry S. Frequency dependent effects of d-sotalol and amiodarone on the action-potential duration of the human right ventricle. PACE 1992; 15(Pt. II):2103-2107.

22. Ruffy R. Sotalol. J Cardiovasc Electrophysiol 1993; 4:81-98.

23. Scherlag BJ, Kosowsky BD, Damato AN. A technique for ventricular pacing from the His bundle in the intact heart. J Appl Physiol 1967; 22:584-587.

24. Morady F, Kadish AH, Rosenheck S, et al. Elfect of the intertrain pause on the ventricular eflective refractory period measured by the extrastimulus technique. PACE 1990; 13:405-409.

25. Leerssen $\mathrm{HM}$, Vos MA, den Dulk $\mathrm{K}$, et al. Is the ventricular effective tefractory period different when determined by incremental versus decremental scanning?: the effect of pacing cycle length, d-sotalol and levcromakalim. PACE 1994; 17(Pt. II):2084-2089.

26. Edvardsson N, Hirsch I, Olsson $\$ B$. Right ventricular monophasic action potentials in healthy young men. PACE 1984: 7(Pt. V):813-821.

27. Baig $M W$, Boute $W$, Begemann $M$, et al. Nonlinear relationship between pacing and evoked $Q T$ intervals. PACE $1988 ; 11: 753-759$

28. Leerssen $\mathrm{HM}$, Vos $\mathrm{MA}$, den Dulk $\mathrm{K}$. et al. Inter- and intraindividual variations in shorlening of ventricular effective refractory period after an abrupt decrease in pacing cycle length. PACE 1994; 17(Pt. II):2079-2083.

29. Elimow IR, Huang DT, Rend JM, et al. Optical mapping of repolarization and refractoriness from intact hearts. Circulation 1994;90:1469-1480.

30. Kanai A, Salama G. Optical mapping reveals that repolarization spreads anisotropically and is guided by fiber orientation in guinea pig hearts. Circ Res 1995; 77:784-802.

31. Marchlinski FE. Characterization of oscillations in ventricular relractoriness in man after an abrupt increment in heart rate. Circulation 1987; 75:550-556.

32. Verduyn SC, Vos MA, van der Zande J, el al. Biventricular hypertrophy ficilitales occurrence of acquired torsade de pointes arthythnias in dogs. (abstract) Circulation 1995; 92:504-505.

33. Denardo S], Stevenson WG. Is the adaptation of right ventricular refractoriness to an abrupt increase in heart rate impaired in chronic heart lailure? Am Heart J 1989; 117:585-589.

34. Donaldson RM, Rickards AF Evaluation of drug-induced changes in myocardial repolarisation using the paced evoked response. Br Heart J 1982; 48:381-387.

35. Donaldson RM, Taggert P, Swanton $H$, el al. Intracardiac clectrode delection of early ischaemia in man. Br Heart J 1983; 50:213-221. 
36. Belz MK, Ellenbogen KA, Camm AJ, et al. Differentiation between monomorphic ventricular tachycardia and sinus tachycardia based on the right ventricular evoked potential. PACE 1992; 15(Pt. 1):1661-1666.

37. Bolz A, Hardt R, Hubmann M, et al. A new autocapture logic based on fractally coated low polarization pacing leads. (abstract) PACE 1993; 16:1914. 


\section{Chapter 6}

Rate-Dependent Effects of Procainamide on the Threshold Current for Pacing in the Setting of Postrepolarization Refractoriness in Dogs

Hendrik M. Leerssen, Marc A. Vos, Karel den Dulk, Jolanda van der Zande, and Hein J.J. Wellens.

Department of Cardiology, Cardiovascular Research Institute Maastricht, Maastricht University, The Netherlands.

Published in PACE 1999; 22:291-301. 


\begin{abstract}
Introduction: Normally, ventricular action-potential duration (APD) exceeds the ventricular effective refractory period (VERP). However, under specific circumstances this relation may change and can become inverse. This phenomenon of postrepolarization refractoriness may be caused by a decrease in excitability. The threshold current (IC) for pacing has never been quantified as a possible explanation for these observations.

Methods and Results: Using a monophasic action-potential (MAP) pacing catheter in the right ventricular apex, the rate-dependent behavior of TC, VERP, and APD before and after procainamide (dose $20 \mathrm{mg} / \mathrm{kg}$ in $10 \mathrm{~min}+5 \mathrm{mg} / \mathrm{min}$ infusion) was determined in 17 dogs with chronic complete atrioventricular block. Initially, TC [78] was determined with $0.1 \mathrm{~mA}$ accuracy. Using a pacing current of at least twice TC, VERP and APD showed a similar, rate-dependent shortening for pacing cycle lengths (PCLs) 800,575 , and $350 \mathrm{msec}$. Procainamide treatment led to an equal, rate independent VERP and APD increase: no postrepolarization refractoriness. Subsequently, accuracy for TC determination was increased to $0.01 \mathrm{~mA}$. Comparing PCLs 800 and $250 \mathrm{msec}$, TC doubled from $0.05 \pm 0.01$ to $0.10 \pm 0.09$ $\mathrm{mA}$ during control and almost tripled from $0.06 \pm 0.02$ to $0.17 \pm 0.10 \mathrm{~mA}(\mathrm{P}<$ 0.05 ) after procainamide. Using a fixed pacing current of exactly twice IC found at $800 \mathrm{msec}$ PCL during control, VERP exceeded APD after procainamide treatment at 300 and 250 msec PCL: postrepolarization refractoriness. Increasing the pacing current to twice the rate-dependent TC, the relation between VERP and APD normalized: no postrepolarization refractoriness.

Conclusion: After procainamide, rate-dependent TC increase is of major importance for the phenomenon of postrepolarization refractoriness.
\end{abstract}




\section{Introduction}

Monophasic action-potential (MAP) recordings reproduce the repolarization time course of intracellular action potentials of the myocardial cells with a high accuracy. Compared to the "gold standard" of the transmembrane action potential in isolated cells or tissue, the main advantage of MAPs is its in-vivo applicability, bridging the gap between basic and clinic electrophysiology.1-4

In atrial and ventricular myocardium, in the absence of ischemia and antiarrhythmic drugs, repolarization normally outlasts refractoriness. In other words: the action-potential duration (APD) is longer than the ventricular effective refractory period (VERP), leading to a ratio of VERP and APD (VERP/APD) of around $0.9 .5,6$

Using a quadripolar contact electrode that enabled simultaneous determinations of VERP and APD at closely adjacent endocardial sites, the difference between VERP and APD was shown to be constant over a wide range of pacing cycle lengths (PCLs) in dogs and humans. ${ }^{7}$ Changes in the difference between VERP and APD, e.g. due to lengthening of VERP which is not accounted for by identical lengthening of APD, can lead to what has been called postrepolarization refractoriness. Postrepolarization refractoriness has been described under ischemic conditions in vitro ${ }^{8,9}$ and at critical PCLs, after administration of sodium channel blocking drugs such as procainamide or quinidine. Under these circumstances, VERP could even become longer than APD, leading to VERP/APD > 1 .

This finding of postrepolarization refractoriness was explained by a rate or use dependent decrease in ventricular excitability, ${ }^{5,6,10-13}$ The decrease in excitability will lead to an increase in threshold current (TC) and if the pacing current is not adapted accordingly, also to lengthening of VERP.

Some authors 11,13 indicated that the increase in VERP could be used as an indirect measure for the decrease in excitability. However, at a certain heart rate, VERP duration is not only determined by the excitability of the tissue, but also by the pacing protocol, the current strength, the atrioventricular relationship, and the polarity of pacing. 14-21 Therefore an increase in VERP suggests but does not quantify a decrease in excitability.

The aim of this study was to quantify the rate-dependent behavior of TC, VERP, and APD before and after administration of the Class IA antiarthythmic drug procainamide at PCL $800,575,350,300$, and $250 \mathrm{msec}$. This was done in 17 dogs with induced chronic complete atrioventricular (AV) block. Furthermore the relevance of the relation between TC increase and pacing current for the development of postrepolarization refractoriness was studied. 


\section{Methods}

This protocol was approved by the local ethical committee for experiments on animals, and conducted in accordance with the guidelines for experiments on animals of the American Physiological Society.

\section{Definitions}

Threshold current (TC): The lowest current during diastole allowing consistent $(>30$ s) ventricular capture.

Ventricular effective refractory period (VERP): The longest coupling interval of a premature test pulse not capturing the heart. The pacing protocol applied will be described in the Methods section "electrical recordings, pacing and protocols."

[80] Action-potential duration (APD): The interval from the beginning of the upstroke of the local activation to the end of repolarization (APD100) as measured in the MAP recording.

Postrepolarization refractoriness: A change in VERP that is not accounted for by an identical change in APD leading to an increase in VERP/APD.

\section{Preparation}

Seventeen experiments were performed in 17 anesthetized adult mongrel dogs of either sex, with induced chronic, complete AV block. These 17 dogs were divided into three groups of which the characteristics are shown in Table 6.1.

At least 2 weeks before the actual experiment (Table 6.1), permanent complete AV block was induced by injecting $37 \%$ formaldehyde into the AV junction without atriotomy after a right thoracotomy. ${ }^{22}$ During the actual experiment a MAP-pacing catheter (Franz MAP-Pacing catheter, EP Technologies Inc., Mountain View, CA, USA) was introduced via the jugular or femoral vein and positioned with the help of fluoroscopy in the apical region of the right ventricle.

AV block induction as well as the actual experiment were performed under sterile conditions and antibiotic cover. After intramuscular premedication, anesthesia was induced by intravenous sodium pentobarbitone $(20 \mathrm{mg} / \mathrm{kg}$ ). The dogs were artificially ventilated through a cuffed endotracheal tube with a mixture of oxygen, nitrous oxide, and halothane (vapor concentration $0.5-1 \%$ ). Ventilation was adjusted on the basis of continuous monitoring of the carbon dioxide concentration of the expired air. A thermal mattress was used to maintain body temperature at approximately $37^{\circ}$ Celsius.

Procainamide was administered as a bolus of $20 \mathrm{mg} / \mathrm{kg}$ in $10 \mathrm{~min}$ followed by a $5 \mathrm{mg} / \mathrm{min}$ infusion using the cephalic vein. This infusion regimen was designed to attain steady-state plasma concentrations in dogs of $10-15 \mu \mathrm{g} / \mathrm{ml}$ resulting in an increase in VERP of approximately 10\%.23.24

\section{Threshold Current and Pacing Current for VERP Determination}

Group I: TC was determined during control at $800 \mathrm{msec} P C L$ with $0.1 \mathrm{~mA}$ precision. VERP was determined with a fixed current of $0.5 \mathrm{~mA}$ irrespective of the 
effect of PCL change and procainamide (Table 6.1).

Group II: To test the effect of procainamide treatment on TC, TC was determined before and after procainamide treatment at $800 \mathrm{msec} P C L$ with $0.1 \mathrm{~mA}$ precision. VERP was determined with a current of twice the TC found at $800 \mathrm{msec}$ irrespective of PCL, before and after procainamide (Table 6.1).

Group III: To test the effect of PCL and procainamide treatment on TC, TC was determined during control and after procainamide treatment at all PCLs with $0.01 \mathrm{~mA}$ precision. During control, VERP was determined with a current of twice the IC found at $800 \mathrm{msec}$, irrespective of PCL. After procainamide treatment VERP was determined with a current of twice the TC found at $800 \mathrm{msec}$ during control and at twice the rate-dependent TC (Table 6.1).

Specifications of the MAP Pacing Catheter and Stimulator

TC, VERP, and APD were determined simultaneously at the same right ventricular endocardial site with the MAP pacing catheter. The two sintered $\mathrm{Ag}-\mathrm{AgCl}$ electrodes of this quadripolar catheter are used for MAP recording, one is positioned on the tip and the other, a ring, $4 \mathrm{~mm}$ proximal to the tip. For pacing two small platinum electrodes with $2 \mathrm{~mm}$ electrode spacing are positioned opposite to each other close to the tip between the MAP recording electrodes.

Table 6.1 Characteristics of dogs and pacing

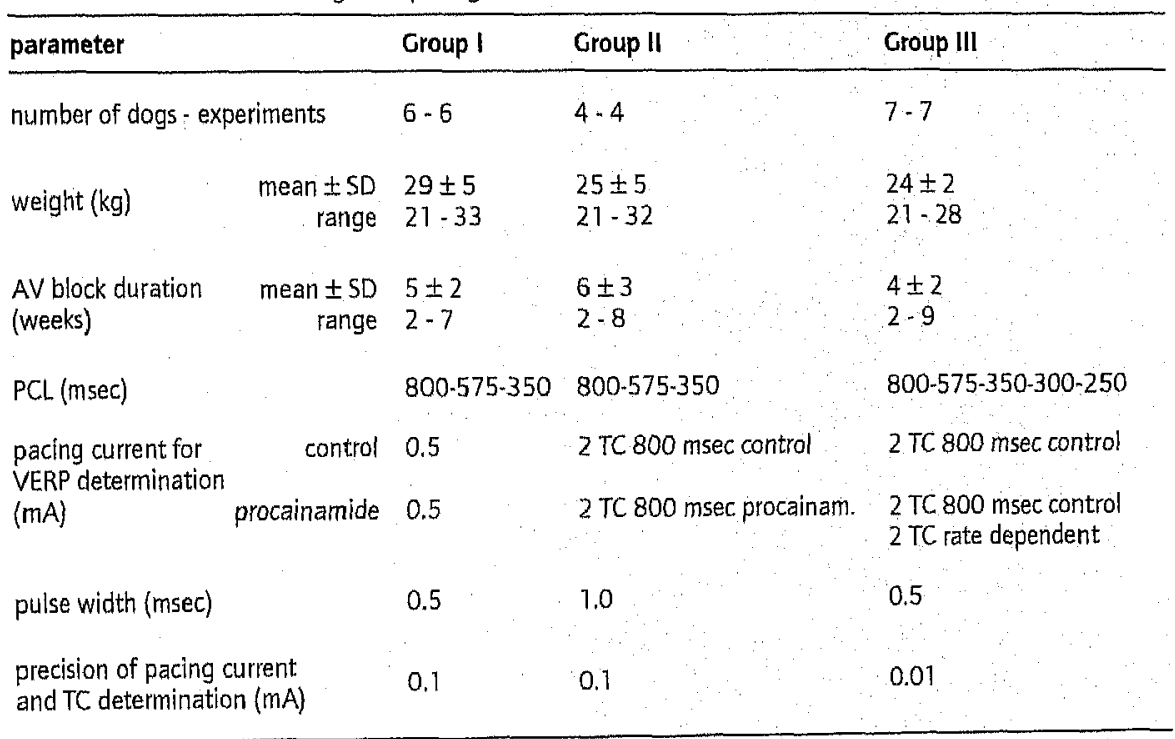

Abbreviations: $A V=$ atrioventricular, $P C L=$ pacing cycle length; $T C=$ threshold current; $V E R P=$ ventricular effective refractory period. 2 TC $800 \mathrm{msec}$ control = twice the threshold current determined at $800 \mathrm{msec}$ pacing cycle length during control conditions; 2 TC $800 \mathrm{msec}$ procainam. = twice the threshold current determined at $800 \mathrm{msec}$ pacing cycle length after procainamide treatment; 2 TC rate dependent $=$ twice the threshold current determined at the actual pacing cycle length and conditions. 
The equipment to determine current duration and intensity was custom built developed in collaboration with the Department of Medical Technology, Maastricht University, Maastricht, The Netherlands) Timing and duration of pacing was provided by a trigger source based on a $8 \mathrm{MHz}$ crystal. This trigger source guarantees the value of the programmed pulse duration $\pm 1 \mu \mathrm{s}$. A medically safe precision power supply class CF (IEC 601) was used to set the desired current intensity. Nevertheless, the actual current delivered was measured on a beat-tobeat basis with $0.01 \mathrm{~mA}$ precision. Deviations between the delivered and desired current intensity were corrected immediately.

The MAP was amplified by a direct current coupled amplifier, which has a $20 \mathrm{mV}$ calibration pulse. The minimum amplitude for acceptance of the signal was $15 \mathrm{mV}^{3}$

Pacing Protocol for VERP Determination

The protocol used to determine VERP has been described before as nointertrain pause incremental scanning. ${ }^{14,16,21}$ Briefly, a test pulse was introduced after every eighth beat in the continuous pacing train. Initially the coupling interval of this test pulse was shorter than VERP. Therefore the test pulse did not capture and steady-state circumstances were not disturbed. Subsequently the coupling interval of the test pulse was incremented by $5 \mathrm{msec}$ until capture occurred. Thereafter the coupling interval of the test pulse was reduced to a value shorter than VERP and incremented until capture reoccurred. This procedure was repeated until two identical values for VERP were found. The current for the continuous pacing train and the test pulse was identical. Measurements at a certain PCL did not start before a conditioning period of at least 3 minutes.

\section{Data Acquisition and Analysis}

All signals were sampled at a rate of $1 \mathrm{kHz}$ and stored on hard disk with six surface ECG leads. APD was determined via off-line analysis using "ECG-view" software (developed in collaboration with the Department of Medical Technology, Maastricht University, Maastricht, The Netherlands). The settings of this software allowed APD determination with $5 \mathrm{msec}$ accuracy. APD was determined as the mean of 5 consecutive APDs during regular pacing after steady-state circumstances had been reached. VERP was determined with $5 \mathrm{msec}$ accuracy.

\section{Statistics}

The software used for the statistical tests was Primer of Biostatistics (McGraw-Hill Inc., New York, NY, USA) The outcome of the tests was considered statistically significant for $\mathrm{P}<0.05$.

In groups I, II, and III (Tables 6.2 and 6.3) a paired t-test was used to test statistical significance of procainamide treatment on VERP, APD, and VERP/APD.

A Wilcoxon signed rank test was used to test the statistical significance of: (1) the rate-dependent $T C$ increase during control and after procainamide treat- 
ment compared to the 800 msec PCL TC (Figure 6.1); and (2) the TC increase after procainamide treatment compared to the matching PCLs during control (Figure 6.1).

\section{Results}

\section{Group I}

During control at $800 \mathrm{msec}$ PCL, TC (determined with $0.1 \mathrm{~mA}$ precision) was $0.1 \mathrm{~mA}$ in 4 dogs and $0.2 \mathrm{~mA}$ in 2 dogs. Using a fixed pacing current of 0.5 $\mathrm{mA}$, a rate-dependent shortening of VERP and APD before and after procainamide treatment was found (Table 6.2). Procainamide treatment led to a significant increase in VERP and APD at the PCLs tested $(800,575$, and $350 \mathrm{msec})$ but VERP/APD remained unchanged and smaller than 1 : no postrepolarization refractoriness (Table 6.2).

\section{Group II}

During control at $800 \mathrm{msec}$ PCL, TC (determined with $0.1 \mathrm{~mA}$ precision) was $0.1 \mathrm{~mA}$ in all clogs. After procainamide treatment, IC at 800 msec PCL increased respectively to 0.2 and $0.4 \mathrm{~mA}$ in two dogs; the rest remained at $0.1 \mathrm{~mA}$. Observations in group II were very similar to group I (Table 6.2). As in group I,

Table 6.2 The effect of procainamide and PCI. on VERP, APD, and VERP/APD.

\begin{tabular}{|c|c|c|c|c|c|}
\hline \multirow[b]{2}{*}{ PCL (msec) } & & \multicolumn{2}{|l|}{ group 1} & \multicolumn{2}{|l|}{ group II } \\
\hline & & control & procainamide & control & procainamide \\
\hline \multirow[t]{3}{*}{800} & VERP (msec) & $235 \pm 28$ & $263 \pm 30$ * & $231 \pm 11$ & $280 \pm 76 *$ \\
\hline & $\mathrm{APD} \quad$ (msec) & $273 \pm 38$ & $305 \pm 35$ * & $259 \pm 19$ & $310 \pm 35$ * \\
\hline & VERP/APD & $0.86 \pm 0.05$ & $0.86 \pm 0.04$ & $0.90 \pm 0.10$ & $0.91 \pm 0.14$ \\
\hline \multirow[t]{3}{*}{575} & VERP (msec) & $208 \pm 20$ & $228 \pm 20 *$ & $209 \pm 9$ & $245 \pm 16$ * \\
\hline & APD (msec) & $245 \pm 26$ & $268 \pm 28 *$ & $230 \pm 20$ & $271 \pm 33 *$ \\
\hline & VERP/APD & $0.85 \pm 0.02$ & $0.85 \pm 0.04$ & $0.91 \pm 0.10$ & $0.92 \pm 0.16$ \\
\hline \multirow[t]{3}{*}{350} & VERP (msec) & $167 \pm 14$ & $187 \pm 14$ * & $169 \pm 10$ & $204 \pm 18 *$ \\
\hline & APD (msec) & $209 \pm 21$ & $225 \pm 19 *$ & $194 \pm 20$ & $219 \pm 35 *$ \\
\hline & VERP/APD & $0.80 \pm 0.07$ & $0.83 \pm 0.06$ & $0.88 \pm 0.13$ & $0.95 \pm 0.26$ \\
\hline
\end{tabular}

Abbreviations: $P C L=$ pacing cycle length; $V E R P=$ ventricular effective refractory period; $A P D=$ actionpotential duration. Values as mean \pm SD. A significant difference of VERP, APD, and VERP/APD during procainamide compared to control is indicated by * (paired $t$ test, $\mathrm{P}<0.05$ ). 
Table 6.3 The effect of pacing current, procainamide and PCL on VERP, APD, and VERP/APD in group III.

\begin{tabular}{|c|c|c|c|c|c|c|c|c|c|}
\hline & & pacing curre & & & & & & & \\
\hline & & $2 \mathrm{TC} .800 \mathrm{~ms}$ & ec cont & & & 2 TC rate de & pendent & & \\
\hline PCL & msec) & control & & procainamide & & control & & procainamid & \\
\hline 800 & VERP(msec) & $258 \pm 14$ & $n=7$ & $301 \pm 21 *$ & $n=7$ & $258 \pm 14$ & $n=7$ & $288 \pm 16$ * & $n=7$ \\
\hline & APD (msec) & $295 \pm 27$ & 7 & $319 \pm 24 *$ & 7 & $295 \pm 27$ & 7 & $319 \pm 24$ * & 7 \\
\hline & VERP/APD & $0.88 \pm 0.05$ & & $0.95 \pm 0.08$ & & $0.88 \pm 0.05$ & & $0.91 \pm 0.04$ & \\
\hline 575 & $\operatorname{VERP}$ (msec) & $231 \pm 10$ & 7 & $266 \pm 18 *$ & 7 & $231 \pm 9$ & 7 & $259 \pm 11^{*}$ & $?$ \\
\hline & APD (msec) & $266 \pm 21$ & 7 & $286 \pm 21 *$ & 7 & $266 \pm 21$ & 7 & $286 \pm 21$ * & 7 \\
\hline & VERP/APD & $0.87 \pm 0.04$ & & $0.93 \pm 0.06$ & & $0.87 \pm 0.04$ & & $0.91 \pm 0.05^{*}$ & \\
\hline 350 & $\operatorname{VERP}(\mathrm{msec})$ & $191 \pm 15$ & 7 & $231 \pm 22 *$ & 6 & $191 \pm 12$ & 7 & $217 \pm 10^{*}$ & 7 \\
\hline & APD (msec) & $221 \pm 16$ & 7 & $234 \pm 18$ * & 7 & $221 \pm 16$ & 7 & $234 \pm 18$ * & 7 \\
\hline & VERP/APD & $0.87 \pm 0.08$ & & $1.00 \pm 0.16$ & & $0.87 \pm 0.07$ & & $0.93 \pm 0.07 *$ & \\
\hline 300 & VERP(msec) & $176 \pm 13$ & 6 & $231 \pm 24 *$ & 6 & $182 \pm 20$ & 7 & $213 \pm 20^{*}$ & 7 \\
\hline & APD (msec) & $205 \pm 14$ & 7 & $219 \pm 16$ * & 7 & $205 \pm 14$ & 7 & $219 \pm 16 *$ & 7 \\
\hline & VERP/APD & $0.87 \pm 0.08$ & & $1.07 \pm 0.16$ * & & $0.89 \pm 0.09$ & & $0.97 \pm 0.12$ & \\
\hline 250 & VERP(msec) & $163 \pm 13$ & 4 & $205 \pm 7 *$ & 2 & $155 \pm 13$ & 6 & $179 \pm 11^{*}$ & 4 \\
\hline & APD (msec) & $185 \pm 12$ & 6 & $197 \pm 20$ * & 5 & $185 \pm 12$ & 6 & $197 \pm 20$ * & 5 \\
\hline & VERP/APD & $0.91 \pm 0.10$ & & $1.05 \pm 0.04$ & & $0.84 \pm 0.17$ & & $0.95 \pm 0.09$ & \\
\hline
\end{tabular}

Abbreviations: $T C=$ threshold current; $P C L=$ pacing cycle length; $V E R P=$ ventricular effective refractory period; $\mathrm{APD}=$ action-potential duration; $n=$ the amount of dogs; $2 \mathrm{TC} 800 \mathrm{msec}$ control $=$ twice the threshold current determined at 800 msec pacing cycle length during control conditions; $2 \mathrm{TC}$ rate dependent = twice the threshold current determined at the actual pacing cycle length and conditions. Values as mean \pm SD. A significant difference for VERP, APD, and VERP/APD after procainamide compared to control is indicated by * (paired $t$ test, $\mathrm{P}<0.05$ ).

procainamide treatment led to a significant increase in VERP and APD at the PCLs tested $(800,575$, and $350 \mathrm{msec})$, without changing the relation between VERP and APD (VERP/APD < 1): no postrepolarization refractoriness (Table 6.2).

\section{Group III}

The TC of the MAP-pacing catheter was very low at all PCLs tested, not only during control but also after procainamide treatment (Figure 6.1). Under control conditions, TC determined with $0.01 \mathrm{~mA}$ precision demonstrated a ratedependent doubling from $0.05 \pm 0.01 \mathrm{~mA}$ at $800 \mathrm{msec}$ PCL to $0.10 \pm 0.09 \mathrm{~mA}$ at $250 \mathrm{msec}$ PCL (Figure 6.1). However, this increase did not reach statistical significance. After procainamide treatment, TC increased at the short PCLS, demon- 


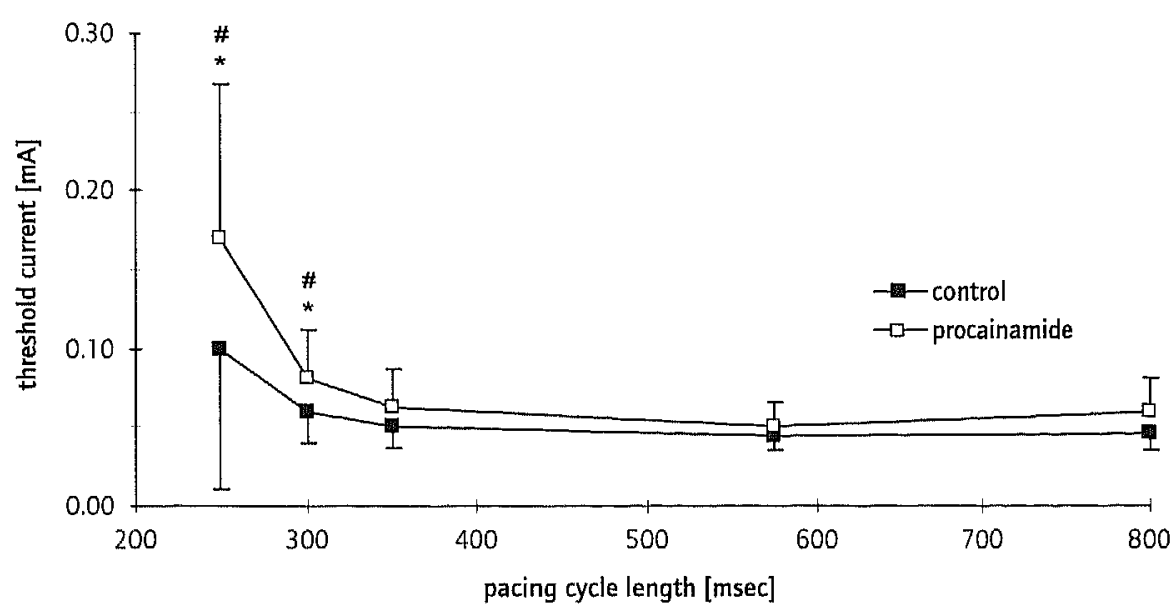

Figure 6.1 - The effect of PCL on the TC of the MAP pacing catheter in group III.

A significant difference compared to $800 \mathrm{msec} P C L$ is indicated by * (Wilcoxon signed rank test, $P<0.05$ ). A significant difference compared to matching PCLs during control is indicated by \# (Wilcoxon signed rank test, $P<0.05$ ). Abbreviations: $P C L=$ pacing cycle length; $T C=$ threshold current; $M A P=$ monophasic action potential.

strating a significant rate dependency; TC almost tripled from $0.06 \pm 0.02 \mathrm{~mA}$ at $800 \mathrm{msec}$ PCL to $0.17 \pm 0.10 \mathrm{~mA}$ at $250 \mathrm{msec}$ PCL (Figure 6.1).

As in groups 1 and II, a rate-dependent shortening of APD and VERP was found before and after procainamide treatment (Table 6.3). After procainamide treatment VERP and APD were determined with two settings of the pacing current: twice TC $800 \mathrm{msec}$ control (Table 6.3, left panel) and twice rate-dependent TC (Table 6.3, right panel). Although APDs for the two settings of the pacing current were independently acquired, the values were identical for the matching PCLs during control and after procainamide.

Using twice rate-dependent TC, VERP/APD after procainamide treatment showed a significant increase at 575 and 350 msec PCL compared to control. Furthermore the observations were very similar to groups I and II for the PCLS that could be compared $(800,575$, and $350 \mathrm{msec})$ : APD was always longer than VERP and therefore VERP/APD $<1$. The reduction in number of experiments (n in Table 6.3) at $250 \mathrm{msec}$ PCL was related to the limited time dogs could be paced at this PCL.

Using twice TC $800 \mathrm{msec}$ control, VERP/APD after procainamide treatment showed a significant increase at $300 \mathrm{msec}$ PCL compared to control. More importantly, stimulation with twice TC $800 \mathrm{msec}$ control induced two major differences at the shortest PCLs compared to stimulation with twice rate-dependent TC: (1) VERP increased more after procainamide treatment and became longer than APD at 300 and $250 \mathrm{msec}$ PCL and therefore VERP/APD > 1: postrepolarization refractoriness (Table 6.3); and (2) in many dogs, pacing did not capture because the 
rate-dependent $T C$ at the short $P C L$ was higher than the pacing current of twice TC $800 \mathrm{msec}$ control. This is illustrated by the increased reduction in number of experiments compared to twice rate-dependent TC ( $\mathrm{n}$ in Table 6.3). This TC behavior, leading to postrepolarization refractoriness, is illustrated in Figure 6.1.

\section{Discussion}

While APD is mainly determined by local tissue properties, as illustrated by identical values for different pacing currents (Table 6.3), VERP is also determined by many external factors. An increase in the pacing current can lead to a shorter value of VERP; the length of the conditioning pacing train which precedes the pre-

[86] mature pacing pulse for VERP determination together with the pauses between subsequent pacing trains can influence VERP; incremental compared to decremental scanning of the premature stimuli can have an effect on VERP and, furthermore, the atrioventricular relationship and the polarity of pacing are known to play a role. ${ }^{1+-21}$

Normally, VERP is determined with twice TC. This is done to assure constant capture and to increase reproducibility. Often this TC is determined at a relatively long PCL during control conditions. For normal pacing leads TC can be as low as $0.25 \mathrm{~mA}$ in the acute phase. ${ }^{25}$ Therefore, the margin of doubling the TC, would be at least $0.25 \mathrm{~mA}$. The MAP pacing catheter, can have a TC as low as 0.05 $\mathrm{mA}$. This is related to the small surface area $\left(2 \mathrm{~mm}^{2}\right)$ of the pacing bipole and its position $2 \mathrm{~mm}$ proximal to the tip that probably leads to close contact with the endocardium. 7.25 Values of $0.099 \pm 0.051 \mathrm{~mA}$ (range $0.02-0.25 \mathrm{~mA}, \mathrm{n}=36)^{7}$ have been reported in dogs. In patients, TCs in the right ventricular apex respectively right ventricular outflow tract of $0.18 \pm 0.20 \mathrm{~mA}(\mathrm{n}=5)^{7}$ and $0.12 \pm 0.05 \mathrm{~mA}$ $(n=21)^{26}$ have been reported. A TC of $0.05 \mathrm{~mA}$ will lead to a margin of only 0.05 $\mathrm{mA}$. Using the MAP-pacing catheter in a situation in which IC increases, as can be the case after Class IA antiarrhythmic medication at short PCLs, a non corrected pacing current can lead to longer values for VERP.

\section{The Effect of PCL on VERP, APD, and TC}

Under normal circumstances, VERP is shorter than APD. In the literature a difference between VERP and $\mathrm{APD}_{90}$ has been reported around - $10 \mathrm{msec}$ in isolated papillary muscle of guinea pigs, ${ }^{12}$ in dogs, ${ }^{11.27}$ and in humans ${ }^{13}$ leading to a VERP/APD of approximately 0.9.5, Both the difference between and the ratio of VERP and APD are constant for a wide range of PCLs. $5-7,11,13,27$

The results of the experiments in groups I, II, and III (Tables 6.2 and 6.3) are in accordance with these findings. During control conditions we found a VERP/APD between $0.80-0.91$ for PCLs as short as $250 \mathrm{msec}$. Since we used $A P D_{100}$ this longer APD might be the reason for the lower values compared to the literature. 
In the literature the amount of data with respect to rate-dependent changes in TC in the absence of antiarrhythmic medication is limited and sometimes conflicting; some authors report a rate-dependent increase in TC while others do not. 13,28 We quantified the rate-dependent TC using a MAP pacing catheter with $0.01 \mathrm{~mA}$ accuracy. Although the increase did not reach statistical significance, TC doubled from 0.05 to $0.10 \mathrm{~mA}$ when comparing PCLs 800 and $250 \mathrm{msec}$, indicating a rate dependency of TC (Figure 6.1).

The Effect of Procainamide on VERP, APD, and TC

In groups I, II, and III procainamide treatment leads to a significant increase in VERP and APD (Tables 6.2 and 6.3) at all the PCLs tested. In the literature several reports can be found in this respect in humans; mean values for VERP lengthening, determined in the right ventricular apex, mostly at a PCL of approximately $600 \mathrm{msec}$, range from 17 to $42 \mathrm{msec}$, which is in accordance with our data. 10,29,30

The effect of procainamide, or other Class IA antiarrhythmic drugs such as quinidine, on APD, has often been studied in combination with VERP.5,6,11 These studies showed an increase in APD very similar to the increase in VERP. Lee et al,13 observed no rate-dependent behavior of the difference between VERP and APD during control at all PCLs tested and after procainamide treatment at PCLs ranging from 600 to $450 \mathrm{msec}$. At PCLs 400 and $350 \mathrm{msec}$, VERP increased more than $A P D$ after procainamide treatment, giving rise to postrepolarization refractoriness. We could confirm this rate-dependent increase in VERP after procainamide treatment in group III, using a pacing current which was fixed to the value determined at $800 \mathrm{msec}$ PCL during control.

We studied the literature with respect to the quantification of changes in TC (of a MAP pacing catheter) after treatment with Class IA antiarrhythmic drugs. No significant TC changes were found at therapeutic levels of Class IA antiarrhythmic drugs. ${ }^{10,31}$ However, procainamide toxicity might cause an excessive rise in TC with failure to capture. $32-34$ Other authors found a TC increase which was rate-dependent in humans ${ }^{13}$ and dogs, ${ }^{11}$ however the rate-dependent TC increase was never quantified. Therefore, we quantified the rate-dependent TC after procainamide treatment. For the longer PCLs $(800,575$, and $350 \mathrm{msec})$ no increase was found whereas for the shorter PCLs (300 and $250 \mathrm{msec}$ ) TC increased significantly (Figure 6.1). Although TC for the shortest PCL almost tripled compared to the longer PCLs the absolute increase in TC is limited and could be less than the resolution of the measurement. TC determination with insufficient accuracy might be the reason the increase in TC is not always found. ${ }^{35}$

\section{The Interaction Between Pacing Current and Postrepolarization Refractoriness}

Under specific circumstances, the relation between VERP and APD may change: VERP can become equal to or longer than APD. ${ }^{11}$ This situation of postrepolarization refractoriness was demonstrated at short cycle lengths under 
ischemic conditions in canine His bundle ${ }^{8}$ and Purkinje fibers ${ }^{9}$ and after administration of Class IA antiarrhythmic drugs at short PCLs, both in humans ${ }^{6,13}$ and canines. " As an expression of postrepolarization refractoriness the difference between VERP and APD changed from negative to less negative or even positive values ${ }^{11-13.27}$ and VERP/APD increased to values above $1.5,6$ The increase in the rate-dependent $T C$ in the setting of postrepolarization refractoriness was never quantified.

From the literature it is known that the development of postrepolarization refractoriness is strongly related to a decreased myocardial excitability which can develop during ischemia and after Class IA antiarrhythmic drugs at short PCLs. $5,6,9-13,32,33$ Therefore, a pacing current that compensates for this decrease in excitability should restore the normal relation between VERP and APD.

In group III, using a pacing current which was fixed to the value determined at $800 \mathrm{msec}$ PCL during control, VERP lengthening after procainamide treatment was rate-dependent. At the shortest PCLs (300 and $250 \mathrm{msec}$ ) VERP increased significantly more and even exceeded APD compared to the longer PCLs (Table 6.3, left panel). As a result the difference between VERP and APD changed from negative to positive values (VERP/APD $>1$ ): postrepolarization refractoriness. This effect is blunted at $250 \mathrm{msec}$ PCL. At $250 \mathrm{msec}$ PCL alter procainamide treatment, TC often increased to a level which exceeded twice the TC determined at $800 \mathrm{msec}$ PCL during control. Because this leads to loss of capture, VERP measurements can not be performed.

Using a pacing current that was constantly adapted to the rate-dependent TC, VERP lengthening after procainamide treatment was not rate-dependent (Table 6.3, right panel) and the difference between VERP and APD did not show the change to positive values. The normal relation between VERP and APD was restored (VERP/APD < 1): no postrepolarization refractoriness. In groups I and II, using an overestimated pacing current the same results were found for the PCLs tested (Table 6.2).

\section{Prerequisites for Postrepolarization Refractoriness Detection}

Using the MAP pacing catheter we found TCs as low as $0.05 \mathrm{~mA}$ at a long $\mathrm{PCL}$ during control conditions (Figure 6.1). The margin of doubling the pacing current would only be $0.05 \mathrm{~mA}$, presumed that TC and pacing current could be determined with $0.01 \mathrm{~mA}$ accuracy.

At short PCLs, after treatment with antiarrhythmic drugs like procainamide, TC can easily increase with $0.10 \mathrm{~mA}$ compared to TC at the long PCL during control (Figure 6.1). If the pacing current for the normal pacing lead is not adapted, this will lead to a reduction of the safety margin. Since, in most of the cases, this reduction will be small compared to the margin of the pacing current, this will not lead to lengthening of VERP, thereby obscuring the development of postrepolarization refractoriness. This probably explains why some investigators did not find a rate-dependent increase in VERP after procainamide treatment. ${ }^{35}$ 
Using a MAP pacing catheter, with high precision pacing equipment, an increase in TC even smaller than $0.10 \mathrm{~mA}$ will already lead to VERP lengthening, thereby clearly demonstrating the development of postrepolarization refractoriness. An increase of $0.10 \mathrm{~mA}$ will even lead to loss of capture in most of the cases. This was confirmed in the experiments of group III. Using twice TC $800 \mathrm{msec}$ control (Table 6.3, left panel) the number of VERP measurements at 350, 300, and $250 \mathrm{msec}$ PCL was reduced because the rate-dependent TC exceeded the programmed pacing current.

\section{Cellular Determinants of Excitability and Refractoriness}

Normally in atrial, ventricular and His-Purkinje cells the time course of recovery of excitability closely follows that of membrane repolarization. Nevertheless, by the time the membrane voltage is fully repolarized, the availability of $\mathrm{Na}^{+}$channels is still not returned to baseline and not all $\mathrm{K}^{+}$channels are deactivated. As a result total recovery of excitability usually outlasts full membrane repolarization. ${ }^{36,37}$ This explains the increased TCs found in this study during control for the shortest PCLs: the coupling interval is shorter than the time required for total recovery of excitability.

After administration of procainamide membrane responsiveness will be decreased. ${ }^{29}$ Compared to control, the amount of postrepolarization refractoriness will increase.$^{37}$ In our study we confirmed that this will have no consequences for TC values determined during late diastole at long PCLs when the availability of fast $\mathrm{Na}^{+}$channels does not seem to be limiting. ${ }^{10,12}$ However, when PCL shortens, the interval between complete recovery of excitability and the next stimulus will shorten. ${ }^{5}$ At a certain PCL, this interval will no longer be present since the next stimulus is before complete recovery of excitability. ${ }^{12}$

\section{Limitations of the Study}

\section{Excitability and Refractoriness}

The rate-dependent TC in group I, II, and III was determined with a single fixed pulse width of $0.5-1.0 \mathrm{msec}$. This could be considered a shortcoming of this study since a strength duration curve determined at every PCL would gather a more complete understanding of the effect of rate and/or procainamide on excitability. However, such a study is difficult in-vivo with the necessity of continuous pacing at very short PCLs for longer periods which can result in hemodynamic deterioration in the hypertrophied heart of the AV block dog. 38 Furthermore, maintenance of a constant autonomic tone and plasma drug concentration throughout the measurements could become a problem.

Other investigators ${ }^{39}$ tested the effect of decreasing PCLs on human atrial strength duration curves. They found a shift upward and to the right with decreasing PCL. We expect the same behavior in the ventricle. The minimum current irrespective of the pulse duration, will increase (shift upward) and the minimum pulse width required for pacing, irrespective of the current will increase (shift to the right). 40 
Similar to the single pulse width for TC determination, VERP was determined with a single current strength because pacing at the shortest PCLs could not be continued for the required period to determine a complete strength duration curve. Nevertheless, it is clear that $\mathrm{PCL}$ decrease before and, especially, after procainamide treatment leads to a decrease in excitability. If the output is not adapted accordingly this can lead to an increase in VERP.40

Other investigators ${ }^{10}$ tested the effect of procainamide on the relation between current and VERP at a single PCL. They found that procainamide did not change the shape of the strength interval curve: increasing the stimulus strength resulted in a progressive VERP reduction until it approached an asymptotic value. Procainamide shifted the curve to the right; that is, it increased the refractory period at all current strengths and therefore decreased excitability. The basic strength-

[90] interval relation, however remains unaffected by procainamide.

Electrophysiologic Heterogeneity

The experiments were performed with a MAP pacing catheter at a right ventricular endocardial site. From the literature it is known that there is marked electrophysiologic heterogeneity not only between different endocardial sites in the ventricle ${ }^{41}$ and between the ventricles $5^{42}$ but also between endocardium and epicardium of the same ventricular site. ${ }^{43}$ Although this electrophysiologic heterogeneity will lead to different values for APD and VERP for different sites, we believe that there will be a very similar response of myocardial cells after PCL decrease and/or procainamide administration.

\section{Conclusion}

Under normal circumstances VERP is shorter than APD. Since the shortening of VERP and APD after a decrease in PCL is very similar, the difference between VERP and APD has a constant rate independent value. After procainamide treatment the normal relation between VERP and APD can be disturbed: after a critical PCL decrease VERP can show less shortening than APD. The difference between VERP and APD is reduced and VERP can even become longer than APD. As a result VERP/APD can increase to values above 1: postrepolarization refractoriness.

We quantified the rate-dependent TC of a MAP pacing catheter during control and during the circumstances that lead to postrepolarization refractoriness with high accuracy. After procainamide treatment, the TC showed a rate-dependent increase. When the pacing current was not adapted accordingly, but remained at the level determined at the long PCL during control, the shortening of VERP after a critical PCL decrease was reduced compared to the shortening of APD, VERP/APD increased to values above 1: postrepolarization refractoriness. Adapting the pacing current to the rate-dependent TC the normal relation between VERP and APD was restored: no postrepolarization refractoriness. 
Therefore we conclude that after procainamide, rate-dependent TC increase is of major importance for the phenomenon of postrepolarization refractoriness.

\section{References}

1. Holfman BF, Cranefield PF, Lepeschkin E, et al. Comparison of cardiac monophasic action potentials recorded by intracellular and suction electrodes. Am J Physiol 1959; 196:1297-1301.

2. Korsgren $M$, Leskinen $E$, Sjöstrand $U$. Intracardiac recording of monophasic action potentials in the human heart. Scand J Clin Lab Invest 1966; 18:561-564

3. Franz MR. Long-term recording of monophasic action potentials from human endocardium. Am J Cardiol 1983; 51:1629-1634.

4. Yuan S, Blomström-Lundquist C, Olsson SB. Monophasic action potentials: concepts to practjcal applications. J Cardiovasc Electrophysiol 1994; 5:287-308.

5. Costard-Jaeckle A, Franz MR. Frequency-dependent antiarrhythmic drug eflects on postrepolarization refractoriness and ventricular conduction time in canine ventricular myocardium invivo. J Pharmacol Exp Ther 1989; 251:39-46.

6. Nademanee K, Stevenson WG, Weiss JN, et al. Frequency-dependent effects of quinidine on the ventricular action potential and QRS duration in humans. Circulation 1990; 81:790-796.

7. Franz MR, Chin MC, Sharkey $\mathrm{HR}$, el al. A new single catheter technique for simullaneous measurement of action-potential duration and refractory period in-vivo. J Am Coll Cardiol 1990; 16:878-886.

8. I.azzara R, El-Sherif N, Scherlag BJ. Disorders of cellular electrophysiology produced by ischemia of the canine His bundle. Circ Res $1975 ; 36: 444-454$.

9. Davidenko JM, Antzelevitch C. Electrophysiological mechanisms underlying rate-dependent changes of refractoriness in normal and segmentally depressed canine Purkinje libers. The characteristics of post-repolarization refractoriness. Circ Res 1986; 58:257-268.

10. Camardo JS, Greenspan AM, Horowitz LN, el al Strenglh-interval relation in the human ventricle: effect of procainamide. Am J Cardiol 1980; 45:856-860.

11. Franz MR, Costard A. Frequency-dependent effects of quinidine on the relationship between action-potential cluration and refractoriness in the canine heart in situ. Circulation 1988; 77:1177-1184

12. Nakaya $Y$, Nii H, Nomura $M$, et al. Eflects of lidocaine and quinidine on post-repolarization refractoriness after the basic and premature action potentials: Consideration of aim of antiarrhythmic drug therapy. An Heart J 1989; 118:907-912.

13. Lee RJ, Liem LB, Cuhen TJ, et al. Relation between repolarization and reliactoriness in the human ventricle: Cycle-length dependence and elfect of procainamide. J Am Coll Cardiol 1992; 19:614-618.

14. Janse MJ, van der Steen ABM, van Dam RT, et al. Refractory period of the dog's ventricular myocardium following sudden changes in frequency. Circ Res 1969; 24:251-262

15. Greenspan AM, Camardo JS, Horowitz L.N, et al. Human ventricular refractoriness: effects of increasing current. Am J Cardiol 1981; 47:244-250. 
16. Morady F, Kadish AH, Kushner JA, et al. Comparison of ventricular refractory periods determined by incremental and decremental scanning of an extrastimulus. PACE 1989; 12(P.. I):546554.

17. Morady F, Kadish AH, Rosenheck S, et al. Effect of the intertrain pause on the ventricular effective refractory period measured by the extrastimulus technique. PACE 1990; 13:405-409.

18. Kadish $\mathrm{AH}$, Kou WH, Schmaltz $S$, et al. Effect of atrioventricular relationship on ventricular refractoriness in humans. Am J Physiol 1990; 259:H1463-H1470.

19. Kadish AH, Schmaltz S, Morady F Variability in the measurement of human ventricular refractoriness. PACE 1991; 14:1393-1401.

20. Langberg JL, Calkins $\mathrm{H}$, Sousa J, et al. Effects of drive train stimulus intensity on ventricular refractoriness in humans. Circulation 1991; 84:181-187.

21. Leerssen HM, Vos MA, den Dulk $\mathrm{K}$, et al. Is the ventricular effective refractory period different when determined by incremental versus decremental scanning?: The effect of pacing cycle length, d-sotalol and leveromakalim. PACE 1994; 17(Pt. 11):2084-2089

22. Scherlag BJ, Kosowsky BD, Damato AN. A technique for ventricular pacing from the His bundle in the intact heart. J Appl Physiol 1967; 22:584-587.

23. Kus $T$, Derakhchan $K$, Bouchard $C$, et al. Effects of procainamide on refractoriness, conduction, and excitable gap in canine atrial reentrant tachycardia. PACE 1991; 14(Pt. II);1707-1713.

24. Ware DL, Atkinson JB, Brooks MJ, et al. Ventricular defibrillation in canines with chronic infarction, and eflects of lidocaine and procainamide. PACE 1993; 16:337-346.

25. Furman S, Hurzeler P, Mehra R. Cardiac pacing and pacemakers IV. Threshold of cardiac stimulation. Am Heart J 1977; 94:115-124.

26. Koller BS, Karasik PE, Solomon AJ, et al. Relation between repolarization and refractoriness during programmed electrical stimulation in the human right ventricle. Circulation 1995; $91: 2378$ 2384.

27. Costard-Jaeckle A, Liem L.B. Franz MR. Frequency-dependent effect of quinidine, mexiletine, and their combination on postrepolarization refractoriness in-vivo. J Cardiovasc Pharnacol $1989 ; 14: 810-817$

28. Hook BG, Perlman RL, Callans DJ, el al. Acute and chronic cycle-length dependent increase in ventricular pacing threshold. PACE 1992; 15(Pt. 1):1437-1444.

29. Kastor JA, Josephson ME, Guss SB, el al. Human ventricular refractoriness II. Effects of procainamide. Circulation 1977; 56:462-467.

30. Edvardsson N, Hirsch I, Olsson SB. Acute elfects of lignocaine, procainamide, metoprolol, digoxin and atropine on human myocardial refractoriness. Cardiovasc Res 1984; 18:463-470.

31. Barold SS, McVenes R, Stokes K. Effect of drugs on pacing threshold in man and canines: old and new facts. In SS Bardd, J Mugica (eds.): New perspectives in cardiac pacing. Vol. 3, Moum Kisco, NY, Futura Publishing Company, Inc, 1993, pp. 57-83.

32. Woske $\mathrm{H}$, Belford J, Fastier FN, et al. The effect of procaine amide on excitability, refractoriness and conduction in the mammalian heart. J Pharmacol Exp Ther 1953; 107:134-140.

33. Gay RJ, Brown DF Pacemaker failure due to procainamide toxicity. Am J Cardiol 1974; 34 :728732.

34. Mehta J, Khan AH. Pacemaker Wenckebach phenomenon due to antiarrhythmic drug toxicity. Cardiology 1976; 61:189-194. 
35. Marchlinski FE, Shinnar M. Cycle-length dependent effects of procainamide on ventricular refractoriness. (abstract) J Am Coll Cardiol 1987; 9:227A.

36. Joyner RW, Ramza BM, Osaka $T$, et al. Cellular mechanism of delayed recovery of excitability in ventricular tissue. Am J Physiol 1991; 260:H225-H233.

37. Whalley DW, Wendt DJ, Grant AO. Basic concepts in cellular cardiac electrophysiology: Part I: Ion channels, membrane currents, and the action potential. PACE 1995; 18:1556-1574.

38. Verduyn SC, Vos MA, van der Zande J, et al. Biventricular hypertrophy facilitates occurrence of acquired torsade-de-pointes arrhythmias in dogs. (abstract) Circulation 1995; 92(1):504

39. Kay GN, Mulholland DH, Epstein AE, et al. Effect of pacing rate on the human atrial strengthduration curve. J Am Coll Cardiol 1990; 15:1618-1623.

40. Hoffman BE, Cranefield PF. Electrophysiology of the heart. New York, McGraw-Hill, 1960, pp. $211-256$.

41. Shimizu $W$, Ohe $T$, Kurita $T$, et al. Early afterdepolarizations induced by isoproterenol in patients with congenital long QT syndrome. Circulation 1991; 84:1915-1923.

42. Verduyn SC, Vos MA, van der Zande J, et al. Role of interventricular dispersion of repolarization in acquired torsade-de-pointes arrhythmias: reversal by magnesium. Cardiovasc Res 1997: 34:453-463.

43. Antzelevitch C, Sicouri S, Lukas A, et al. Regional differences in the electrophysiology of ventricular cells: physiological and clinical implications. In MD Zipes, J Jalife (eds.): Cardiac electrophysiology. From cell to bedside, Philadelphia, W. B. Saunders Company, 1995, pp. 228-245. 
[94] 


\title{
Chapter 7
}

\section{High Uniformity of Left and Right Ventricular Repolarization Dynamics Induced by an Abrupt Decrease in Pacing Cycle Length in a Dog is not Affected by Left-Ventricular Ischemia}

\author{
Hendrik M. Leerssen, Marc A. Vos, Richard Houben, ${ }^{*}$ Karel den Dulk, and \\ Hein J.J. Wellens. \\ Department of Cardiology, Cardiovascular Research Institute Maastricht, Maastricht \\ University, The Netherlands, and *Medtronic Bakken Research Center, Maastricht, \\ The Netherlands. \\ Published in I Cardiovasc Electrophysiol 2000; 11:421-429. \\ Editorial by Michiel J. Janse. \\ Published in $」$ Cardiovasc Electrophysiol 2000; 11:430-431.
}




\section{Abstract}

Introduction: After an abrupt increase in heart rate action-potential duration (APD) will shorten. To assess the effect of ischemia on APD shortening dynamics we compared right ventricular (RV) and left-ventricular (LV) APD shortening induced by an abrupt decrease in pacing cycle length (PCL) during control and LV ischemia.

Methods and Results: In eight anesthetized dogs with atrioventricular block, endocardial LV and RV APD were determined simultaneously after an abrupt PCL decrease from 800 to $350 \mathrm{msec}$. Measurements were repeated during left anterior descending coronary artery (LAD) occlusion. During control, LV and RV APD shortened $97 \pm 27$ and $71 \pm 14 \mathrm{msec}$, respectively $(\mathrm{P}<0.05)$. Shortening was pronounced in a short initial phase and gradual in the longer secondary phase. Linear regression analysis revealed very high uniformity of LV and RV APD shortening dynamics $\left(r^{2}=0.96 \pm 0.01\right)$. During repeated LAD occlusion, ischemia induced a gradual LV APD shortening from $314 \pm 25$ to a new steady-state value of $251 \pm 23$ msec, whereas RV APD remained stable at $289 \pm 28 \mathrm{msec}$. The additional PCL decrease resulted in LV and RV APD shortening of $72 \pm 8$ and $68 \pm 15 \mathrm{msec}$, respectively, with the same high uniformity of shortening dynamics as during control $\left(r^{2}=0.94 \pm 0.03\right)$.

Conclusion: There is a pronounced difference in APD shortening dynamics induced by an abrupt decrease in PCL compared with ischemia. LV shortening dynamics induced by a decrease in PCL are not affected by LV ischemia, preserving a high interventricular uniformity of repolarization dynamics. 


\section{Introduction}

The duration of repolarization or refractoriness at steady-state paced heart rates has been studied extensively in-vivo. Depending on the model and the conditions involved, investigators determined the ventricular effective refractory period (VERP), 1,2 action-potential duration (APD), ${ }^{2-6}$ activation recovery intervals, ${ }^{7.8}$ stimulus-T interval (STI), ${ }^{9}$ or a combination of these variables.

The dynamic behavior of repolarization and refractoriness after the onset of ventricular tachycardia (VT) is more difficult to study. Investigators used VERP, ${ }^{1}{ }^{10}$ APD,, $10-12$ and $\mathrm{QT}^{13}$ to describe this dynamic behavior, often simulating VT by an abrupt decrease in pacing cycle length ( $P C L)$.

In a normally perfused heart, we showed by simultaneous recordings of VERP, APD and STI in the right ventricle (RV) that, although absolute values of the start and end points are different, these parameters shorten with a remarkably high uniformity. ${ }^{1+}$ The dynamics of this process are characterized by a very short initial phase with pronounced shortening and a secondary phase with more gradual shortening toward the new steady-state value. Using a bi-exponential mathematical model, this behavior can be described accurately. ${ }^{1+}$

Until now, little has been known about the effect of ischemia on repolarization dynamics. ${ }^{11}$ Comparison between left-ventricular (LV) and RV repolarization dynamics during left-sided ischemia has never been made. We investigated the effect of LV ischemia on LV and RV repolarization dynamics using endocardially placed monophasic action-potential (MAP) catheters. The experiments were performed in exposed hearts of anesthetized dogs with complete atrioventricular (AV) block.

\section{Methods}

Animal handling was in accordance with the Dutch Law on Animal Experimentation and the European Directive for the Protection of Vertebrae Animals used for Experimental and other Scientific Purposes (86/609/EU). The experiments were approved by the Committee for Experiments on Animals of the Maastricht University.

\section{General Information}

Experiments were performed in eight anesthetized mongrel dogs with induced complete AV block (weight: $27 \pm 7 \mathrm{~kg}$, (range $21-41$ )). ${ }^{15}$ Dogs were ordered through a licensed dealer and selected on the basis of body weight and age (adult), not on gender or race. At the end of the experiments, the dogs were sacrificed.

Anesthesia was induced after overnight fasting by (1) intramuscular premedication $(1 \mathrm{ml} / 5 \mathrm{~kg}$ : $10 \mathrm{mg}$ oxycodon $\mathrm{HCl}, 1 \mathrm{mg}$ acepromazine, and $0.5 \mathrm{mg}$ 
atropine sulphate) and (2) intravenous sodium pentobarbital (20 mg/kg, Nembutal, Sanofi Sante, Maassluis, The Netherlands). The dogs were artificially ventilated (Pulmonat, Dräger, Lübeck, Germany), through a cuffed endotracheal tube at a respiratory rate of 12 to 14 per minute using a mixture of $40 \%$ oxygen, $60 \%$ nitrous oxide, and halothane (vapor concentration $0.5 \%$ to $1 \%$ ). Tidal volume was adjusted (10 to $25 \mathrm{ml} / \mathrm{kg}$ ) to maintain an end-expired carbon dioxide concentration between 3.5 and $4 \%$. A thermal mattress was used to maintain adequate body temperature. During the experiment, the dog received 0.5 to 1 liter $0.9 \% \mathrm{NaCl}$ through the cephalic or saphenous vein to prevent volume depletion.

The left anterior descending coronary artery (LAD) was occluded 1.5 to $2 \mathrm{~cm}$ below the auricle of the left atrium (under the first or second side branch of the LAD) using a metal bulldog clip, which allowed precise timing of LAD occlusion and release.

The first MAP catheter (Franz MAP-Pacing catheter, EP Technologies Inc., Mountain View, CA, USA) was introduced via the jugular or femoral vein and positioned under fluoroscopic guidance against the endocardium of the RV apex. The second MAP catheter was introduced via the carotid artery and positioned in the perfusion area of the LAD. The position was accepted if LV APD shortened at least $35 \mathrm{msec}$ during occlusion. The MAP signals were amplified with a custombuild DC coupled amplifier having a $20 \mathrm{mV}$ calibration pulse. The offset of this amplifier was adjusted to the recorded signal. The minimum amplitude for acceptance of the signal was $15 \mathrm{mV}$.

The output strength of the stimulator was determined with $0.1 \mathrm{~mA}$ precision and adjusted to twice the diastolic threshold determined at $800 \mathrm{msec}$ PCL. All signals and markers were sampled at a rate of $1 \mathrm{kHz}$ and stored on a hard disk.

To determine the time course of ischemia induced APD shortening, pilot studies were performed using 30 minutes LAD occlusions. Because no clear additional APD shortening was found after 4 minutes of ischemia, 6 minutes ischemia episodes were selected to combine the effect of ischemia with the rate switch.

\section{Experimental Protocol}

Abrupt $P C L$ decrease: From a steady state condition at $P C L=800 \mathrm{msec}$, the PCL was abruptly decreased to $350 \mathrm{msec}$ for 5 minutes before returning to 800 msec (Figure 7.1, 5 - $10 \mathrm{~min}$ ).

First and second LAD occlusions: The duration of the first and second LAD occlusions was 6 minutes. Between occlusions, reperfusion was allowed for 24 minutes (Figure 7.1, $1^{\text {sl }}$ LAD occlusion: 15 - 21 min, $2^{\text {nd }}$ LAD occlusion: 45 $51 \mathrm{~min}) .3,+, 16,17$

Third LAD occlusion + abrupt PCL decrease: The duration of the third occlusion was 6 minutes. During the first 4 min the PCL remained at 800 msec for the first 4 minutes, then was abruptly decreased to $350 \mathrm{msec}$ for the remaining $2 \mathrm{~min}$ utes (Figure 7.1, $75-81 \mathrm{~min}$ ). 


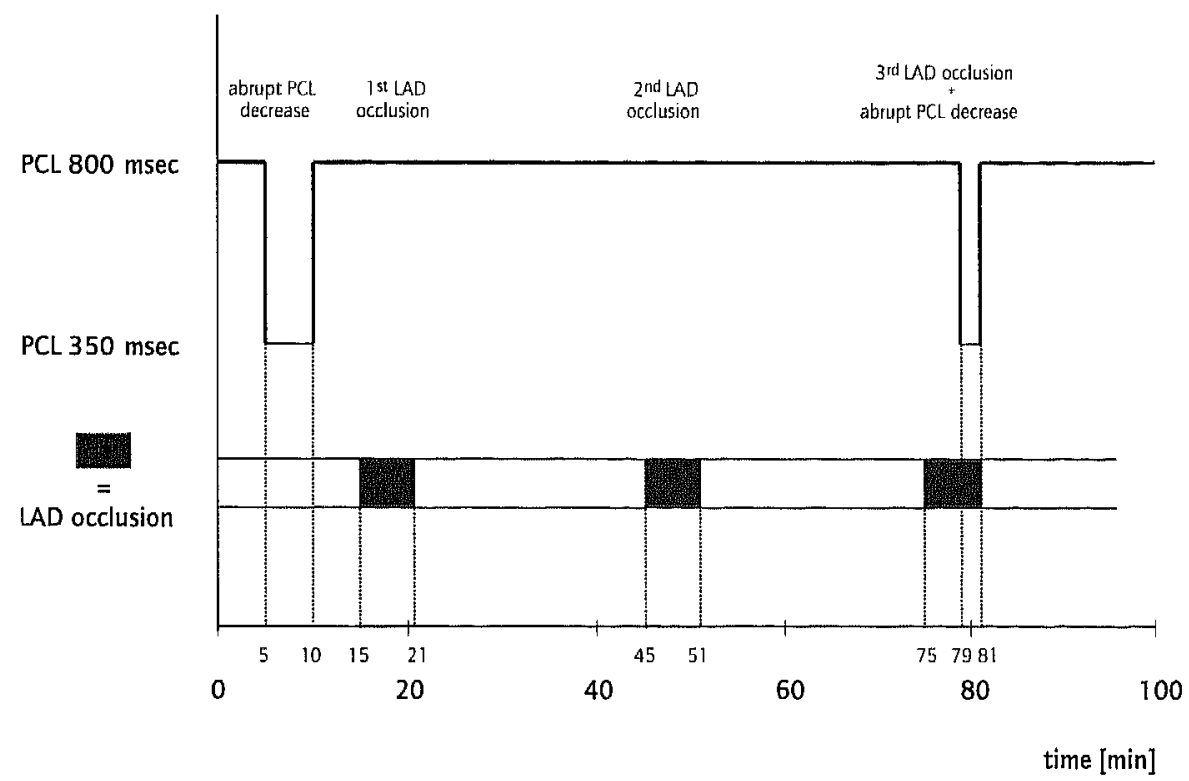

Figure 7.1 - Experimental protocol.

The abscissa indicates time in minutes. Episodes of PCL changes are indicated on the ordinate and LAD occlusions are visualized by black bars. See Methods for details. Abbreviations: PCL = pacing cycle length; $L A D=$ left: anterior descending coronary artery.

\section{Data Analysis}

Phases of the MAP were defined according to the definitions used for transmembrane potentials, i.e., amplitude as the voltage difference between phases 2 and 4 of the MAP signal. ${ }^{18}$ The duration was defined as the interval from the beginning of the upstroke of the local activation to the end of repolarization (APD100, Figure 7.2). APD was determined via ofl-line analysis using "ECGview" software (developed in collaboration with the Department of Medical Technology, Maastricht University, Maastricht, The Netherlands). The settings of this software allowed APD determination with 5 msec accuracy.

Using nonlinear regression analysis a bi-exponential model was used $\left(y=c_{0}+c_{1} \exp \left(-t / \tau_{1}\right)+c_{2} \exp \left(-t / \tau_{2}\right)\right)$ to fit the dynamics of LV and RV APD shortening after the abrupt decrease of PCL from 800 to $350 \mathrm{msec}$. In this model $y=A P D$ and $t=$ time expressed in beats of $350 \mathrm{msec}$ PCL. For a detailed description of this model we refer to chapter $5.1+4$

\section{Three Dimensional Plots}

In addition to APD shortening (Figure 7.3), ischemia is associated with marked changes in MAP morphology. These changes can be visualized with threedimensional plots (Figure 7.4, Medtronic Bakken Research Center, Maastricht, 


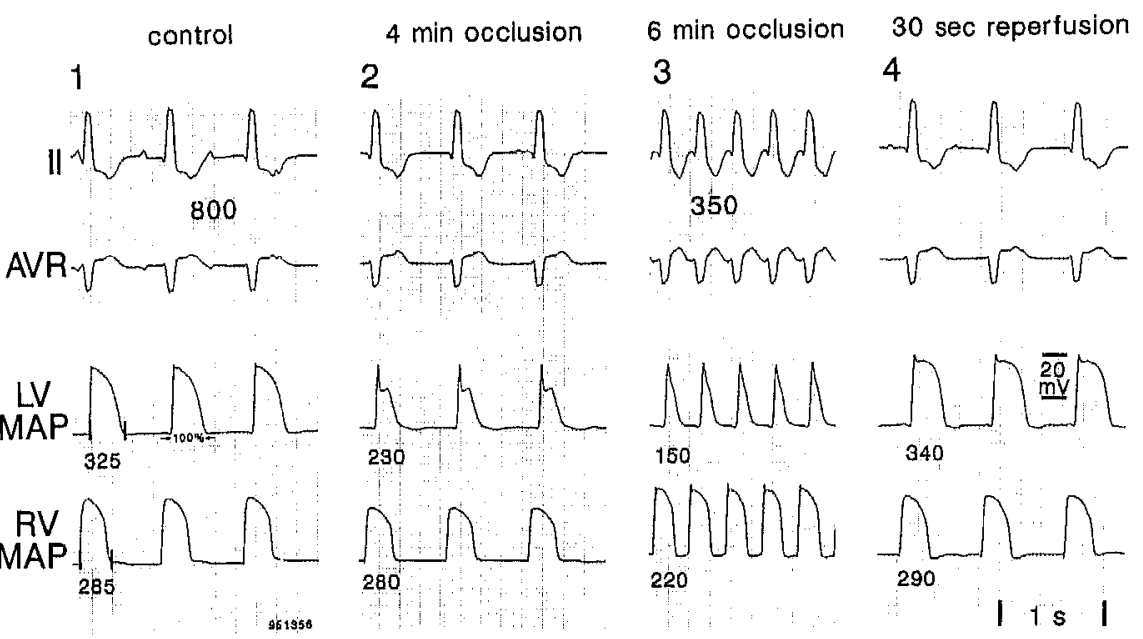

Figure 7.2 - ECGs and monophasic action potentials (MAPs) during the experiment.

Two ECC leads (II and AVR) together with IV and RV MAP signal are shown at a paper speed of $25 \mathrm{~mm} / \mathrm{sec}$. Panel 1 shows the control situation with RV pacing at pacing cycle length $800 \mathrm{msec}$ in this chronic AV block $\operatorname{dog}$. APD100 is the interval from the beginning of the upstroke of the local activiation to the end of repolarization as measured in the MAP recording (see panel 1). The LV APD is longer than the RV APD, as has been described in this animal model. The third LAD occlusion (panel 2) causes $\mathrm{LV}$ ischemia that results in maximal shortening of the LV APD at 4 minutes. At this time, the abrupt decrease in pacing cycle length from 800 to $350 \mathrm{msec}$ is added, leading to marked APD shortening of both ventricles (panel 3). Shortly after reperfusion $(30 \mathrm{sec}$ ) the APDs retum to baseline (panel 4). Abbreviations: IV = left-ventricular; RV = right ventricular; MAP $=$ monophasic action potential; $A V=$ atrioventricular; $A P D=$ action-potential duration; $L A D=$ left anterior descending coronary artery.

The Netherlands). To configure these plots, the maximum slope of RV MAP upstroke was used as a reference point for extraction of MAP signals. The baseline before the upstroke was corrected by subtraction of the mean amplitude over an interval of $10 \mathrm{msec}$ in the silent region. Valid MAPs were automatically identified by calculating the correlation coefficient between the first valid MAP, manually identified by an expert, and all subsequent MAPs. The included MAPs were time aligned according to the positive or negative maximum covariance indexes.

\section{Statistics}

Primer of Biostatistics (McGraw-Hill Inc., New York, NY, USA) was used for statistical tests and SPSS for Windows (SAS Institute, Cary, NC, USA) to fit the bi-exponential model. $\mathrm{P}<0.05$ was considered statistically significant.

APD shortening induced by the PCL decrease during control and LAD occlusion: Uniformity of LV and RV APD shortening dynamics was quantified using linear regression analysis. Results of the fit of the bi-exponential model for LV and RV APD and of the measured amount of LV and RV APD shortening were compared using paired $t$-tests. 

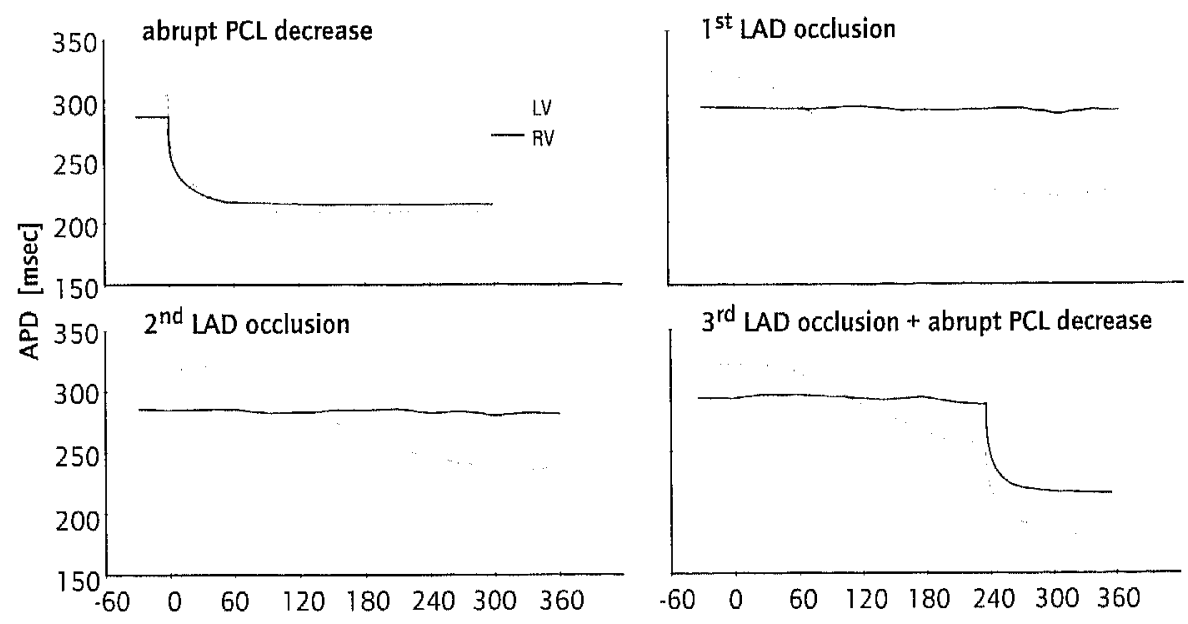

time [sec]

Figure 7.3 - Action-potential durations (APDs) during the experiment.

$t=0$ sec indicates the onset of PCL. decrease from 800 to 350 msec or the start of the LAD occlusion. Data are presented as the mean of individual experiments. "Abrupt $\mathrm{PCL}$ decrease" panel = results of nonlinear regression analysis of the bi-exponential model for LV and RV. " 1 st LAD occlusion" and "2 $2^{\text {nd }}$ LAD occlusion" panels = LV and RV APD behavior during the first and second LAD occlusion, at $800 \mathrm{msec} P C L$. "3 $3^{\text {rd }}$ LAD occlusion + abrupt $\mathrm{PCL}$ decrease" panel $=\mathrm{LV}$ and RV APD behavior during the third LAD occlusion with the abrupt PCL. decrease from 800 to 350 msec after 4 minutes. Abbreviations: $P C L=$ pacing cycle length; $L A D=$ left anterior descending coronary artery; $L V=$ left ventricle; $R V=$ right ventricle; $A P D=$ action-potential duration.

APD shortening induced by the LAD occlusions: LV APD shortening at 60 , 120,240 and 360 seconds was compared with the value at $t=0 \mathrm{sec}$ using repeated measures ANOVA followed by Bonferroni t-tests.

\section{Results}

\section{Abrupt PCL Decrease}

There is a different starting point for the two ventricles: LV APD at 800 msec PCL is longer than RV APD ( $305 \pm 31$ vs $287 \pm 27 \mathrm{msec} ; \mathrm{P}<0.05)$. As a result of the PCL decrease to $350 \mathrm{msec}$, LV APD shortens significantly more than RV APD (97 \pm 27 vs $71 \pm 14 \mathrm{msec} ; \mathrm{P}<0.05)$. This results in a similar value for $L V$ and RV APD at $350 \mathrm{msec}$ PCL steady-state circumstances (Table 7.1 and Figure 7.3: abrupt PCL decrease). The parameters $c_{1}$ and $c_{2}$ of the bi-exponential model show that this additional I.V APD shortening takes place during both exponential phases (Table 7.1). Comparing the total range of LV and RV APD shortening dynamics, there is a high interventricular uniformity (linear regression analysis: $\mathrm{r}^{2}=0.96$ \pm 0.01 ). 

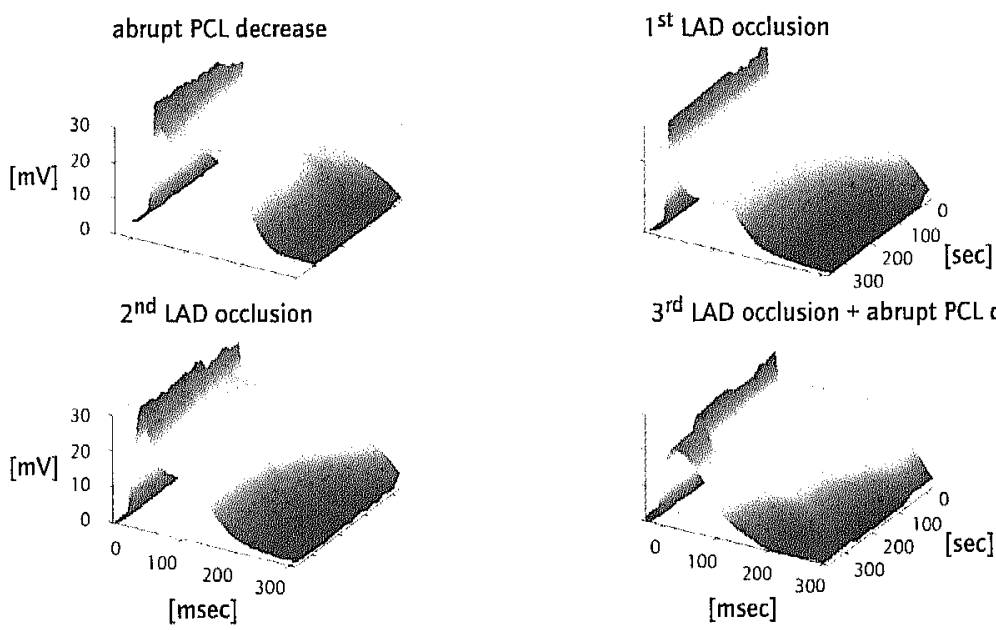

$3^{\text {rd }}$ LAD occlusion + abrupt PCL decrease

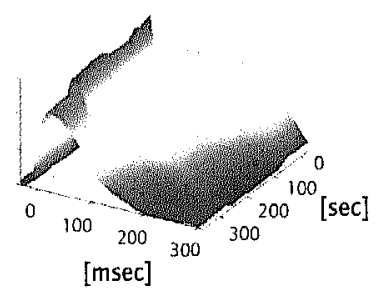

Figure 7.4 - Three dimensional representation of the monophasic action-potential (MAP) changes in the left ventricle (LV) in a single representative dog.

The time scale in sec indicates the relative time in the protocol, $t=0$ sec indicates the moment of the abrupt PCL decrease or the start of the LAD occlusion. The time scale in msec indicates APD. The vertical scale (in mV) indicates the relative amplitude of the MAP signal with baseline level set to $0 \mathrm{mV}$. Morphology and APD changes induced by the abrupt PCL decrease are clearly distinct from changes induced by ischemia (See Three Dimensional Plots in the Methods section for details). Abbreviations: MAP = monophasic action potential; $L V=$ left ventricle; $P C L=$ pacing cycle length; $L A D=$ left anterior descending coronary artery; $A P D=$ action-potential duration.

Table 7.1 Shortening dynamics of APD after an abrupt decrease in $\mathrm{PCL}$ described by the biexponential model.

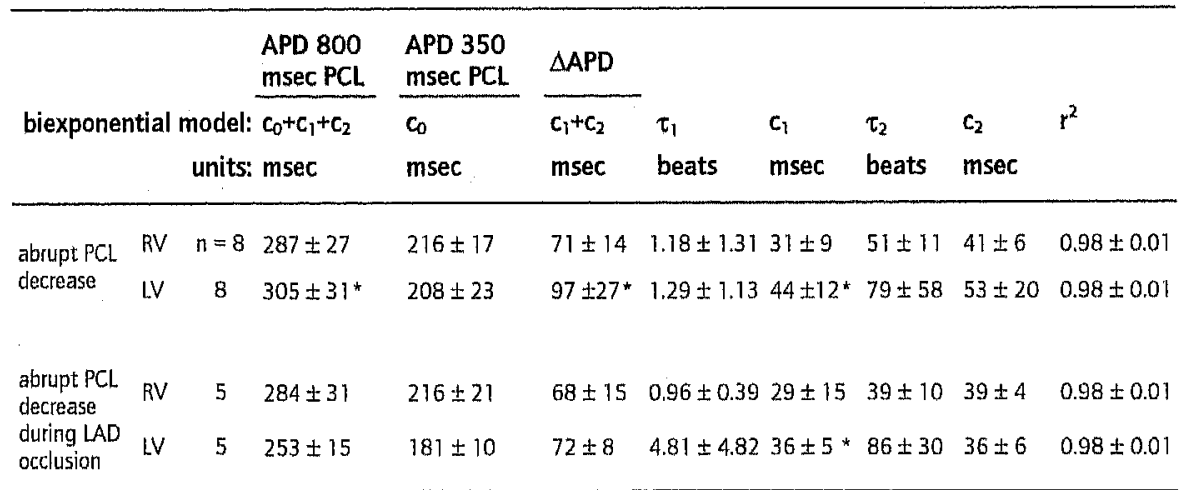

Abbreviations: $A P D=$ action-potential duration; $P C L=$ pacing cycle length; $L V=$ left ventricle; $R V=$ right ventricle. Biexponential model: $y=c_{0}+c_{1} \exp \left(-t / \tau_{1}\right)+c_{2} \exp \left(-t / \tau_{2}\right)$. In this model y represents LV or RV APD; $t$ represents time, expressed in beats with a cycle length of $350 \mathrm{msec}$. The table shows APD steady-state values at $800\left(c_{0}+c_{1}+c_{2}\right)$ and $350 \mathrm{msec} P C L\left(c_{0}\right)$. The total amount of shortening after the abrupt PCL decrease from 800 to $350 \mathrm{msec}(\triangle \mathrm{APD})$ is indicated by $c_{1}+c_{2}, \tau_{1}$ And $\tau_{2}$ are the time constants of the first and second exponential phase, expressed in beats with $350 \mathrm{msec} P C L$. The amount of shortening during this first and second exponential component is expressed by $c_{1}$ and $c_{2} . r^{2}$ is the coefficient of determination. Significant difference between the fitting parameters of $\mathrm{LV}$ with RV APD is indicated by * (paired $t$ test, $\mathrm{P}<0.05$ ). All values are expressed as mean \pm SD. For a detailed description of the biexponential model we refer to chapter 5 of this thesis. 
First and Second LAD Occlusions

A representative example of the induced electrophysiological changes that occur during ischemia is shown in Figure 7.2, panel 2. The effect of the first and the second LAD occlusions on LV and RV APD is shown in Figure 7.3. As expected, LAD occlusion had no effect on RV APD (Figure 7.2, panel 2 and Figure 7.3). The mean difference in APD between 0 and 6 minutes is $3 \pm 7 \mathrm{msec}$. There is clear LV APD shortening as a result of ischemia, which becomes significant after 120 or 240 seconds depending on the protocol (Table 7.2 and Figure 7.3). As anticipated from the pilot experiments, there is no additional APD shortening during the last 2 minutes of the 6 minute occlusion period (Table 7.2).

Table 7.2 The effect of LAD occlusion on LV APD at 800 msec PCL,

\begin{tabular}{llll}
\hline & \multicolumn{2}{l}{ LV APD (msec) } & \\
\cline { 2 - 4 } $\mathrm{t}(\mathrm{sec})$ & first occlusion $(n=7)$ & second occlusion $(n=7)$ & third occlusion $(n=7)$ \\
\hline 0 & $314 \pm 36$ & $318 \pm 36$ & $314 \pm 25$ \\
60 & $293 \pm 31$ & $317 \pm 32$ & $311 \pm 23$ \\
120 & $259 \pm 43^{*}$ & $288 \pm 27$ & $286 \pm 18 *$ \\
240 & $224 \pm 36 *$ & $249 \pm 22 *$ & $251 \pm 23 *$ \\
360 & $225 \pm 31 *$ & $241 \pm 22 *$ & \\
\hline
\end{tabular}

Abbreviations: $L A D=$ left anterior descending coronary artery; IV APD = left ventricular action potential duration. $t=0$ indicates the moment of LAD occlusion; $t=60,120,240$, and 360 sec indicates the duration of LAD occlusion. LV APD At $360 \mathrm{sec}$ after the third LAD occlusion is not included because the pacing cycle length decreased to $350 \mathrm{msec}$ after $240 \mathrm{sec}$. Significant difference comparing the value of LV APD at $t=0$ sec with $t=60,120,240$ and $360 \mathrm{sec}$ is indicated by * (repeated measures of ANOVA followed by Bonferroni $t$ tests, $P<0.05$ ).

Comparison of Abrupt PCL Decrease and LAD Occlusion

Shortening dynamics of APD induced by an abrupt decrease in PCL are quite distinct from those induced by ischemia. The abrupt decrease in PCL from 800 to $350 \mathrm{msec}$ induces a biphasic response with immediate and pronounced shortening in the initial phase and a secondary phase with more gradual shortening towards the new steady-state value, which will be reached alter approximately 250 beats or 90 seconds (Figure 7.3: abrupt PCL decrease). The onset of shortening after ischemia is often delayed. Furthermore it is much slower and reaches steady-state after approximately 4 minutes (Figure 7.3: $1^{\text {st }}$ and $2^{\text {nd }}$ LAD occlusion).

Third LAD Occlusion + Abrupt PCL Decrease

Ischemia-induced LV APD shortening reaches significance after $120 \mathrm{sec}-$ onds (Table 7.2). The abrupt PCL decrease at 4 minutes of ischemia induces an 
immediate LV and RV APD shortening (Figure 7.2, panel 3 and Figure 7.3: 3 rd LAD occlusion + abrupt PCL decrease). Although ischemia shortens LV APD from $314 \pm 25 \mathrm{msec}$ to $251 \pm 23 \mathrm{msec}$, the uniformity of LV and RV APD shortening dynamics induced by the PCL decrease is not affected (linear regression analysis: $r^{2}=0.94 \pm 0.03$ ). Fitting the bi-exponential model, the results show that the total amount of shortening induced by the abrupt PCL decrease (Table 7.1: $\triangle \mathrm{APD}$, $\left.c_{1}+c_{2}\right)$ is not different for the ventricles. Complete LV and RV APD recovery was achieved before 1 minute of reperfusion (Figure 7.2, panel 4). Although LV ischemia already shortens LV APD before the abrupt PCL decrease, the uniformity of LV and RV APD shortening dynamics induced by the PCL decrease is as high as during control. In other words, partial ischemia of the LV does not influence the shortening dynamics induced by an abrupt PCL decrease.

MAP Morphology Changes

Clear differences can be found when comparing the dynamics of LV APD shortening induced by an abrupt $\mathrm{PCL}$ decrease and ischemia. Additional differences can be found when analyzing the changes of LV MAP morphology in response to these two triggers (Figure 7.4).

Abrupt PCL decrease: After the abrupt PCL decrease, the slope of phase 1 gradually becomes less steep. This is accompanied by an increase in amplitude of the plateau phase. This process starts around the time the new steady-state value of APD has been reached (Figure 7.4).

LAD occlusions: In response to ischemia the slope of phase 1 becomes less steep, and there is a decrease in the amplitude of the plateau phase (Figure 7.2 and Figure 7.4).

LAD occlusion + abrupt PCL decrease: The MAP morphology changes induced by the combination of ischemia and an abrupt PCL decrease are a combination of the separate responses (Figure 7.4).

\section{Discussion}

The most important findings of this study can be summarized as follows. (1) There is a high interventricular uniformity of APD shortening dynamics in response to an abrupt decrease in PCL. (2) The response of APD and MAP morphology induced by an abrupt decrease in PCL is clearly different from the response induced by ischemia. (3) The high interventricular uniformity of APD shortening dynamics induced by an abrupt $\mathrm{PCL}$ decrease is not affected by $\mathrm{LV}$ ischemia.

Methodological Considerations

With regard to the methodology, numerous decisions had to be made. To quantify the duration of repolarization, APD registered by a MAP catheter was 
chosen instead of VERP or STI. Considering the time consuming protocol necessary for VERP determination ${ }^{1,14}$ and the possible development of post repolarization refractoriness under the given circumstances 19,20 VERP was no option. Although the ability of STI to monitor ischemia has been described, ${ }^{21}$ APD can be considered the gold standard since it has been studied extensively in humans, ${ }^{4,22}$ dogs, $, 3,7,16,23-25$ and in pigs. $5,8,26,27$

The decision to use AV block dogs was based on the fact that bradycardia will lead to long values for baseline APD 28 and allows PCL changes of sufficient magnitude.

Although it is known that the effect of ischemia on epicardial APD shortening is more pronounced than on endocardial APD shortening, ${ }_{1}^{17,23,24}$ endocardial lead positions were chosen to mimic the lead positions of implantable devices.

To avoid intra-individual variation between subsequent LAD occlusions in dogs, changes in collateral flow during repeated episodes of ischemia and infarction had to be avoided. 17,29 Furthermore, the electrophysiological changes had to be completely reversible. This can be accomplished by 4- to 6-minute LAD occlusions followed by 24 minutes of reperfusion. $3,4,16,17,30$ The reproducibility of LV APD values before each LAD occlusion (Table 7.2) confirms that the induced changes are completely reversible.

Even during a 30 minute LAD occlusion in pilot studies, we found that LV APD reached its new steady-state value within 4 minutes, with no further drift toward shorter values. Therefore, PCL decrease during ischemia was started after 4 minutes of ischemia. During the remaining 2 minutes the steady-state value of the shorter PCL will be reached. ${ }^{14}$ In pig models, ischemia affects the duration of repolarization differently. During ischemia, APD continued to shorten until there was complete ischemic degeneration. ${ }^{5,27}$ This difference in response probably is caused by the absence of collaterals in pigs.

It is known from the literature that there is a difference in the electrophysiological response between the first and the following short-lasting ischemic episodes. Investigators show that not only the amount but also the speed of the electrophysiological changes are reduced. $3,16,23$ Therefore, we used the second LAD occlusion as control. Furthermore, comparison of the response described in this article and the response after additional interventions, such as the administration of antiarrhythmic drugs, can only be made if a preconditioning effect can be excluded.

APD Shortening Induced by Abrupt PCL Decrease and Ischemia

Baseline LV APD in dogs is longer than RV APD due to intrinsic electrophysiological differences between the ventricles. ${ }^{31}$ These differences become more pronounced with severe bradycardia and as a result of several remodeling processes that occur after AV block. ${ }^{28}$ Therefore, the longer APD in the LV was anticipated.

We previously showed in the same animal model that RV APD shortening after an abrupt decrease in PCL is biphasic: a very short initial phase with imme- 
diate and pronounced shortening and a secondary phase with more gradual shortening toward the new steady-state value. Using a bi-exponential mathematical model, this biphasic behavior can be described accurately. ${ }^{14}$ This was recently confirmed in humans. ${ }^{32}$

In this study, we found that LV APD in response to an abrupt decrease in PCL is identical to RV APD response, not only during control but also during ischemia (Figures 7.2 trough 7.4).

Shortening of LV and RV APD induced by a PCL decrease from 800 to 350 msec during control and ischemia was between 68 and $97 \mathrm{msec}$ (Table 7.1). This is well within the range of 6 to $120 \mathrm{msec}$ found by other investigators. ${ }^{6,10-12,14,22}$

In our AV block dog model, LV APD shortening due to the LAD occlusion during steady-state pacing is gradual and sometimes delayed, with an asymptotic [106] approach to its end value after approximately 4 minutes. Other investigators found the same behavior. ${ }^{3,16,17}$ A similar time course in APD shortening was found in ischemic pig and rabbit hearts. ${ }^{26}$ An initial rise in APD during the initial 2 minutes of ischemia followed by shortening has been reported from epicardial APD measurements in dogs and pigs. ${ }^{16,27}$

\section{MAP Morphology Changes Induced by Ischemia}

With regard to ischemic changes on MAP morphology, data in the literature are mainly qualitative. Nevertheless, the changes reported from experiments with endocardial and epicardial contact electrodes are characteristic and include loss of diastolic potential, loss of amplitude, and shortened duration. These data were reported in patients during bypass surgery, in dog models, $3,16,17,23,25$ and in pigs ${ }^{5}$ and are in accordance with our findings (Figure 7.4). Our MAP recording technique using a DC coupled differential amplifier does not allow quantitative assessment of the diastolic potential. Therefore baseline level of the MAP signal was always set to $0 \mathrm{mV}$.

\section{Relation Between APD and APD Shortening}

Baseline LV APD in the AV block dog is long and resembles the situation in the patient. Even after 4 minutes of LAD occlusion, LV APD is long enough to allow the additional shortening induced by the PCL decrease. Therefore, there is no significant decrease in the amount of LV APD shortening after the PCL decrease comparing control with ischemia ( $97 \pm 27$ vs $72 \pm 8$ ). However, after the abrupt PCL decrease during control, LV APD shortens significantly more than RV APD, whereas this difference disappears during ischemia. Therefore, we believe that the amount of APD shortening after an increase in heart rate is related to the baseline APD value. If baseline APD is too short, then APD response will be reduced. This conclusion is supported by Rosenbaum et al. 11 These investigators tested the combined effect of ischemia and PCL decrease in guinea pig hearts with short baseline APDs. They reported a reduction of APD shortening from $22 \mathrm{msec}$ during control to $6 \mathrm{msec}$ during ischemia after a PCL decrease from 500 to 
$300 \mathrm{msec}$. The mono-exponential model used to fit this behavior showed slowing of the shortening dynamics expressed by the increase of the time constant $\tau$.

In our experiments, the amount of LV APD shortening induced by the PCL decrease is not reduced comparing control with ischemia. Therefore the time constants $\tau_{1}$ and $\tau_{2}$ of the bi-exponential model do not change (Table 7.1).

\section{Limitations of the Study}

We studied the dynamics of repolarization in an AV block dog model because this model allows PCL changes of sufficient magnitude. We do not know how the electrophysiological response of this model corresponds with the response in patients in sinus rhythm. However, Denardo and Stevenson ${ }^{33}$ showed that the adaptation of RV refractoriness to an abrupt increase in heart rate is not impaired in patients in chronic heart failure.

To simulate VT, the PCL was decreased from 800 to $350 \mathrm{msec}$. Other values of PCL and PCL change were not tested. Electrophysiological changes during the PCL increase from 350 to $800 \mathrm{msec}$ and the reperfusion phase were not recorded in all animals; therefore, these data are not included in this study.

Although the position of LAD occlusion in each dog was selected to obtain a similar size of the ischemic area, inter-individual variations may have been present. ${ }^{30}$

\section{Clinical Implications}

In earlier studies, we used VERP, APD, and STI to describe RV repolarization dynamics after a simulated VT. ${ }^{14}$ Although there is considerable inter-individual variation in steady-state values and repolarization dynamics, ${ }^{34}$ the intraindividual uniformity of repolarization dynamics described by VERP, APD and STI is high, allowing prediction of the duration of repolarization and refractoriness by either method. In implantable cardioverter defibrillators, STI information can be made available on-line; therefore, STI might be used to determine coupling intervals of premature stimuli in a new and automatic antitachycardia pacing mode..$^{14}$ It is known that VT often originates in the LV under ischemic conditions in patients. This is the first study in which the effect of LV ischemia on the interventricular unilormity of repolarization dynamics is shown. Whether this will allow automatic antitachycardia pacing based on right sided STI information needs to be addressed.

\section{Conclusion}

The electrophysiological response of APD induced by an abrupt decrease in PCL is clearly different from the response induced by ischemia. The high interventricular uniformity of APD shortening dynamics incluced by an abrupt PCL decrease is not affected by LV ischemia. 
Acknowledgements: The authors gratefully acknowledge the contribution of Jolanda van der Zande during the experiments and Ben Vonk (Vitatron Medical B.V., Dieren, The Netherlands) for excellent technical support.

\section{References}

1. Janse MJ, van der Steen ABM, van Dam RT, et al. Refractory period of the dog's ventricular myocardium following sudden changes in lrequency. Circ Res 1969; 24:251-262.

2. Edvardsson N, Hirsch 1 , Olsson SB. Right ventricular monophasic action potentials in healthy young men. PACE 1984; 7(PL. V):813-821.

3. Franz MR, Flaherty JT, Platia EV, et al. Localization of regional myocardial ischemia by recording of monophasic action potentials. Circulation 1984; 69:593-604.

4. Taggart $P$, Sutton $P$, Runnalls $M$, et al. Use of monophasic action-potential recordings during routine coronary-artery bypass surgery as an index of localised myocardial ischaemia. The Lancet 1986; 28:1462-1464.

5. Dilly SG, Lab MJ. Changes in monophasic action-potential duration during the first hour of regional myocardial ischaemia in the anaesthetised pig. Carcliovasc Res 1987; 21:908-915.

6. Franz MR, Swerdlow CD, Liem LB, et al. Cycle-length dependence of human action-potential duration in-vivo. J Clin Invest 1988; 82:972-979.

7. Haws CW, Lux RL. Correlation between in-vivo transmembrane action-potential durations and activation-recovery intervals from clectrograms. Circulation 1990; 81:281-288.

8. Ejima J, Martin D, Engle C, et al. Ability of activation recovery intervals to assess action-potential duration during acute no-flow ischemia in the in situ porcine heart. J Cardiovasc Electrophysiol 1998; 9:832-844.

9. Donaldson RM, Rickards AF Evaluation of drug-induced changes in myocardial repolarisation using the paced evoked response. Br Heart J 1982; 48:381-387.

10. Morgan JM, Cunningham AD, Rowland E. Relationship of the effective refractory period and the monophasic action-potential duration after a step increase in pacing frequency. PACE 1990; 13:1002-1008.

11. Rosenbaum DS, Kaplan DT, Kanai A, et al. Repolarization inhomogeneities in ventricular myocardium change dynamically with abrupt cycle-length shortening. Circulation 1991; 84:1333-1345.

12. Kanaan N, Jenkins J, Childs $\mathrm{K}$, el al. Monophasic action-potential duration during programmed electrical stimulation. PACE 1991; 14:1049-1059.

13. Lau $C P$, Freedman AR, Fleming $S$, et al. Hysteresis of the ventricular paced $Q T$ interval in response to abrupt changes in pacing rate. Cardiovasc Res 1988; 22:67-72

14. Leerssen HM, Vos MA, den Dulk K, et al. Steady-state and dynanic behavior of ventricular repolarization and refractoriness in the dog. PACE 1998; 21:1766-1777.

15. Vos MA, Gorgels APM, Lipcsei GC, et al. Mechanism-specific antiarthythnic elfects of the potassium channel activator levcromakalim against repolarization-dependent tachycardias. J Cardiovasc Electrophysiol 1994; 5:731-742.

16. Russell DC, Snith HJ, Oliver MF. Transmembrane potential changes and ventricular fibrillation during repetitive myocardial ischaemia in the dog. Br Heart J 1979; 42:88-96. 
17. Miyoshi S, Miyazaki T, Moritani K, et al. Dilferent responses of epicardium and endocarclium to $\mathrm{K}_{\text {ATP }}$ channel modulators during regional ischemia. Am J Physiol 1996; 271:H140-H147.

18. Franz MR. Long-term recording of monophasic action potentials from human endocardium. Am J Carcliol 1983; 51:1629-1634.

19. Davidenko JM, Antzelevitch C. Electrophysiological mechanisms underlying rate-dependent changes of refractoriness in normal and segmentally depressed canine Purkinje fibers. The characteristics of post-repolarization refractoriness. Circ Res 1986; 58:257-268.

20. Leerssen HM, Vos MA, den Dulk K, et al. Rale-dependent elfects of procainamide on the threshold current for pacing in the selting of postrepolarization refractoriness in dogs. PACE 1999; 22:291-301.

21. Donaldson RM, Taggart P, Nashat F, et al. Study of the electrophysiological effects of early or subendocardial ischaemia with intracavitary electrodes in the dog. Clinical Science 1983; 65:579-588.

22. John RM, Taggart P1, Sutton PM, et al. The interrelation between monophasic action-potential duration, cycle length and ischaemia in the human left ventricle. Eur Hearl J 1992; 13:310-315.

23. Taggart P, Sutton PMI, Swanton RH, et al. Simultaneous endocardial and epicardial monophasic action-potential recordings during brief periods of coronary artery ligation in the dog: influence of adrenaline, bela blockade and alpha blockade. Cardiovasc Res 1988; 22:900-909.

24. Lukas A, Antzelevitch C. Dilferences in the electtophysiological response of canine ventricular epicardiun and endocardium to ischemia. Circulation 1993; 88:2903-2915.

25. Yao $Z$, Cavero I, Gross GJ. Activation of cardiac $\mathrm{K}_{\mathrm{ATP}}$ channels: an endogenous protective mechanism during repetitive ischenia. Am.J Physiol 1993; 264:H495-H504.

26. Kanda A, Watanabe 1, Williams ML, et al. Unanticipated lessening of the rise in extracellular potassium during ischemia by pinacidil. Circulation 1997; 95:1937-1944.

27. Watanabe I, Kanda A, Engle CL, et al. Comparison of the effects of regional ischemia and hyperkalemia on the membrane action potentials of the in situ pig heart. J Cardiovasc Electrophysiol 1.997; 8:1229-1236.

28. Vos MA, de Groot SHM, Verduyn SC, et al. Enhanced susceptibility for acquired torsade de pointes arrhythmias in the dog with chronic, complete AV block is related to cardiac hypertrophy and electrical remodeling. Circulation 1998; 98:1125-1135.

29. Miyazaki T, Moritani K, Miyoshi S, et al. Nicorandil augments regional ischemia-induced monophasic action-potential shortening and potassium accumulation without serious proarrhythmia. J Cardiovasc Pharmacol 1995; 26:949-956.

30. Miyazaki T, Zipes DP. Protection against autonomic denervation following acute myocardial infarction by preconditioning ischemia. Cire Res 1989; 64:437-448.

31. Volders $P G A$, Sipido $K R$, Carmeliet $E$, et al. Repolarizing $\mathrm{K}^{+}$currents $\mathrm{I}_{\mathrm{TO} 1}$ and $\mathrm{l}_{\mathrm{ks}}$ are larger in right than lefi canine ventricular midmyocardium. Circulation 1999; 99:206-210.

32. Lee SD, Dorian P, Geist M, et al. Validation of a noninvasive measure of local myocardial repotarization in a conscious human model: adaptation of repolarization to changes in rate. J Cardiovasc Electrophysiol 1999; 10:1171-1179.

33. Denardo S], Stevenson WG. Is the adaptation of right ventricular refractoriness to an abrupt increase in heart rate impaired in chronic heart failure? Am Hearl J 1989; 117:585-589.

34. Leerssen HM, Vos MA, den Dulk K, et al. Inter- and intraindividual vartations in shortening of ventricular ellective refractory period after an abrupt decrease in pacing cycle length. PACE 1994: 17(Pt. II):2079-2083. 
[110] 


\section{Ischemia, Heart Rate, and Ventricular Repolarization}

Editorial by Michiel J. Janse.

Cardiovascular Research, Academic Medical Center, Amsterdam, The Netherlands

Published in J Cardiovasc Electrophysiol 2000; 11:430-431. 
George Ralph Mines was probably the first to note that, after an abrupt change in heart rate, several beats were needed before the ECG attained a constant form. He considered this an interesting example of how "the previous history of the tissue may influence its immediate behaviour."' Later studies confirmed that the heart has a long "memory," that changes brought about by transient changes in rate or rhythm persist for many beats, and that, on a sudden change in heart rate, several hundred beats at the new rate are needed for the refractory period to reach a steady-state. 2.3 Although in many studies the changes in action-potential configuration during acute ischemia have been described (for references see Wit and Janse ${ }^{4}$ ), studies on the dynamic behavior of action-potential duration and refractory period after sudden changes in heart rate in acutely ischemic myocardium are rare; therefore, the article by Leerssen et al. ${ }^{5}$ (Chapter 7 ) is a welcome addition.

In an earlier study, the authors used action-potential duration, ventricular effective refractory period, and stimulus-to-T interval (STI) to describe the repolarization dynamics after a change in pacing rate. ${ }^{6}$ Although it is generally accepted that these parameters are closely related in normal myocardium, the association between action-potential duration and refractory period in acutely ischemic myocardium becomes lost because of the development of postrepolarization refractoriness. ${ }^{7-11}$ This is due to the fact that the recovery from inactivation of both the fast sodium current and the slow calcium current is markedly delayed in partially depolarized cells, an effect that is potentiated by hypoxia. ${ }^{10,11}$ In the human heart, during the first 3 minutes of global ischemia, action potentials shorten while refractory periods prolong. ${ }^{12}$ It follows that, in acutely ischemic myocardium, no conclusions can be drawn about possible arrhythmogenic consequences of inhomogeneities in action-potential duration without taking into account the inhomogeneities in excitability. The difficulties in determining dispersion in refractoriness in ischemic tissue are related to the fact that threshold requirements for stimulating ischemic myocardium rapidly increase and that, by using strong stimuli to determine local refractory periods, one may be misled, because tissue at a considerable distance from the stimulating electrode that has lower threshold requirements may be excited instead of the tissue under the electrode. $^{13}$ The authors are clearly aware of this and have chosen to use only the duration of monophasic action potential. Because the authors used only two catheters to record monophasic action potentials, one in the apex of the right ventricle and the other in the perfusion area of the left anterior descending coronary artery, which was occluded to produce ischemia, no conclusions could be made about inhomogeneities within the ischemic zone after an abrupt decrease in cycle length. To me, the most important result is the finding that the dynamics of action-potential shortening is the same in ischemic and nonischemic myocardium (see Chapter 7, Figure 7.3). This once more shows that ischemia is not the same as hypoxia, because, in hypoxic myocardium, which in itself leads to marked shortening of action potential, an abrupt decrease in cycle length only results in a minimal extra shortening on the order of $6 \mathrm{msec}^{14}$

I am surprised that no mention is made of alternation in action-potential 
duration after a sudden decrease in pacing cycle. This is a property of normal myocardium. ${ }^{2,14}$ In 1962, Noble ${ }^{15}$ had already developed a computer model for Purkinje fibers. His simulated action potentials showed alternans on a sudden change in rate. In his model, $I_{K}$ conductance $\left(g_{k}\right)$ rises slowly during the action potential to a certain value at which repolarization is initiated. After the end of the action potential, $g_{K}$ decrease slowly. The first action potential of a new, faster rate will be shorter because $g_{K}$ is still high when this action potential starts, due to the short preceding diastole, and so less time is needed to reach the value of $g_{k}$ required to initiate repolarization. The diastole preceding the second action potential of the new rate is longer than the diastole preceding the first one, Consequently, $\mathrm{g}_{k}$ will be lower at the start of the second action potential; therefore, it will take more time for $g_{K}$ to reach the level at which repolarization starts and the second action potential will be longer than the first. Alternation is even more pronounced in ischemic myocardium, where it often occurs at rates similar to those of the sinus rate. Most likely this is due to activation of the calcium current only during every second beat. A small, short action potential then would be the result of activation of the depressed, sodium current only; the large, long action potential would be due to activation of both sodium and calcium inward currents. When, at slow driving rates, action potentials of ischemic cells do not show alternation in action-potential amplitude and duration, an increase in pacing rate immediately results in alternation. Both in experimental animals and in patients dying suddenly during ambulatory monitoring, such alternans often precedes the onset of ventricular arrhythmias (for references see Wit and Janse ${ }^{4}$ ). I have no explanation for the absence of alternans in this study, but it may be related to the "off-line analysis using 'ECG-view' software" to determine the changes in action-potential duration.

In conclusion, I think this is an important study, and it is rather surprising that, until now, so little was known about the kinetics of action-potential shortening after changes in heart rate in ischemic myocardium. It would be most important to determine in future studies whether abrupt increases in heart rate result in inhomogeneities in repolarization in ischemic myocardium, as, in the setting of acute ischemia, lethal arrhythmias can be elicited easily by a sudden shortening in the pacing cycle.

\section{References}

1. Mines GR. On dynamic equilibrium in the heart. J Physiol 1913; 46:349-383.

2. Janse MJ, van der Steen ABM, van Dam RT, et al. Refractory period of the dog's ventricular myocardium following sudden changes in frequency. Circ Res 1969; 24:251-262.

3. Han J, Moe GK. Cumulative effects of cycle length on refractory periods in cardiac tissues. Am J Physiol 1969; 217:106-109.

4. Wil AL, Janse MJ. The ventricular arrhythmias of ischemia and infarction: electrophysiological mechanisms. Mount Kisco, NY, Futura Publishing Company, Inc., 1993, pp. 184-203. 
5. Leerssen HM, Vos MA, Houben R, et al. High uniformity of left and right ventricular repolarization dynamics induced by an abrupt decrease in pacing cycle length in a dog is nol affected by left-ventricular ischemia. J Cardiovasc Electrophysiol 2000: 11:421-429.

6. Leerssen HM, Vos MA, den Dulk K, et al. Steady-state and dynamic behavior of ventricular repolarization and refractoriness in the dog. PACE 1998; 21:1766-1777.

7. El-Sheri $\mathrm{N}$, Scherlag BJ, Lazzara R. Electrode catheter recordings during malignant ventricular arrhythmia following experimental acute myocardial ischemia. Evidence for re-entry due to conduction delay and block in ischemic myocardium. Girculation 1975; 51:1003-1014.

8. Downar E, Janse MJ, Durrer D. The effect of acute coronary artery occlusion on subepicardial transmembrane potentials in the intact porcine heart. Circulation 1977; 56:217-224.

9. Lazzara R, El-Sherif N, Hope RR, et al. Ventricular arrhythmias and electrophysiological consequences of myocardial ischemia and infarction. Circ Res 1978; 42:740-749.

[114] 10. Gettes LN, Reuter H. Slow recovery from inactivation of inward currents in mammalian myocardial fibres. J Physiol 1974; 240:703-724

11. Kodama I, Wilde AA, Jande MJ, et al. Combined effects of hypoxia, hyperkalemia and acidosis on membrane action potential and excitability of guinea-pig ventricular muscle. J Mol Cell Cardiol 1984; 16:247-259.

12. Sutton $P$, Taggart $P$, Opthof $T$, et al. Time course and dissociation of action-potential duration and refractoriness during carly ischemia in patients with coronary heart disease. (abstract) Circulation 1997; 96(Suppl): l-497.

13. Janse MJ, Capucci A, Coronel R, et al. Variability of recovery of excitability in the normal canine and the ischamic porcine heart. Eur Heart J 1985; 6(Suppl D):41-52.

14. Rosenbaum DS, Kaplan DT, Kanai $A$, et al. Repolarization inhomogeneities in ventricular myocardium change dynamically with abrupt cycle-length shortening. Circulation 1991; 84:1333-1345.

15. Noble D. A modification of the Hodgkin-Huxley equations applicable to Purkinje fibre action and pacemaker potential. J Physiol 1962; 160:317-336. 


\section{Chapter 8}

\section{Clinical Application of the Stimulus-T Interval for Antitachycardia Pacing}

Hendrik M. Leerssen, Joep L.R.M. Smeets, Marc A. Vos, Volkert A. Zeylemaker, * Karel den Dulk, and Hein J.J. Wellens.

Department of Cardiology, Cardiovascular Research Institute Maastricht, Maastricht University, The Netherlands, and *Medtronic Bakken Research Center, Maastricht, The Netherlands.

Manuscript submitted for publication. 


\section{Abstract}

Introduction: Success of adaptive antitachycardia pacing (ATP) modes in implantable cardioverter defibrillators is rate-dependent because the tachycardia cycle length (TCL) determines the timing of premature stimuli. In an ideal ATP mode that timing should be based on the duration of refractoriness.

Methods and Results: In 12 patients with atrioventricular nodal reentrant tachycardias (AVNRTs) (9F, 3M, $42 \pm 17$ years) and 10 patients with ventricular tachycardias (VTs) (1F, 9M, 64 7 years) safety and feasibility of a new and automatic ATP mode were tested. In this mode of pacing coupling intervals of premature stimuli are determined by on-line stimulus-T interval (STI) information. Since STI is closely related to the duration of refractoriness this mode will adapt to the duration of refractoriness at the moment and site of stimulation. The ATP burst starts with a stimulus with a pre-programmed coupling interval. This stimulus will evoke an endocardial T-wave and at the moment of T-wave sensing the next stimulus will be delivered. 60 Episodes of AVNRT were induced of which 50 were terminated and 25 episodes of 15 different VTs could be induced of which 17 were terminated. The mean coupling percentages for the first four stimuli were 83, 68 , 64 , and $61 \%$. The relation was inverse rate-dependent with shorter coupling intervals for longer TCLs. This means that coupling percentages adapt to the actual duration of refractoriness.

Conclusion: (1)The coupling intervals of premature stimuli in a STI-based ATP burst show an automatic decrement based on the actual duration of refractoriness. (2) STI-based ATP is feasible and usually safe. (3) Success should be evaluated in comparison to current ATP modes. 


\section{Introduction}

Since the introduction of the implantable cardioverter defibrillator (ICD) ${ }^{1}$ the implantable shock-box has developed into a multi programmable tiered therapy device with bradycardia pacing, antitachycardia pacing (ATP), cardioversion and defibrillation therapy. In these devices monomorphic ventricular tachycardia (VT) should preferably be treated by ATP. ${ }^{2}$ Studies report a success rate of ATP between $70-95 \%$, with acceleration rates between $2-7 \% .^{3-5}$ It is even suggested that this therapy should be routinely used not only in patients in whom monomorphic VT was induced during electrophysiologic testing but also in patients who are non-inducible, 6,7

However, success of ATP is cycle-length dependent even for adaptive ATP modes in which coupling intervals are a programmable percentage of the detected tachycardia cycle length (TCL) 8.9 The highest success of these ATP modes is reached at TCLs around $350 \mathrm{msec}^{3-6,8,10,11}$ with a decline in success for both shorter and longer TCLs. ${ }^{3,5}$ This cycle-length dependent success of ATP could be related to the fact that ATP modes adapt to the detected TCL but not to the actual duration of refractoriness. A premature stimulus with a coupling interval of $75 \%$ could be appropriate for a slow tachycardia whereas the same percentage could be too short for a fast tachycardia. Furthermore, the relation between refractoriness and cycle length is not fixed and can be influenced by external factors such as antiarrhythmic drugs, posture or autonomic tone. As a consequence similar tachycardia rates do not necessarily have similar durations of refractoriness and for the same tachycardia refractory periods can be inconsistent between different days. ${ }^{10,12,13}$ Therefore coupling intervals of premature stimuli of an ideal ATP mode should not be a percentage of the TCL, but should be adapted to the actual duration of refractoriness on a beat-to-beat basis. ${ }^{1+}$

In earlier work we described the close relation between the ventricular effective refractory period, action-potential duration and the stimulus-T interval (STI), ${ }^{15}$ not only during different steady-state conditions but also in the dynamic situation after various abrupt pacing cycle length decreases..$^{10}$

Unlike action-potential duration and ventricular effective refractory period, STI can be made on-line available in implantable pacing systems using normal chronically stable pacemaker leads without the use of special pacing protocols. ${ }^{15}$ Therefore, beat-to-beat information of the duration of repolarization and refractoriness in the individual patient can be made available. Using this opportunity we developed an ATP mode in which coupling intervals of premature stimuli are automatically determined based on on-line STI information. ${ }^{14,17}$

The purpose of these experiments was to test the feasibility and safety and to study the characteristics of STI-based ATP in patients. It was not the intention of this study to test the efficacy of STI-based ATP in comparison to other ATP modes. 


\section{Methods}

\section{Patients}

The experiments were performed during electrophysiological evaluation of patients with atrioventricular nodal reentrant tachycardias (AVNRTs) and patients with VTs in the setting of an old myocardial infarction. In patients with AVNRTs all antiarrhythmic medication was stopped at least 5 half lives before the procedure. Patients were included when the tachycardia was hemodynamically well tolerated with reproducible induction and termination using a standard pacing protocol.

Standard Induction and Termination Protocol for AVNRT and VT

The protocol used has been described before. ${ }^{18-20}$ Briefly, for tachycardia induction a 10 stimulus train with a 600,500 or $430 \mathrm{msec}$ cycle length was followed by 1, 2 or 3 premature stimuli. The coupling interval of the first premature stimulus was shortened by $10 \mathrm{msec}$ each time the pacing train was repeated until this stimulus failed to capture because the coupling interval was shorter than the refractory period. When this occurred the coupling interval of the first premature stimulus was programmed $20 \mathrm{msec}$ longer than the refractory period and the second premature stimulus was added. The same scanning procedure was repeated for the second and if necessary the third premature stimulus.

If the tachycardia was hemodynamically tolerated ventricular premature beats were used to terminate the tachycardia.

\section{Experimental Setup}

The pace-sense catheter used in this study was a standard quadripolar EP catheter (BARD electrophysiology, Billerica, MA, USA) with polished platinized electrodes $2 \mathrm{~mm}$ wide with $1 \mathrm{~cm}$ inter-electrode distance. Sensing was performed between the tip and the most proximal ring. This results in an electrode distance for pacing and sensing of $3 \mathrm{~cm}$. In a pilot study we found that the amplitude of the evoked T-wave of this sense configuration was very similar to a unipolar configuration. Using a bipolar sense configuration the amplitude of the evoked T-wave was too low.

The pace-sense catheter was positioned in the apex of the right ventricle, the conventional location of chronic electrodes. This position might not be optimal for termination of AVNRTs. Pacing and sensing was performed with the evoked response stimulator (Vitatron Medical B.V., Dieren, The Netherlands). This stimulator allows polarization free recording of the endocardially-evoked response ${ }^{15}$ and can be triggered by an external source. Patients were paced at twice diastolic threshold.

The evoked response stimulator generates a stimulation marker (indicating the moment of stimulation), an R-wave marker (indicating the moment of R-wave sensing from the endocardially-evoked R-wave) and a $\mathrm{T}$-wave marker (indicating 
the moment of $\mathrm{T}$-wave sensing from the endocardially-evoked $\mathrm{T}$-wave) (Figure $8.3,8.5$, and 8.7). Sensitivity for the R-wave was programmed to half the R-wave. amplitude and T-wave sensitivity was programmed to one third of the I-wave amplitude.

In this custom-build stimulator T-wave detection is realized by performing an adjustable detection on the inverted derivative of the intracardiac signal. The T-wave detection is enabled in an adjustable window to focus on the falling edge of the evoked T-wave. This window is reset after every stimulus.

A data storage and monitoring system based on a personal computer (Department of Medical Technology, Maastricht University, Maastricht, The Netherlands) allows simultaneous storage and monitoring of the surface ECG, stimulus marker, T-wave marker, R-wave marker, and the endocardially-evoked response.

The stimulus-marker, $\mathrm{T}$-wave marker, and $\mathrm{R}$-wave marker are fed into the evoked response feedback system. This personal computer generates the trigger signal for the stimulator. This trigger is defined by the program (Bakken Research Center Medtronic, Maastricht, The Netherlands) which is build as a statemachine with the following main states: VT induction, VT termination, and VVI pacing.

\section{STI-based ATP Protocol}

Figure 8.1 is a schematic representation of this protocol. In this example the VI cycle length is RR msec, the coupling interval of the first stimulus of the ATP burst is RS1 msec and the total amount of premature stimuli in the ATP burst is 5 .

Detection: After a programmable amount (4 - 12) of intervals with a cycle length (RR msec) shorter than the tachycardia detection interval the rhythm will be classified as tachycardia and ATP will start.

Re-detection: If, after ATP, the tachycardia continuous the tachycardia will be redetected after a programmable amount of intervals (4 - 12).

Amount of bursts: The amount of bursts delivered during one ATP therapy is programmable $(3-5)$. ATP will stop if either RR intervals become longer than the tachycardia detection interval or after the last ATP burst has been delivered.

Coupling intervals in burst: The coupling interval of the first stimulus in the burst (RS1, Figure 8.1) is a programmable percentage ( $75-90 \%$ ) of the mean of the 4 preceding RR intervals. This stimulus will evoke an endocardial response. The moment of T-wave sensing will mark the end of the refractory period and the end of the first STI (STI1, Figure 8.1). The second stimulus will be delivered at the moment of T-wave sense of STI1. Again this stimulus will evoke an endocardial response and the end of STI2 will be marked by the moment of T-wave sense and the stimulus to start STI3. This process continues for a programmable amount of stimuli $(2-9)$.

Increasing number of stimuli: The number of stimuli in subsequent bursts of an ATP therapy can be increased automatically. The increase per burst is 1 stimulus and the maximum amount of stimuli in a burst is 9 . 
Shortening of coupling interval of the last stimulus in a burst: The coupling interval of the last stimulus in the burst can be reduced by a programmable amount of $\mathrm{msec}(10-25 \mathrm{msec})$ compared to the preceding STI. E.g. Figure 8.1, if the burst includes 5 stimuli (S1-S5) the coupling interval of the last stimulus (S4S5) can be programmed $10-25$ msec shorter than the last measured STI (STI3).

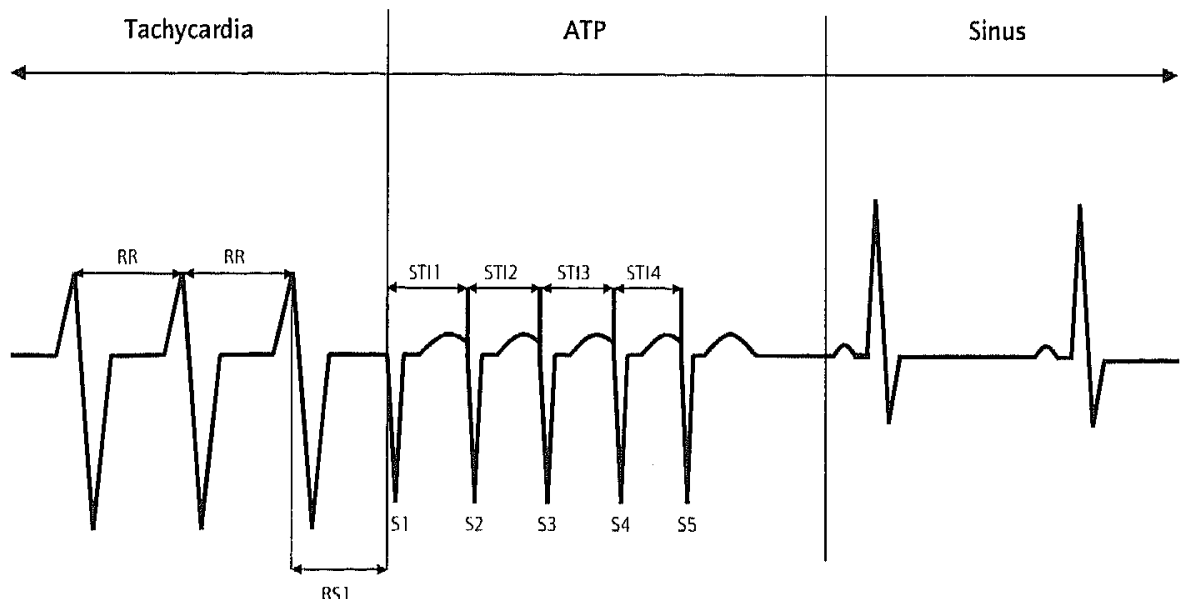

Figure 8.1 - Schematic representation of the automatic antitachycardia pacing (ATP) protocol based on beat-to-beat stimulus-T interval (STI) information.

At a given tachycardia cycle length (RR msec) a premature stimulus (S1) is delivered with a pre-programmed coupling interval (RS1 msec). This premature stimulus will generate an endocardially-evoked response. The first stimulus-T interval (STII) starts with SI and ends at the moment of T-wave sensing in the downgoing slope of the evoked T-wave. The second premature stimulus (\$2) is triggered by the moment of T-wave sensing of the preceding evoked T-wave. T-wave sensing after 52 will mark STI2 and trigger S3. This process continues for the programmed number of stimuli. See Methods, paragraph "STI-based ATP protocol", for details. Abbreviations: STI = stimulus-T interval; ATP $=$ antitachycardia pacing.

\section{Data Analysis and Statistics}

The tracings were analyzed using "ECG-view" software (developed in collaboration with the Department of Medical Technology, Maastricht University, Maastricht, The Netherlands). The settings of this software allowed interval measurements with $5 \mathrm{msec}$ accuracy.

Tachycardias were categorized as non-sustained if the duration was shorter than $30 \mathrm{sec}$.

Coupling intervals of premature stimuli in the STI-based ATP burst for TCLs $\geq 413$ and TCLs $<413$ msec were compared using ANOVA.

The software used for the statistical tests was Primer of Biostatistics (McGraw-Hill Inc., New York, NY, USA), P values $<0.05$ were considered significant. 


\section{Results}

Patients and Tachycardias

The clinical characteristics of the patients with AVNRTs and VTs together with the most important data of the tachycardias are shown in Table 8.1 and 8.2.

\section{STI-based ATP for AVNRT}

In 12 patients 60 episodes of AVNRTs were induced with a mean cycle length of $346 \pm 78 \mathrm{msec}$ (Table 8.1 ). In 10 patients 50 episodes could be terminated. Figure 8.2 is a graphic representation of the coupling intervals of one successful STI-based ATP attempt in each of these 10 patients. Figure 8.3 shows an example of ATP in patient 10.

The 50 successful STI-based terminations were preceded by 39 attempts that did not terminate the AVNRT. In 13 episodes success could be achieved by adding 1 extra stimulus. In 5 episodes repetition of the same number of stimuli with the same coupling intervals terminated the tachycardia. In 20 episodes either T-wave $(n=19)$ or R-wave $(n=1)$ sensing problems were the cause of failure. In the Discussions section "Technical aspects of T-wave sensing" the background and a possible solution of this problem will be discussed. In 1 episode the last stimulus was within the refractory period and did not capture because the additional decrement of the coupling interval for the last stimulus was programmed to high.

In 2 patients STI-based ATP was not successful (Table 8.1). In patient 5 four episodes of tachycardia did not respond to STI-based ATP with 4,5 and 7 stimuli whereas burst pacing with 3,4 and 25 beats did stop the tachycardias. In patient 12, two AVNRTs could not be terminated with STl-based ATP from the right ventricular apex. These AVNRTs could be terminated from the coronary sinus.

In 3 patients STI-based ATP was pro-arrhythmic (Table 8.1). STI-based ATP terminated 4 AVNRTs and induced 1 VT in patient 1. The VT was terminated immediately thereafter with STI-based ATP. In patient 9, two AVNRTs were terminated and A-fib was induced once. In patient 10, thirteen AVNRTs were terminated. In 2 cases, non-sustained VT preceded termination.

\section{STI-based ATP for VT}

In 10 patients, 25 episodes of sustained, hemodynamically tolerated VT with 15 different morphologies and a mean cycle length of $337 \pm 105 \mathrm{msec}$ were induced (Table 8.2). Seventeen episodes with 10 morphologies were terminated in 8 patients by STI-based ATP. Figure 8.4 is a graphic representation of the coupling intervals in the STI-based ATP bursts during these terminations. Figure 8.5 shows an example of ATP in patient 8.

In 4 of 17 successful terminations the first attempt of STI-based ATP was immediately successful. In 13 episodes the successful attempt was preceded by 


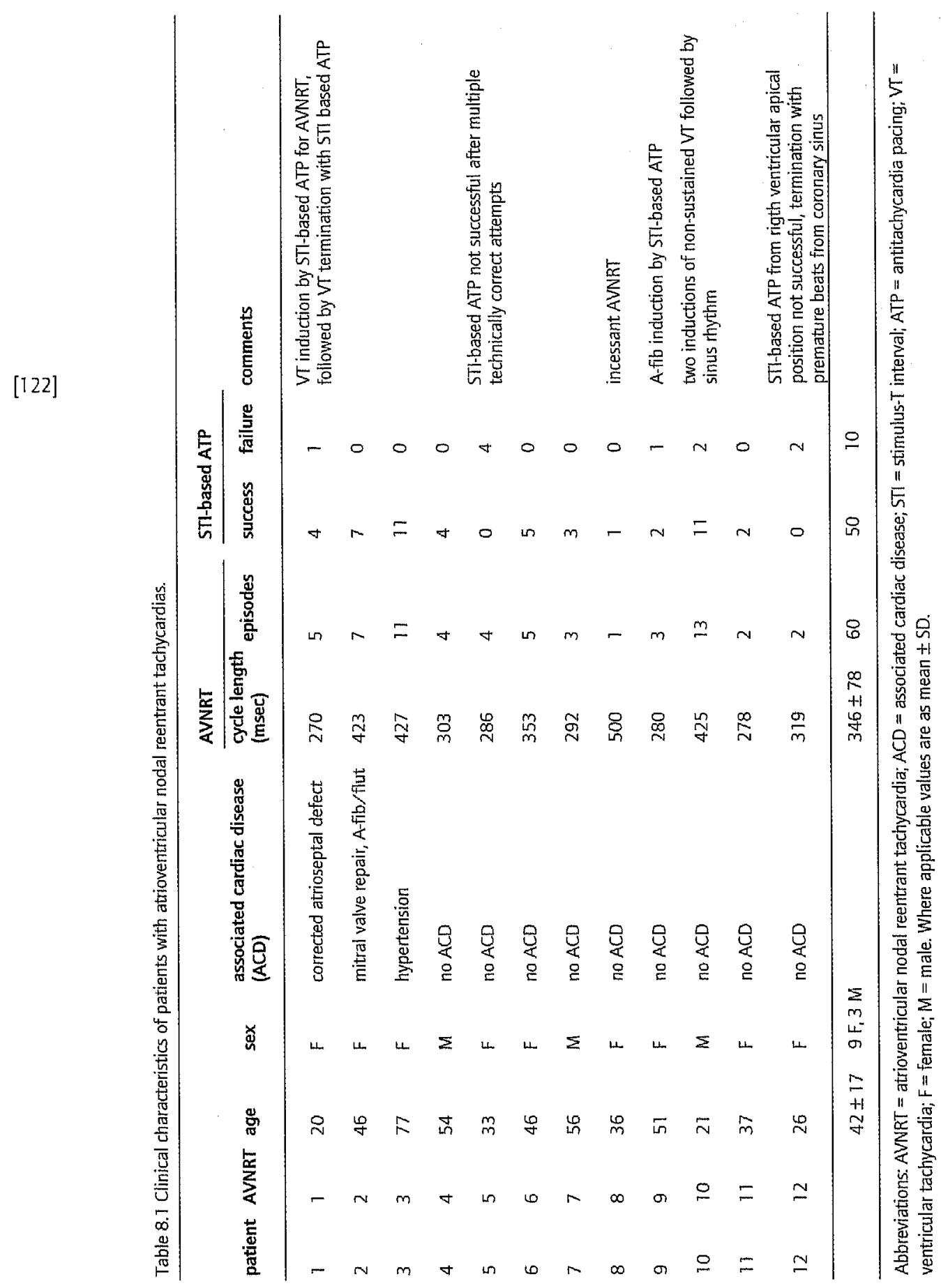




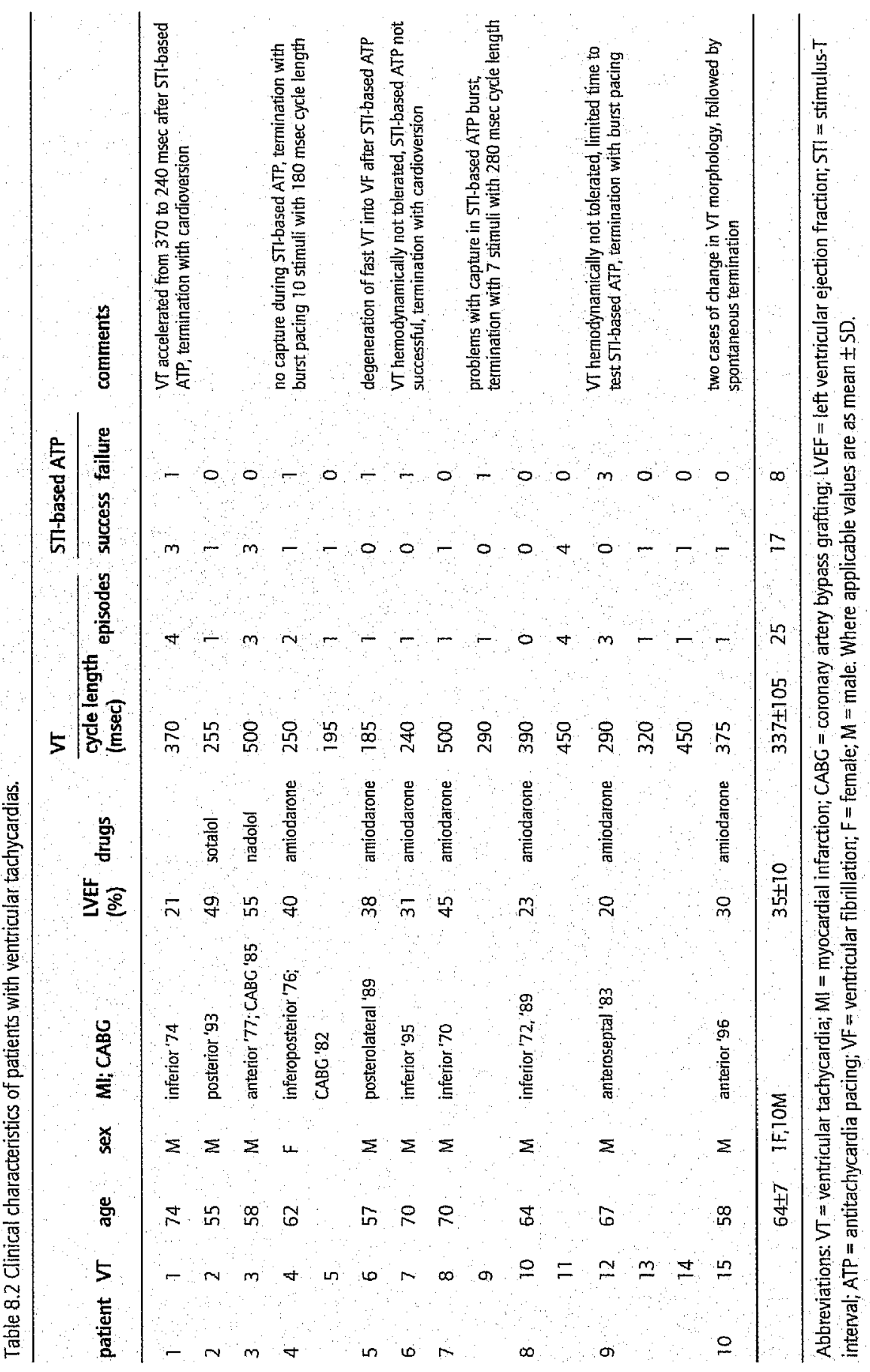




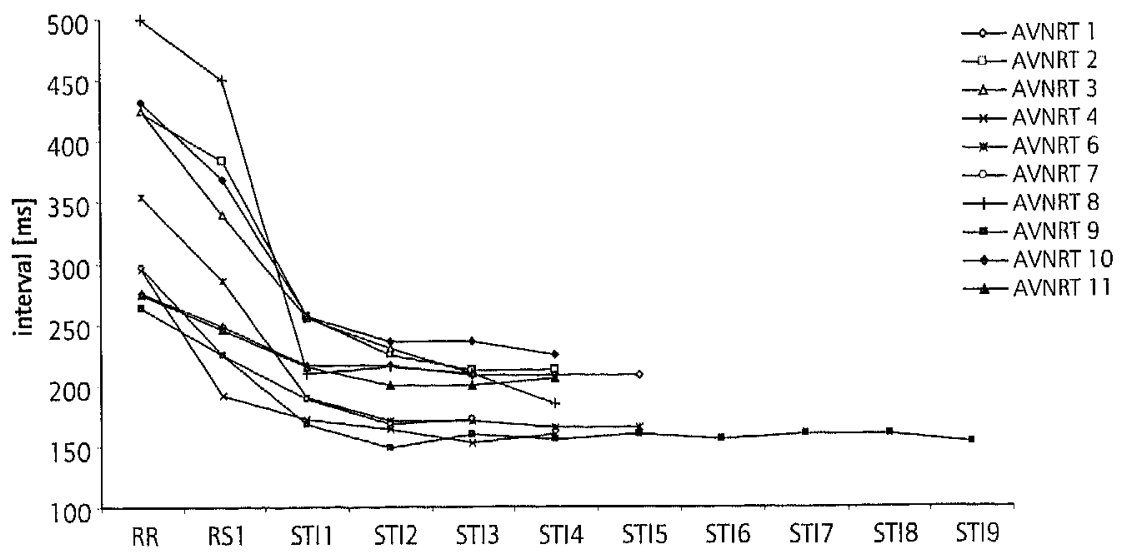

Figure 8.2 - Cycie length of the atrioventricular nodal reentrant tachycardia (AVNRT) and coupling intervals of premature stimuli during successful stimulus-T interval (STI) based antitachycardia pacing (ATP). The index numbers of the AVNRTs correspond with numbers in the column AVNRT of Table 8.1. RR is the cycle length of the AVNRT, RS1 is the pre-programmed coupling interval of the first premature stimulus. STI1, STI2, STI3, ..., STI9 represent the stimulus-T intervals in the burst. See Methods paragraph "STI-based ATP protocol" for details and Figure 8.1. Abbreviations: AVNRT = atrioventricular nodal reentrant tachycardia; $S T I=$ stimulus-T interval; ATP $=$ antitachycardia pacing.

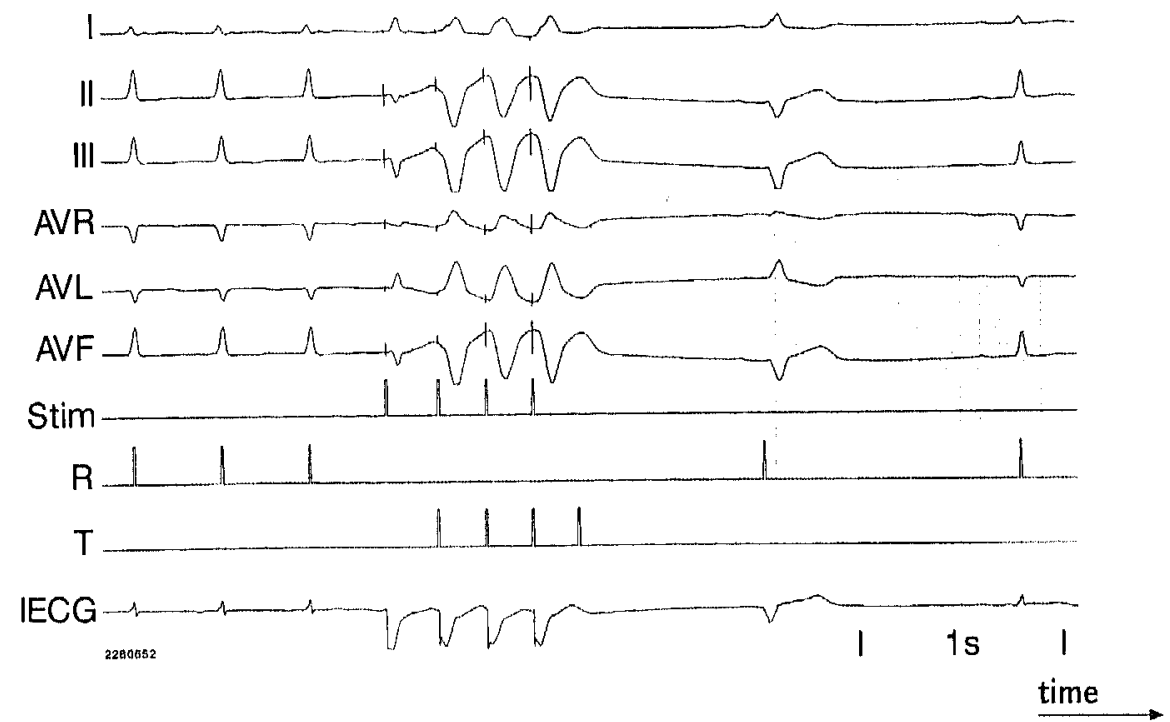

Figure 8.3 - An example of stimulus-T interval (STI) based antitachycardia pacing (ATP) for the atrioventricular nodal reentrant tachycardia (AVNRT) in patient 10 (see also Table 8.1 and Figure 8.2).

ECC leads I, II, III, AVR, AVL and AVF are shown. Tracing "Stim" is the marker tracing indicating pacing. Tracing " $R$ " is the marker tracing indicating R-sense from the intracardiac electrocardiogram. Tracing "T" is the marker tracing indicating T-sense from the evoked T-wave. Tracing "IECC" is the intracardiac electrocardiogram. 
unsuccessful ATP bursts. In 8 of these 13 episodes T-wave $(n=6)$ or R-wave $(\mathrm{n}=2)$ sensing was not correct. In the Discussions section "Technical aspects of T-wave sensing" the background and a possible solution of this problem will be discussed. In the remaining 5 episodes there was no difference between the successful and failing burst in one episode. In one episode the VT morphology changed, with subsequent termination of the new VT morphology with the next burst with the same amount of stimuli. Shortening of the coupling interval of the first stimulus (RS1) made the burst successful in 2 episodes and in 1 episode an increase in the amount of stimuli in the burst from 1 to 3 made the burst successful.

In 8 of 25 episodes STI-based ATP did not terminate the VT. STI-based ATP resulted once in VT acceleration and once in VT degeneration into VF The VT accelerated spontaneously before STI-based ATP could be applied, in four cases. In the remaining 2 episodes, STI-based ATP was not successful because of failed capture.

The Relation Between Tachycardia Cycle Length and the Coupling Percentage of Premature Stimuli in the STI-based ATP Burst

During the experiments 67 tachycardias (50 AVNRTs and $17 \mathrm{VTs}$ ) were successfully terminated with STI-based ATP. A comparison was made between RS1, STII, STI2, STI3, and STI4 for tachycardias with a cycle length shorter than the median TCL of $413 \mathrm{msec}(\mathrm{n}=33)$ and for tachycardias with a cycle length equal to or longer than the median TCL $(n=34)$. This comparison was limited to 4 stimuli (STI4) since only a few bursts included more than 4 stimuli (Figure 8.6). The coupling intervals are expressed as percentage of the TCL.

There was no difference for the pre-programmed coupling interval RS1 of the first premature stimulus ( $83 \pm 8$ versus $81 \pm 9 \%$ ), whereas STI1, STI2, STI3, and STI4 shortened significantly more for the slower tachycardias (STII: $68 \pm 9$ versus $58 \pm 7 \%(\mathrm{P}<0.001) ;$ STI2: $64 \pm 11$ versus $54 \pm 9 \%(\mathrm{P}<0.001) ; \mathrm{STI} 3: 61 \pm$ 11 versus $50 \pm 9 \%(P<0.001)$; STI4: $61 \pm 13$ versus $49 \pm 9 \%(P=0.002))$.

As shown in Figures 2, 4, and 6 the majority of automatic STI shortening takes place before the fourth stimulus, irrespective of the cycle length of the tachycardia.

Proarrhythmia of STI-based ATP and Coupling Percentages of Premature Stimuli

During 60 AVNRT terminations four new arrhythmias occurred. STI-based ATP induced 1 episode of VT (Table 8.1: patient 1). The cycle length of the AVNRT was $270 \mathrm{msec}$ and the shortest coupling interval in the burst was $78 \%$. Atrial fibrillation was induced once (Table 8.1: patient 9) after STI-based ATP for an AVNRT with a cycle length of $280 \mathrm{msec}$. The shortest coupling interval in the burst was $59 \%$. Two episodes of non-sustained VT were induced in one patient (Table 8.1: patient 10) after STI-based ATP for AVNRTs with a cycle length of $425 \mathrm{msec}$. The shortest coupling intervals in the burst were 47 and $43 \%$. During 50 successful terminations coupling percentages as low as $42 \%$ occurred during 


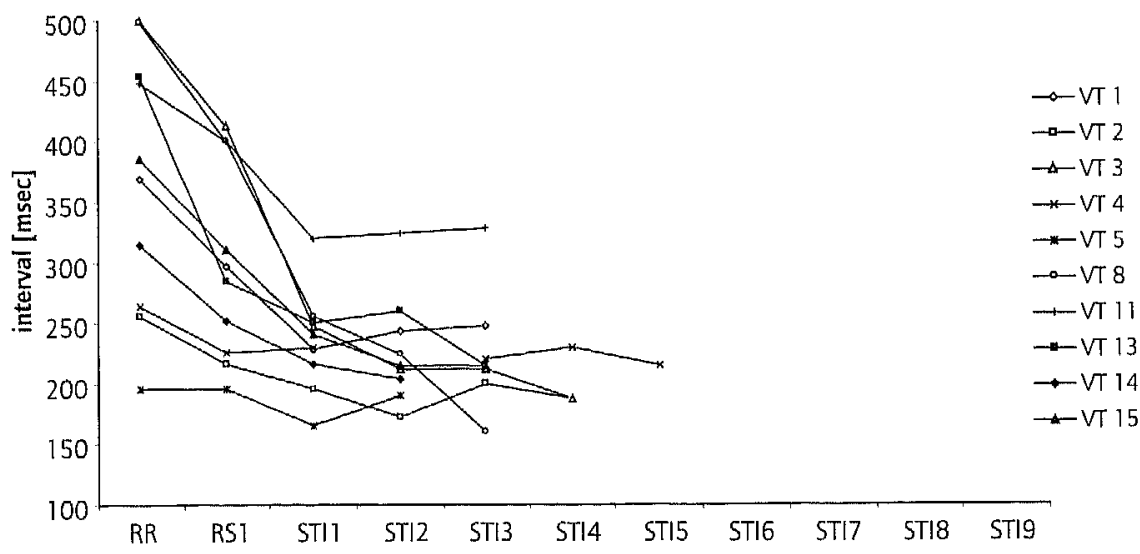

Figure 8.4 - Cycle length of the ventricular tachycardia (VT) and the coupling intervals of premature stimuli during successful stimulus-T interval (STI) based antitachycardia pacing (ATP).

The index numbers of the VTs correspond with the numbers in the column VT of Table 8.2. RR is the cycle length of the VT, RSI is the pre-programmed coupling interval of the first premature stimulus. STII, STI2, STI3, STIL and STI5 represent the stimulus-T intervals in the burst. See Methods paragraph "STI-based ATP protocol" for details and Figure 8.1. Abbreviations: VT = ventricular tachycardia; $S T I=$ stimulus-T interval; ATP = antitachycardia pacing.

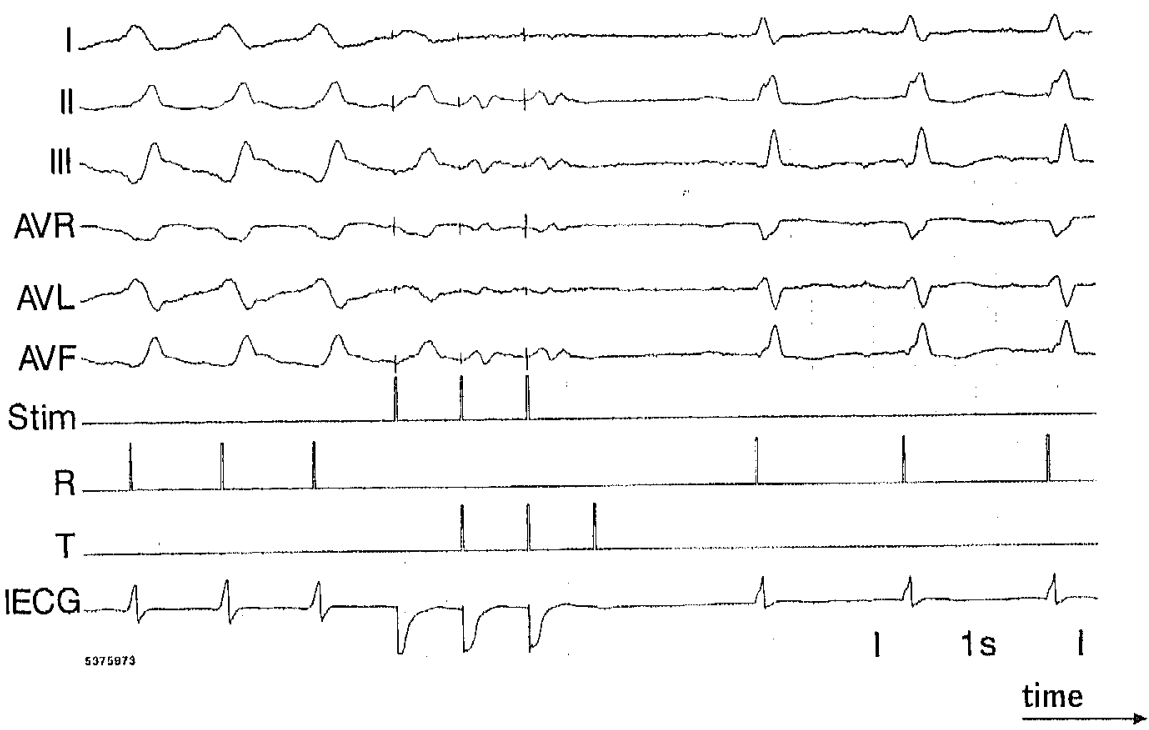

Figure 8.5 - An example of stimulus-T interval (STI) based antitachycardia pacing (ATP) for the ventricular tachycardia (VT) 11 in patient 8 (see also Table 8.2 and Figure 8.4).

ECG leads I, II, III, AVR, AVL and AVF are shown. Tracing "Stim" is the marker tracing indicating pacing. Tracing " $R$ " is the marker tracing indicating R-sense from the intracardiac electrocardiogram. Tracing "T" is the marker tracing indicating T-sense from the evoked T-wave. Tracing "IECC" is the intracardiac electrocardiogram. 


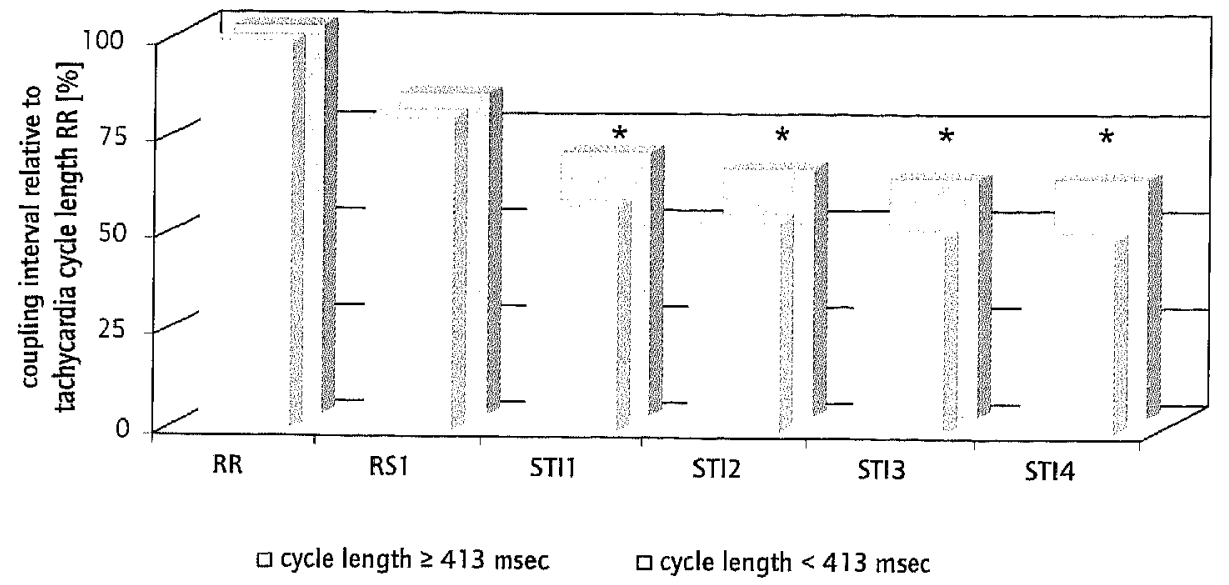

Figure 8.6 - Comparison of the automatic shortening of stimulus-T intervals (STI) during STI-based antitachycardia pacing (ATP) for tachycardias with cycle lengths $\geq 413 \mathrm{msec}$ and tachycardias with cycle lengths $<413$ msec.

RR represents the cycle length of the tachycardia, RSI represents the pre-programmed coupling interval of the first stimulus in the STI-based ATP burst and STI I, STI2, STI3, and STI4 represent the automatically generated subsequent stimulus-T intervals during ATP. The amount of STIs is limited to 4 because only a few bursts included more than 4 stimuli. Significant difference between the cycle lengths is indicated by * (ANOVA, $P<0.005$ ). Abbreviations: STI = stimulus-T interval; ATP = antitachycardia pacing.

STI-based ATP for AVNRTs with cycle lengths between 350 and $500 \mathrm{msec}$ (Table 8.1, Figure 8.2: AVNRT 6, 8 and 10)

During 25 VT terminations, two ATP-related accelerations occurred. The shortest coupling percentages reached during these attempts were $61 \%$ during a VT of $370 \mathrm{msec}$ (Table 8.2: patient 1) and 87\% during a VT of $185 \mathrm{msec}$ (Table 8.2: patient 5). Coupling percentages as low as $42 \%$ for VIs with 500 msec cycle length (Table 8.2, Figure 8.4: VT3 and VT8) did not lead to VT acceleration but successfully terminated VT.

\section{Discussion}

Advantages of STI-based ATP

During STI-based ATP the coupling intervals of premature stimuli in the ATP burst are determined on a beat-to-beat basis by STI information. Since STI is closely related to action-potential duration and the ventricular effective refractory period not only during steady-state circumstances ${ }^{21}$ but also during dynamic circumstances immediately after the onset of tachycardia, 16,22 this ATP mode will adapt automatically to the actual duration of refractoriness in the individual patient on a beat-to-beat basis.

Unlike adaptive ATP modes with fixed coupling percentages in the ATP 
burst, ${ }^{3-5,8,10}$ this automatic STI-based ATP mode adapts the coupling intervals of premature stimuli to the actual duration of refractoriness. In Figure 8.6 we show that this results in significantly lower coupling percentages $(<60 \%)$ for slower tachycardias compared to faster tachycardias $(>60 \%)$. This is caused by the fact that the duration of refractoriness is relatively short compared to the cycle length for slow tachycardias, whereas the duration of refractoriness is relatively long compared to the cycle length for fast tachycardias. Nevertheless, if this relation changes due to external factors such as antiarrhythmic medication or sympathetic stimulation this mode will automatically adapt to these changes. $10,12,13$

The automaticity of STI-based ATP has an additional advantage. As a result of this automaticity the amount of programmable parameters can be considerably lower than for standard adaptive ATP modes. In modern ICDs this could become [128] an important issue.

Proarrhythmia of STI-based ATP and Coupling Percentages of Premature Stimuli

In the literature short coupling percentages during ATP for VT ranging between 65 and $75 \%, 5.10$ are often associated with an increased incidence of tachycardia acceleration. The results of our study show an inverse relationship between TCL and coupling percentages during STI-based ATP, with coupling percentages often lower than $60 \%$ for slow tachycardias (Figure 8.6). The ATP-related VT acceleration and VT degeneration occurred with coupling percentages of 62 and 87 whereas a percentage as low 42 in a VT of 500 msec successfully terminated that VT. Based on these data no comparison between the chance for tachycardia acceleration using standard adaptive ATP modes and STI-based ATP can be made. Nevertheless, these result show that STI-based ATP is successful and safe even with coupling intervals as short as $42 \%$.

Technical Aspects of T-Wave Sensing

To stimulate triggered on a local T-wave, special detection circuitry to process the intra cardiac signal is needed. The detection of T-waves is more complicated than R-wave detection. Normally, R-waves have fast transitions (slewrates) and high amplitudes whereas I-waves are smaller with a frequency content in a lower frequency range. Furthermore the detection of T-waves is influenced by polarization effects of the electrode and by injury potentials just after lead implant. To overcome these difficulties a tri-phasic stimulation technique with active charge compensation has been used. ${ }^{15}$ Using this technique it is necessary to stimulate at regular intervals to keep the baseline on zero. If pacing starts after a longer period without pacing it will take some time to restore the baseline. In our experimental setting the slow polarization compensation effects affected the start of measurements.

Figure 8.7 clearly shows the effect of the initial pacing spikes on the baseline. Due to the offset of the baseline no evoked T-wave can be sensed, since the T-wave sensing window is "open" the evoked R-wave will be falsely sensed as T- 


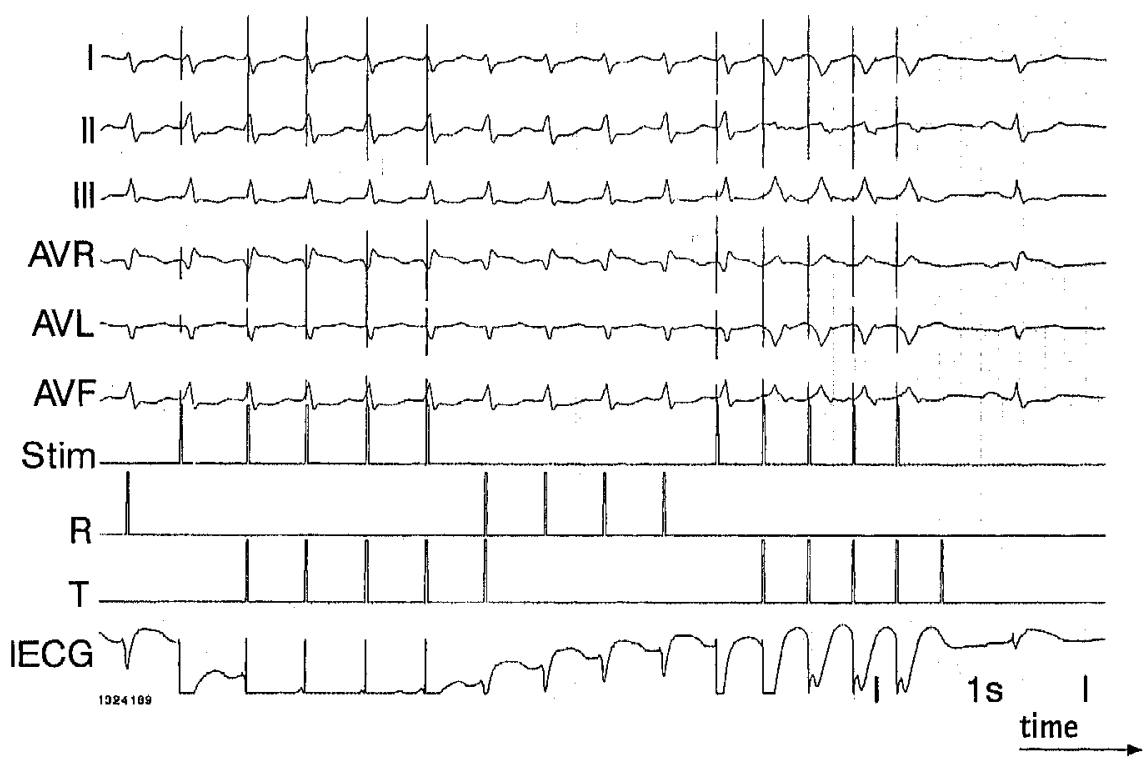

Figure 8.7 - The effect of the initiation of pacing using a low-polarazing tri-phasic pacing pulse. ECG leads I, II, III, AVR, AVL and AVF are shown. Tracing "Stim" is the marker tracing indicating pacing. Tracing " $\mathrm{R}$ " is the marker tracing indicating R-sense from the intracardiac electrocardiogram. Tracing " $\mathrm{T}$ " is the marker tracing indicating T-sense from the evoked T-wave, Tracing "IECC" is the intracardiac electrocardiogram. See Discussion, paragraph "Technical aspects of T-wave sensing."

wave, triggering the next pacing spike. This spike will not capture since it occurs in the QRS complex. The restoration of the baseline is completed before the next ATP burst with adequate T-wave sensing and an effective STI-based ATP burst.

A possible solution for this technical limitation could be R-wave synchronous pacing during the detection phase of the tachycardia before the start of STIbased ATP. Very similar to the example in Figure 8.7 the spikes delivered in the R-wave will help to bring the baseline back to zero without affecting the tachycardia. Other low-polarazing stimulation techniques could also offer a solution of this problem.

Almost $50 \%$ of the 52 failures preceding the 67 successful terminations were caused by problems with T-wave sensing. This high percentage indicates that T-wave sensing is a considerable problem during STI-based ATP. Nevertheless the experiments also show that once the baseline balance is restored, the amplitude of the evoked I-wave is sufficient and the T-wave sensitivity is adjusted accordingly the evoked T-wave can be sensed correctly and STI-based ATP is effective. Considering the experimental setup with an acutely positioned polished platinized pace-sense catheter considerable improvement of the T-wave sensing can be expected by using a chronically stable, low-polarazing pacemaker electrode. 


\section{New Applications for STI-based ATP}

Immediately after the onset of a tachycardia the duration of refractoriness will be longer than when the new steady-state value of refractoriness is reached. Often the number of beats to reach the new steady state ${ }^{16,18,22-24}$ is higher than the number of beats the ICD needs to detect the tachycardia. Therefore shortening of the duration of refractoriness is an ongoing process when ATP starts. Our ATP mode will be able to monitor this shortening behavior and therefore ATP can start immediately after the onset of VT. This option could be used for ATP pacing during fast VTs, which normally require cardioversion. ATP could start immediately after detection of the VT during the charging of the capacitors for high-voltage therapy. If after charging, the tachycardia still exists the charge can be delivered without delay. If ATP stops the tachycardia the painful electrical shock can be avoided. 11

\section{Limitations of the Study}

In this study the safety and feasibility of STI-based ATP from an apical position in the right ventricle of patients with AVNRTs and VTs was confirmed. Although this might not be the ideal position to terminate AVNRTs this stimulation site was chosen because it is the position of conventional chronic electrodes. In our study we did not compare the efficacy of STI-based ATP with conventional ATP modes.

\section{Conclusion}

The results of this study show that during STI-based ATP in patients with AVNRTs and VTs the coupling intervals of premature stimuli automatically adapt to the actual duration of refractoriness. We conclude that in these patients ATP based on real-time STI information is feasible and usually safe.

Acknowledgments: The authors gratefully acknowledge the excellent technical support of Ben Vonk (Vitatron Medical BV, Dieren, The Netherlands).

\section{References}

1. Mirowski M, Reid PR, Mower MM, et al. Termination of malignant ventricular arrhythmias with an implanted automatic defibrillator in human beings. N Engl J Med 1980; 303:322-324.

2. Wellens HJJ. Value and limitations of programmed electrical stimulation of the heart in the study and treatment of tachycardias. Circulation 1978; 57:845-853.

3. Kantoch MJ, Green MS, Tang ASL. Randomized cross-over evaluation of two adaptive pacing algorithms for the termination of ventricular tachycardia. PACE 1993; 16:1664-1672. 
4. Trappe HJ, Klein H, Kielblock B. Role of antitachycardia pacing in patients with third generation cardioverter defibrillators. PACE 1994; 17(Pt. II):506-513.

5. Newman D, Dorian P, Hardy J. Randomized controlled comparison of antitachycardia pacing algorithms for termination of ventricular tachycardia. J Am Coll Cardiol 1993; 21:1413-1418.

6. Rosenqvist M. Pacing techniques to terminate ventricular tachycardia. PACE 1995; 18(Pt. II) $592-598$.

7. Schaumann A, von zur Mühlen F, Herse B, et al. Empirical versus tested antitachycardia pacing in implantable cardioverter delibrillators. A prospective study including 200 patients. Circulation 1998; 97:66-74.

8. De Belder MA, Malik M, Ward DE, et al. Pacing modalities for tachycardia termination. PACE 1990; 13:231-248.

9. den Dulk K, Wellens HJJ. Antitachycardia pacing: clinical considerations. In KA Ellenbogen, GN Kay, BL Wilkoff (eds.). Clinical cardiac pacing, Philadelphia, W. B. Saunders, 1995, pp. 735 743.

10. den Dulk K, Kersschol IE, Brugada P, et al. Is there a universal antitachycardia pacing mode? Am J Cardiol 1986; 57:950-955.

11. Leitch JW, Gillis AM, Wyse DG, et al. Reduction in defibrillator shocks with an implantable device combining antitachycardia pacing and shock therapy. J Am Coll Cardiol 1991; 18:145151.

12. Fisher JD, Kim SG, Matos JA, et al. Comparative effectiveness of pacing techniques for termination of well-tolerated sustained ventricular tachycardia. PACE 1983; 6:915-922.

13. Kappenberger $L$, Sowton $E$. Programmed stimulation for long term treatment and non-invasive investigation of recurrent tachycardia. Lancet 1981; 1:909-914.

14. den Dulk K, Leerssen HM, Vos MA, et al. Applicability of the stimulus-T interval for antitachycarclia pacing. PACE $1991 ; 14$ (Pt. II):1757-1761.

15. Rickards AF, Norman J. Relation between QT interval and heart rate. New design of physiologically adaptive cardiac pacemaker. Br Heart J 1981; 45:56-61.

16. Leerssen HM, Vos MA, den Dulk K, et al. Steady-state and dynamic behavior of ventricular repolarization and refractoriness in the dog. PACE 1998; 21;1766-1777.

17. Begenann $M$, Boute W, Witkampl FH. Evoked endocardial potentials in tachycardia management. (abstract) PACE 1987; 10(Pt. I):608.

18. Janse MJ, van der Stcen ABM, van Dam RT, et al. Refractory period of the dog's ventricular myocardium following sudden changes in frequency. Circ Res 1969; 24:251-262.

19. Ross DL, Farre J, Bär FWHM, el al. Comprehensive clinical electrophysiologic studies in the investigation of documented or suspected tachycardias; time, stalf, problems and costs. Circulation 1980; 61:1010-1016.

20. Brugada P. Wellens HJJ. Standard diagnostic programmed electrical stimulation protocols in patients with paroxysmal recurrent tachycardias. PACE 1984; 7(PL, I1):1121-1128.

21. Manley BS, Chong EMF, Walton $C$, et al. An animal model tor the chronic sudy of ventricular repolarisation and refractory period. Cardiovase Res 1989; 23:16-20

22. Morgan JM, Cunningham AD, Rowland E. Relationship of the eflective refractory period and the monophasic action-potential duration after a step increase in pacing frequency. PACE 1990; 13:1002-1008 
23. Franz MR, Swerdlow CD, Liem LB, et al. Cycle-length dependence of human action-potential duration in-vivo. J Clin Invest 1988; 82:972-979.

24. Kanaan N, Jenkins J, Childs K, et al. Monophasic action-potential duration during programmed electrical stimulation. PACE 1991; 14:1049-1059. 


\section{Chapter 9}

\section{General Discussion}

Table of Contents

Trigger and Validation

Limitations of Current Antitachycardia Pacing Modes

Why is the Success Rate of Antitachycardia Pacing Related to the VT Cycle Length?

Optimal and Reproducible Timing of ATP Stimuli

STI-Based ATP Versus Conventional ATP

Final Remarks and Recommendations

References 


\section{Trigger and Validation}

The work described in this thesis was triggered by Karel den Dulk. ${ }^{1} \mathrm{He}$ showed in the dog with chronic atrioventricular (AV) block ${ }^{2}$ that shortening of the ventricular effective refractory period (VERP) during pacing-induced ventricular tachycardia (VT) can be predicted by stimulus-T interval ${ }^{3}$ (STI) measurements on a beat-to-beat basis. Based on these findings he suggested that this could be implemented in a new and automatic antitachycardia pacing (ATP) mode. In this ATP mode coupling intervals of premature stimuli are determined by on-line STI data. Theoretically this mode is superior to commonly applied ATP modes because it will automatically adapt to patient specific changes in VERP even if these changes are not related to the VT cycle length. This should enable quicker, safer and more reliable termination of VT by ATP.

Following these observations the principle of STI-based ATP was investigated through collaboration between the Department of Cardiology of the University Hospital Maastricht, Vitatron Medical BV (Dieren, The Netherlands) and The Medtronic Bakken Research Center (Maastricht, The Netherlands). Financial support came from the Department of Economic Affairs of The Netherlands.

Initial experiments were performed under normoxic circumstances using a single change in heart rate to simulate the onset of VT. ${ }^{1}$ Additional experiments were carried out in which the influence of multiple cycle-length changes, ${ }^{4}$ antiarrhythmic drugs, ${ }^{4}$ the combination of very high heart rates and antiarrhythmic drugs ${ }^{5}$ and ischemia was tested. ${ }^{6}$ The results of these experiments confirmed a consistent relation between STI, the duration of refractoriness expressed by VERP and the duration of repolarization expressed by the duration of the action potential. This was the background for the development of a new and automatic ATP mode based on on-line STI data, and justified feasibility tests in patients. ${ }^{7}$

Why developing a new ATP mode? The literature raises the impression that there is no need for another new ATP mode, since current modes are extremely successful. ${ }^{8-13}$ However a detailed analysis will learn differently.

\section{Limitations of Current Antitachycardia Pacing Modes}

The literature shows that ATP modes currently provided by implantable cardioverter defibrillators (ICDs) are powerful VT terminators. Success rates of termination range between $70-95 \%$ with acceleration of VT occurring in $2-7 \%, 8-13$ However, the success and acceleration rate of ATP is cycle-length dependent with lower success rates and higher acceleration rates for faster VTs. The optimal range of VT cycle lengths prone to ATP termination ranges between $300-350$ msec, ${ }^{8-10,12,14-16}$ For VTs with cycle lengths below 300 msec overall success rates remain below $60 \% .12,17-19$ 


\section{Why is the Success Rate of Antitachycardia Pacing Related to the VT Cycle Length?}

The aim of ATP is to deliver a critically timed stimulus which will enter the reentry circuit to create refractoriness both in antegrade and retograde direction. 20 Clinical experience shows that the termination zone during which such stimuli can be effective usually begins just after the end of the local VERP. ${ }^{21,22}$ If the site of pacing is close to or inside the reentry circuit there is a chance that the VT can be terminated by a single premature stimulus. ${ }^{23}$ When comparing slow and fast reentrant VTs, fast VTs will arise from smaller anatomical or functional circuits. During fast reentrant VTs, the size of the excitable gap and thus the duration of the termination zone will be reduced, making successful timing more difficult.

Depending on the coupling interval and the site of the premature stimulus the QRS complex of the tachycardia, evoked by this premature stimulus can be delayed or occurring at the tachycardia cycle length. The morphology of the QRS that follows the premature stimulus can be different from or identical to the QRS during tachycardia. If a premature stimulus generates a QRS complex with the same morphology as the VT appearing at the pacing interval the catheter is in the reentry circuit. Such a site is looked for when catheter ablation of the VT is attempted. 24

In clinical practice the site of ATP will almost always be in the apex of the right ventricle, because that is the preferred position of the ICD lead. The reentry circuit however, is often located in the left ventricle. Only during slow VT rates a single premature stimulus from a right ventricular apical position can travel all the distance to the reentry circuit without colliding with a wavefront coming from the VT circuit. During fast VTs the single premature stimulus will not be able to reach the reentry circuit because it will collide with the wavefronts coming from the reentry circuit.

Using ATP modes with multiple paced beats (Table 1.3, chapter 1) this problem can be overcome. The first premature stimulus which will collide with the wavefront coming from the reentry circuit will prevent depolarization at the site of stimulation. The second premature stimulus can be delivered immediately after the end of the local refractory period. Additional premature stimuli can reduce the local refractory period even further, thereby increasing the prematurity and chance that stimuli will reach the site of reentry. ${ }^{20,22,25}$ However during a fast VT, with ATP from a remote site, with depressed conduction in the intervening tissue and a long refractory period at the site of stimulation successful ATP could be impossible.

\section{Optimal and Reproducible Timing of ATP Stimuli}

Based on these theoretical considerations and clinical observations 21,22 increasing prematurity and number of ATP stimuli will increase the chance that a wavefront from a remote stimulation site will reach the reentry circuit. These con- 
siderations resulted in ATP modes which intend to deliver premature stimuli in a termination window which starts immediately after the end of the refractory period. Since the actual duration of refractoriness is unknown coupling intervals are often programmed relative to the VT cycle length. However, for a certain VT cycle length, the inter- and intraindividual variation of VERP duration might be considerable. Using a method which guarantees optimal reproducibility26 we demonstrated in dogs with chronic AV block, stimulated at a rate of 170 beats per minute VERP differences as high as $35 \mathrm{msec}$ (range: $150-185 \mathrm{msec}$ ). Even repeated measurements in the same dog showed differences in VERP up to $20 \mathrm{msec} .{ }^{27}$ This means that previously determined VERP values will not guarantee appropriate timing at a later point in time, even in the same individual. ${ }^{27}$ The only way therefore to obtain reproducible timing of ATP stimuli relative to VERP duration is by

[136] on-line feed back of patient specific information. Since STI-based ATP is build on on-line feed back of patient specific STI data the timing of premature stimuli relative to VERP will be highly reproducible.

\section{STI-Based ATP Versus Conventional ATP}

To illustrate the difference in timing of premature stimuli during ATP, coupling intervals of STI-based ATP and a conventional ATP mode were computed and compared for 3 VT cycle lengths. Coupling intervals of STI-based ATP are based on the results described in chapter 8.7 Ideally STI-based ATP should be compared with a gold standard for ATP. However this gold standard does not exist. We have chosen an adaptive scanning burst (ASB) ATP mode which achieves a success rate of $92 \%$ for 2097 episodes of VT during follow-up. ${ }^{9}$ The hypothetical VTs which will be treated have cycle lengths of 300,350 and $420 \mathrm{msec}$.

The settings of the two ATP modes will be described briefly. Detection of VT starts after 10 intervals with a cycle length shorter than the programmed VT cutoff rate. If ATP is not successful the VT will be redetected after 6 new VT cycles. Because over $96 \%$ of the VTs will be terminated by four ATP treatments the maximum amount of ATP treatments is four. ${ }^{28}$ The number of stimuli in the first burst is 4 and after every unsuccessful attempt 1 stimulus will be added. Based on the results of chapter 8 the coupling intervals of STI-based ATP stimuli for a VT with a cycle length $<413 \mathrm{msec}$ will be $83,68,64$, and $61 \%$ for every additional premature stimulus. For a VT with a cycle length $\geq 413 \mathrm{msec}$ these percentages are significantly lower: $81,58,54,50,49,48$ and $47 \% .^{7}$ The first coupling interval of the conventional type of ASB is programmed at $81 \%$, the coupling intervals of stimuli within the burst are decremented by $8 \mathrm{msec}$ and the coupling intervals of initial stimuli of successive attempts are also decremented by 8 msec.

Figure 9.1 shows the results. The upper panel shows the coupling intervals of both methods for a VT with $300 \mathrm{msec}$ cycle length. Coupling intervals of STIbased ATP reach the shortest value already during the first attempt. The ASB needs 
The goal of current research ongoing at the Department of Cardiology of the University Hospital Maastricht is to show that STI-based ATP continues to be effective when conventional ATP modes no longer are. If the clinical efficacy of STI-based ATP can be demonstrated STI-based ATP could become the best form of antitachycardia pacing.

\section{References}

1. den Dulk K, Leerssen HM, Vos MA, et al. Applicability of the stimulus-T interval for antitachycardia pacing. PACE 1991; 14(Pt. II):1757-1761.

2. Vos MA, de Groot SHM, Verduyn SC, et al. Enhanced susceptibility for acquired torsade de pointes arrhythmias in the dog with chronic, complete AV block is related to cardiac hypertrophy and electrical remodeling. Circulation 1998; 98:1125-1135.

3. Rickards AF, Norman J. Relation between QT interval and heart rate. New design of physiologically adaptive cardiac pacemaker. Br Heart J 1981; 45:56-61.

4. Leerssen HM, Vos MA, den Dulk K, et al. Steady-state and dynamic behavior of ventricular repolarization and refractoriness in the dog: the effect of multiple cycle-length changes and d-sotalol administration. PACE 1998; 21:1766-1777.

5. Leerssen HM, Vos MA, den Dulk $\mathrm{K}$, el al. Rate-dependent elfects of procainamide on the threshold current for pacing in the selting of postrepolarization refiactoriness. PACE 1999; 22:291301.

6. Leerssen HM, Vos MA, Houben R, et al. High unifornity of left and right ventricular repolarization dynamics induced by an abrupt decrease in pacing cycle length in a dog is not alfected by lefl-venuricular ischemia. J Cardiovase Electrophysiol 2000: 11:421-429.

7. Leerssen HM, Smeets JLRM, Vos MA, el al. Application of the stimulus-T interval for antitachycardia pacing. Submiuted.

8. Kantoch M], Green MS, Tang ASL. Randomized cross-over evaluation of two adaptive pacing algorithms for the termination of ventricular tachycardia. PACE 1993; 16:1664-1672.

9. Trappe $\mathrm{HJ}$, Klein $\mathrm{H}$, Kielblock $\mathrm{B}$. Role of antitachycardia pacing in patients with third generation cardioverter delibrillators. PACE 1994; 17:506-513.

10. Newman D, Dorian P, Hardy J. Randomized controlled comparison of antilachycardia pacing algorthms for termination of ventricular tachycardia. J Am Coll Cardiol 1993; 21:1413-1418.

11. Fiek M, Dorwarth U, Müller D, et al, Predictors of efficacy of antitachycardia pacing for treatment of ventricular tachycardia in patients with implantable cardioverter defibrillator (ICD). (abstract) PACE 1999; 22:A144.

12. Marrouche N, Gerritse B, Brachmann $J$. The efficacy of anti-tachycardia pacing therapy in implantable cardioverter defibrillator patients depending on the average cycle length of the ventricular tachycardia. (abstract) PACE 1999; 22:A140.

13. Schaumann A, Poppinga A, Fabian $O$, et al. Ellicacy of antilachycardia pacing in 160 patients with a history of ventricular fibrillation. (abstract) PACE 1999; 22:A146.

14. Rosenqvist M. Pacing techniques to terminate ventricular tachycardia. PACE 1995; 18:592-598.

15. De Belder MA, Malik M, Ward DE, el al. Pacing modalities for tachycardia termination. PACE 1990; 13:231-248. 


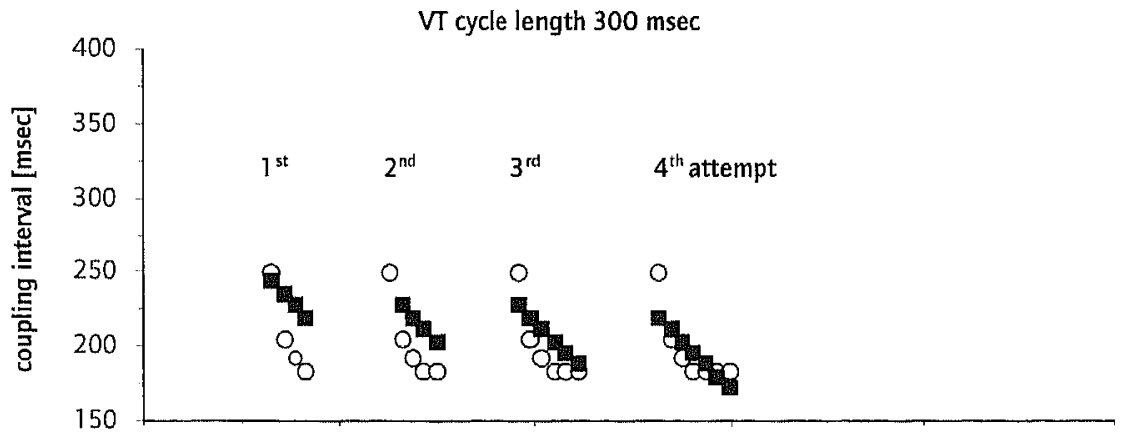

400

VT cycle length 350 msec

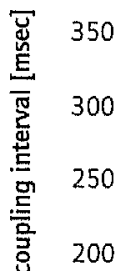
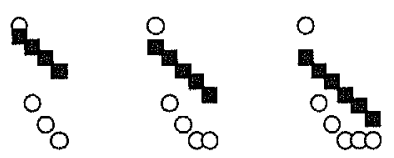

0

150

400

$\mathrm{VT}$ cycle length $420 \mathrm{msec}$

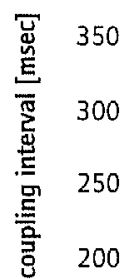
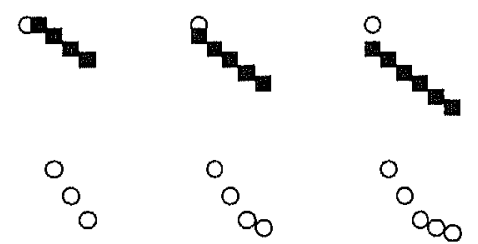

${ }_{\infty}^{\circ}$
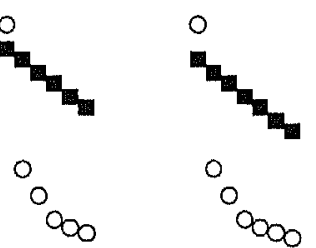

150

0

5

10

15

20

25

time after onset of VT [sec]

O STI based ATP

- Adaptive Scanning Burst (ASB)

Figure 9.1 - Coupling intervals of premature stimuli during ATP.

Abbreviations: $S T I=$ stimulus- $T$ interval; $A T P=$ antitachycardia pacing; $V T=$ ventricular tachycardia. 
3 attempts before coupling intervals decrease to similar values. During the $4^{\text {th }}$ attempt coupling intervals of the ASB decrease to values which could easily fall within the refractory period, with additional shortening for additional attempts.

The middle panel shows the coupling intervals for a VT with a cycle length of $350 \mathrm{msec}$. Under these circumstances the longest and the shortest coupling intervals of both methods generated in 4 attempts are almost identical. However STI-based ATP reaches the shortest coupling interval already during the $1^{\text {st }}$ attempt whereas ASB needs 4 attempts.

The lower panel illustrates the situation for a VT of $420 \mathrm{msec}$. The difference is striking, shortening of coupling intervals during STI-based ATP is much more pronounced than with the ASB method. Using ASB it would take 8 attempts and 40 seconds longer before a premature stimulus with the same coupling interval would be delivered.

The 3 panels clearly show that conventional ATP methods such as ASB, in which coupling intervals of premature stimuli are a relative portion of the VT cycle length, underestimate the duration of the VERP at short cycle lengths and overestimate the VERP at long cycle lengths. During STI-based ATP coupling intervals will automatically adapt to the actual duration of the VERP. This means that coupling percentages for slow VTs will be lower than for fast VTs. ${ }^{7}$ Furthermore a change in VERP not related to VT cycle length, e.g., after a change in antiarrhythmic drugs or different levels of sympathetic stimulation at which the VT occurs, will automatically lead to changes in coupling intervals during STIbased ATP whereas conventional ATP methods will not change their pacing intervals.

\section{Final Remarks and Recommendations}

The initial work by den Dulk et al.' demonstrated a consistent relation between STI and VERP in the dynamic situation after the onset of VT. Based on this relation the idea of ATP using on-line feed back of STI information for timing of premature stimuli was born. Our research confirmed the consistency of the relation between STI and VERP for the variety of circumstance which can be encountered during VT.

The results of this research justified the development of a new STI-based ATP mode. The feasibility and safety of this ATP mode could be demonstrated in patients. It was beyond the scope of this thesis to compare the efficacy of STlbased ATP with conventional ATP modes.

The most unique feature of STI-based ATP is that timing of premature stimuli automatically adapts to the actual duration of refractoriness of the individual patient, which can not be done in conventional ATP modes. Furthermore it should be emphasized that STI-based ATP does not require extensive programming as in current ATP modes. Using this mode, ATP therapy in ICDs could be switched on or off making programming of numerous parameters obsolete. 


\section{Summary}

Chapter 1 provides the framework in which this research has to be placed. A brief overview of the historical landmarks within cardiac electrophysiology is given. One of these landmarks was the introduction of intracardiac electrical recordings and stimulation. Using this technique it could be demonstrated that ventricular tachycardia in the setting of an old myocardial infarction could be initiated and terminated by programmed electrical stimulation. The success of tachycardia termination or antitachycardia pacing was strongly dependent on the site and moment of stimulation in relation to the tachycardia cycle length. If antitachycardia pacing is applied by an implanted pacemaker with a lead in the right ventricle, the chance of successful termination is high when premature stimuli are timed immediately after the refractory period at the site of stimulation. Since the actual duration of refractoriness during antitachycardia pacing is unknown the refractory period has to be estimated.

Almost twenty years ago an implantable pacemaker was developed that could register the electrical response of the heart via the pacemaker electrode immediately after the moment of stimulation. To do so, the pacemaker had to be equipped with a low polarizing electrode and a special stimulus configuration. The stimulus- $T$ interval is a portion of the endocardially evoked signal, that starts at the moment of stimulation and ends in the T-wave. As a consequence, the stimulus-T interval will provide information on the duration of refractoriness. Furthermore it is of importance that the stimulus- $T$ interval can be determined automatically and on a beat-to-beat basis by the implantable pacemaker.

This principle can be used for a new and automatic antitachycardia pacing mode in which coupling intervals of premature stimuli are guided by real time stimulus-T interval information. However, developing such a method for antitachycardia pacing can only be considered if the relation between the stimulus-T interval and the refractory period is consistent for the various circumstances during which antitachycardia pacing will be applied. To study the relation between the stimulus- $T$ interval and the refractory period, a dog model with atrioventricular block has been used. Since the ventricular rate in this model is low, ventricular tachycardia can be simulated by an abrupt and substantial decrease in pacing cycle length. Initial experiments to study the relation between the stimulus-T interval and the refractory period are described in Chapter 2 . The results show that the simulated ventricular tachycardia induces significant stimulus-T interval and refractory-period shortening, with a high uniformity in shortening dynamics. The absolute value of the stimulus- $T$ interval is always longer than the absolute value of the refractory period. Based on these results, the stimulus- $T$ interval could be used to predict the refractory period. 
16. Roy D, Waxman HL, Buxton AE, et al. Termination of ventricular tachycardia: role of tachycardia cycle length. Am J Cardiol 1982; 50:1346-1350.

17. Mason JW, Winkle RA. Electrode-catheter arrhythmia induction in the selection and assessment of antiarrhythmic drug therapy. Circulation 1978; 58:971-985

18. Naccarelli GV, Zipes DP, Rahilly GT, et al. Influence of tachycardia cycle length and antiarrhythmic drugs on pacing termination and acceleration of ventricular tachycardia. Am Heart J 1983; 105:1-5.

19. Fisher JD, Mehra R, Furman S. Termination of ventricular tachycardia with bursts of rapid ventricular pacing. Am J Cardiol 1978; 41:94-102.

20. Wellens HJJ. Value and limitations of programmed electrical stimulation of the heart in the study and treatment of tachycardias. Circulation 1978; 57:845-853.

21. Fisher JD, Ostrow E, Kim SG. Ultrarapid single-capture train stimulation for termination of ventricular tachycardia. Am J Cardiol 1983; 51:1334-1338.

22. Fisher JD, Kim SG, Waspe LE, et al. Mechanisms for the success and failure of pacing for termination of ventricular tachycardia: clinical and hypothetical considerations. PACE 1983; 6:1094-1105

23. Wellens HJJ, Schuilenburg RM, Durrer D. Electrical stimulation of the heart in patients with ventricular tachycardia. Circulation 1972; 46:216-226.

24. Stevenson WG, Khan H, Sager P, et al. Identilication of reentry circuit sites during catheter mapping and radiofrequency ablation of ventricular tachycardia late after myocardial infarction. Circulation 1993; 88:1647-1670.

25. Wellens HJJ, Bär FW, Gorgels AP, et al. Electrical management of arrhythmias with emphasis on the lachycardias. Am J Cardiol 1978; 57:1025-1034.

26. Leerssen HM, Vos MA, den Dulk $\mathrm{K}$, et al. Is the ventricular effective refractory period different when determined by incremental versus decremental scanning?: the effect of pacing cycle length, d-sotalol, and levcromakalim. PACE 1994; 17(Pt. 11):2084-2089.

27. Leerssen HM, Vos MA, den Dulk $K$, et al. Inter- and intraindividual variations in shortening of ventricular effective refractory period after an abrupt decrease in pacing cycle length. PACE 1994; 17(Pt. II):2079-2083.

28. Thamaset $S$, Grossmann $G$, Kochs $M$, et al. Only seven antitachycardia pacing attempts with an implantable cardioverter defibrillator are necessary to terminate a ventricular tachycardia. (abstract) PACE 1999; 22:A139. 
High-precision refractory-period measurements are crucial for this research. In Chapter 3 two protocols that guarantee precise and reproducible values for the refractory period are compared. Although the stimulation protocols of the two methods are different, the results show that the refractory periods determined with these two methods are not different. Using the "decremental-scanning" protocol, multiple and abrupt changes in cycle length will occur during the scanning process. Using the "incremental-scanning" protocol these cycle length changes can be avoided. Therefore incremental scanning might be a better method to measure refractory periods if induction of arrhythmias is to be avoided.

In Chapter 4 the variability of the refractory period for identical circumstances in the same dog at different points in time and the variability for different dogs under identical circumstances was tested. The variability of the refractory [142] periods found in both situations was high. These results demonstrate that refractory periods determined at one point in time have little predictive value for refractory periods at comparable conditions later in time. Once again this emphasizes the need of real-time refractory-period information at the moment of antitachycardia pacing.

In Chapter 5 the behavior of the action-potential duration, stimulus-T interval, and refractory period during a simulated tachycardia is studied. This is done during control conditions and after the administration of d-sotalol. The results show that the uniformity of action-potential duration, refractory period, and stimulus-T interval shortening is very high during control and after d-sotalol administration. Shortening dynamics can be described accurately with a biexponential model with pronounced shortening in a very short initial phase and a more gradual shortening with an asymptotic approach to the new steady-state value, in the much longer second phase.

Under normal circumstances the refractory period is shorter than actionpotential duration. During ischemic conditions or high rates in combination with Class IA drugs, postrepolarization refractoriness can occur: due to a decrease in excitability the refractory period prolongs and will outlast action-potential duration. In Chapter 6 the possible consequences of postrepolarization refractoriness for antitachycardia pacing based on stimulus-T interval information were studied. Therefore the decrease in excitability during postrepolarization refractoriness was quantified. It was found that although the relative decrease in excitability was significant the absolute decease in excitability was very limited. Therefore it is very unlikely that these changes will play a role during antitachycardia pacing based on stimulus-T interval information.

The pacing electrode that will be used by the implantable pacemaker during antitachycardia pacing is located in the apex of the right ventricle. The reentry tachycardia however often originates in the left ventricle under ischemic circumstances. These conditions were studied in Chapter 7 , with a tachycardia simulated during the occlusion of the left anterior descending coronary artery. As a result of ischemia, action-potential duration in the left ventricle will shorten, 
whereas action-potential duration in the right ventricle remains unchanged. Nevertheless, the additional amount of action-potential duration shortening and the shortening dynamics induced by the tachycardia are the same for both ventricles. The consequences of this situation for the applicability of antitachycardia pacing based on stimulus- $T$ measurements still need to be addressed.

The first clinical application of antitachycardia pacing using stimulus-T interval information is documented in Chapter 8. The results show that this method of antitachycardia pacing is feasible and safe. Furthermore it is clear that coupling intervals of premature stimuli automatically adapt to the actual refractory period. Coupling intervals, expressed as relative portion of the tachycardia cycle length are longer if the cycle lengths of the tachycardias are shorter.

In Chapter 9 the rational for this new antitachycardia pacing mode is addressed. Especially during fast tachycardias there is need for improvement since conventional antitachycardia pacing modes are not always very successful. Furthermore, there is an increasing need for automatic functions in implantable defibrillators. Antitachycardia pacing based on stimulus-T interval information works automatically and makes complex programming obsolete. The goal of research currently ongoing at the Department of Cardiology of the University Hospital Maastricht is to show that antitachycardia pacing based on stimulus-T interval information is more effective than existing methods. 


\section{Samenvatting}

In Hoofdstuk 1 wordt het kader geschetst waarin dit onderzoek geplaatst kan worden. Er wordt een beknopt overzicht gegeven van de reeks van belangrijke historische ontwikkelingen binnen de cardiale elektrofysiologie. Een van deze ontwikkelingen is de mogelijkheid om in het hart elektrische signalen te registreren en elektrisch te stimuleren. Zo werd er onder meer aangetoond dat de hartritmestoornissen, die kumnen ontstaan na een hartinfarct, met behulp van geprogrammeerde elektrische stimulatie opgewekt en gestopt kunnen worden. De kans dat dit type hartritmestoornis met behulp van antitachycardie stimulatie gestopt wordt, blijkt sterk afhankelijk van de plaats en het moment van stimulatie in relatie tot de cycluslengte van de tachycardie. Wanneer antitachycardie stimulatie door een geimplanteerde stimulator met een elektrode in de rechter hartkamer wordt uitgevoerd is de kans op succes normaal gesproken het grootst als de stimuli meteen na de lokale refractaire periode worden afgegeven. Omdat de werkelijke duur van de refractaire periode tijdens antitachycardie stimulatie niet bekend is, wordt er een schatting gemaakt.

Bijna twintig jaar geleden is een implanteerbare pacemaker ontwikkeld waarmee het mogelijk is de elektrische respons van het hart via de pacemaker elektrode, onmiddellijk na stimulatie te registreren. Daartoe moest de pacemaker worden uitgerust met een laag polariserende elektrode en een stimulatiepuls met een speciale configuratie. Een onderdeel van het geregistreerde signaal is het stimulus-T interval. Dit interval begint op het moment van stimulatie en eindigt in de T-golf van het endocardiale signaal. Het stimulus-T interval geeft daardoor informatie over de duur van de refractaire periode. Een ander belangrijk aspect is dat het stimulus-T interval automatisch en van slag op slag bepaald kan worden door de implanteerbare pacemaker.

Dit principe kan worden gebruikt voor een nieuwe en automatische methode voor antitachycardie stimulatie, waarbij de koppelintervallen van de premature stimuli worden bepaald door stimulus-T interval informatie. Ontwikkeling van deze nieuwe methode voor antitachycardie stimulatie is echter alleen zinvol als de relatie tussen het stimulus- $T$ interval en de refractaire periode tijdens de zeer uiteenlopende omstandigheden waaronder deze techniek wordt toegepast, blijft bestaan.

Om de relatie tussen het stimulus- $T$ interval en de refractaire periode te onderzoeken is een hondenmodel met atrioventrikulair blok gebruikt. Door de lage frequentie van de hartkamers in dit model is het mogelijk een ventrikulaire tachycardie, door een plotselinge en grote verandering van de stimulatiefrequentie, te simuleren. In Hoofdstuk 2 is tijdens een gesimuleerde ventrikulaire tachycardie de relatie tussen het stimulus-T interval en de refractaire periode voor het 
eerst beschreven. De resultaten laten zien dat het verkortingsgedrag van het stimulus-T interval en de refractaire periode als gevolg van de tachycardie uniform is, waarbij de absolute waarde van het stimulus- $T$ interval steeds iets langer is dan van de refractaire periode. Het is dus mogelijk om tijdens een tachycardie met behulp van stimulus-T interval informatie de duur van de refractaire periode te voorspellen.

Omdat een nauwkeurige bepaling van de refractaire periode tijdens de experimenten cruciaal is, worden in Hoofdstuk 3 twee methodes vergeleken die een hoge nauwkeurigheid en reproduceerbaarheid garanderen. De waarden die voor de refractaire periode gevonden worden verschillen niet. Het stimulatie protocol verschilt echter wel. Bij de gebruikelijke methode (decremental scanning) zullen er veelvuldig cycluslengte veranderingen optreden terwijl dat voor de nieuwe methode (incremental scanning) niet geldt. Als het niet gewenst is dat tijdens de bepaling van de refractaire periode ritmestoornissen worden opgewekt is het daarom waarschijnlijk beter om voor incremental scanning te kiezen.

Vervolgens is deze methode in Hoofdstuk 4 gebruikt om na te gaan hoe groot de verschillen in refractaire periode zijn op verschillende tijdstippen in dezelfde hond en onder identieke omstandigheden in verschillende honden. De resultaten tonen aan dat de variatie die gevonden wordt in dezelfde hond op verschillende tijdstippen net zo groot is als de variatie tussen verschillende honden. Het is dus niet mogelijk om de duur van de refractaire periode nauwkeurig te voorspellen op basis van metingen in andere honden of op basis van eerdere metingen in dezelfde hond. Dit benadrukt de noodzaak om tijdens antitachycardie stimulatie de duur van de refractaire periode te bepalen.

In Hoofdstuk 5 wordt het gedrag van het stimulus-T interval, de actie potentiaal duur en de refractaire periode tijdens een gesimuleerde ventrikulaire tachycardie bestudeerd. De metingen worden herhaald na toedienen van d-sotalol. De resultaten laten zien dat de uniformiteit van het verkortingsgedrag van de refractaire periode, de actie potentiaal duur en het stimulus-T interval onder alle omstandigheden zeer hoog is. Verder laten de resultaten zien dat de verkorting volgens een bi-exponentieel patroon verloopt: in een zeer korte eerste fase vindt er veel verkorting plaats, gevolgd door een langere tweede fase waarin de hoeveelheid verkorting per tijdseenheid snel afneemt en tenslotte asymptotisch de nieuwe waarde benadert.

Normaal gesproken is de ventrikulaire refractaire periode korter dan de actie potentiaal duur. Als onder invloed van ischemie of de combinatie van hoge hartfrequenties en klasse IA medicatie de prikkelbaarheid van het weefsel afneemt en daardoor de refractaire periode langer wordt dan de actie potentiaal duur spreekt men van postrepolarization refractoriness. Om de mogelijke gevolgen daarvan voor antitachycardie stimulatie op basis van stimulus-T interval informatie te onderzoeken is in Hoofdstuk 6 gemeten hoeveel de prikkelbaarheid van het myocard afneemt tijdens postrepolarization refractoriness. Alhoewel de relatieve afname in prikkelbaarheid aanzienlijk is, blijven de veranderingen in 
absolute zin zeer beperkt. Het is dan ook zeer onwaarschijnlijk dat deze veranderingen een rol spelen tijdens antitachycardie stimulatie op basis van stimulus- $T$ interval informatie.

De elektrode die door een geimplanteerde stimulator wordt gebruikt voor antitachycardie stimulatie bevindt zich in de apex van de rechter hartkamer. De reentry tachycardieën ontstaan echter in de linker hartkamer, vaak onder ischemische omstandigheden. In Hoofdstuk 7 worden deze omstandigheden bestudeerd. Daartoe wordt een tachycardie gesimuleerd nadat een zijtak van de linker anterior descendens coronair arterie is afgesloten. Door de ischemie neemt de actie potentiaal duur in de linker hartkamer af. De hoeveelheid verkorting en de verkortingsdynamiek van de actie potentiaal duur die vervolgens ontstaat ten gevolge van de tachycardie blijft echter gelijk in de beide hartkamers. De gevolgen hiervan voor de toepasbaarheid van stimulus- $\mathrm{T}$ interval metingen voor antitachycardie stimulatie zullen nog verder onderzocht moeten worden.

De eerste klinische toepassing van antitachycardie stimulatie op basis van stimulus-T interval informatie wordt beschreven in Hoofdstuk 8. De resultaten tonen aan dat de methode werkt en veilig is. Bovendien blijkt dat de koppelintervallen van de premature stimuli worden bepaald door de werkelijke duur van de refractaire periode: naarmate de cycluslengte van de tachycardie afneemt, neemt het relatieve koppelinterval, uitgedrukt als percentage van de cycluslengte, automatisch toe.

In Hoofdstuk 9 wordt nogmaals ingegaan op de beweegredenen om deze nieuwe methode voor antitachycardie stimulatie te ontwikkelen. Vooral tijdens snelle tachycardieën blijken bestaande methodes minder succesvol. Bovendien wordt de behoefte aan automatische functies in implanteerbare defibrillatoren steeds groter. Antitachycardie stimulatie op basis van stimulus- $\mathrm{I}$ informatie werkt automatisch en kan ingewikkelde programmering overbodig maken. Het onderzoek dat op dit moment binnen de vakgroep Cardiologie van het Academisch Ziekenhuis Maastricht wordt uitgevoerd is erop gericht om de effectiviteit van deze nieuwe vorm van antitachycardie stimulatie aan te tonen. 


\section{Dankwoord}

Dit dankwoord is de afsluiting van een enerverende periode in mijn leven. Een periode waarvan het resultaat in de vorm van dit proefschrift nu voor iedereen duidelijk zichtbaar is. Het moge duidelijk zijn dat dit proefschrift niet het resultaat is van de inspanningen van één persoon. Veel mensen hebben elk op hun eigen wijze bijgedragen tot dit proefschrift. We mogen trots zijn op het resultaat. Ik wil van deze gelegenheid gebruik maken om iedereen daarvoor zeer uitdrukkelijk te bedanken.

Daarnaast is er ook een groep mensen die ik met naam wil noemen omdat hun bijdrage een speciaal en persoonlijk woord van dank verdient.

De eerste man die ik wil bedanken is Prof. Hein Wellens. Tijdens zijn lange carrière is reeds zo veel eerbetoon en lof over hem uitgestrooid dat ik daar nauwelijks nog iets aan kan toevoegen. Toch is er iets waar ik aan denk als ik terugkijk op de afgelopen jaren. Toen ik voor het eerst aan $u$ werd voorgesteld was $u$ voor mij een professor die de dingen deed die een professor pleegt te doen. Later toen ik een idee van de omvang van uw kennis en uw bijdrage aan het vakgebied kreeg bent u de professor geworden. Tenslotte heb ik me gerealiseerd wat het betekent om Hein Wellens als promotor te hebben: $\mathrm{u}$ bent mijn professor geworden.

Met Dr. Marc Vos heb ik de meeste uren aan dit proefschrift besteed. Beste Marc, je hebt een omgeving gecreëerd waarin je kan leren onderzoeken en waarin uitstekend onderzoek gedaan wordt. Ik heb ervaren dat onderzoeken een vak is waarvoor hard gewerkt moet worden en veel doorzettingsvermogen nodig is. Gedurende onze lange samenwerking zijn we het niet altijd met elkaar eens geweest, maar we zijn telkens weer in staat gebleken om een nieuw hoofdstuk aan dit proefschrift toe te voegen. Ik ben ervan overtuigd clat veel mensen gedacht hebben dat dit proefschrift er nooit zou komen. Omdat ik wist dat jij altijd mijn gesprekspartner zou blijven heb ik er nooit aan getwijfeld. Ik hoop dat we onze samenwerking in de toekomst kumnen voortzetten.

De klinische fase van dit onderzoek werd mede begeleid door Dr. Joep Smeets. Beste Joep, ik wil je langs deze weg bedanken voor de belangrijke klinische inbreng. De omvang van jouw kennis van klinische electrofysiologie is me tijdens een 2 uur durende treinreis zonder semafoononderbreking pas echt duidelijk geworden. Het doet me dan ook genoegen dat het "Stimulus-T" project doorgaat. Als de verschillende partners in het project hun medewerking blijven verlenen moet het mogelijk zijn om aan te tonen dat antitachycardie stimulatie op basis van stimulus-T interval informatie de beste methode voor antitachycardie stimulatie is.

Dat ik Dr. Karel den Dulk vanuit deze positie wil bedanken is voor mij heel vanzellsprekend. Karel was de eerste cardioloog waarmee ik kennis mocht maken toen ik het Academisch Ziekenhuis in Maastricht betrad. Van Karel heb ik veel 
geleerd, hij was iemand met creatieve ideeën. Zijn idee is de basis geweest voor dit onderzoek. Helaas heeft Karel grote delen van dit werk van de zijlijn moeten bekijken, hij was gedurende de laatste jaren van zijn leven niet meer in staat om zijn creatieve geest en zijn eens zo grote werkkracht te gebruiken. Hij heeft heel veel mensen geholpen maar was niet in staat om zichzelf te laten helpen. Karel, op de dag dat ik hoop te promoveren is het alweer een jaar geleden dat we afscheid van je hebben genomen. Ik zal je niet vergeten.

De leden van de beoordelingscommissie wil ik bedanken omdat zij een deel van hun zeer schaarse tijd hebben willen gebruiken om mijn manuscript te beoordelen en voor hun oppositie tijdens de openbare verdediging van dit proefschrift. Ik ben vereerd dat Prof. Maurits Allessie die ik meer dan 15 jaar geleden tijdens mijn H.T.S. stage in het oude Biomedisch Centrum leerde kennen, de voor[150] zitter van mijn beoordelingscommissie wilde zijn. Ook Prof. Rob Reneman ken ik uit die tijd. Hij is iemand die achter veel meer deuren staat clan ik toen kon vermoeden. Hij is in staat om met oprechte aandacht en waardering alle leden van een team te motiveren. Prof. Theo Arts is mijn begeleider geweest tijdens mijn H.T.S. stage en later tijdens mijn aanstelling bij de vakgroep Biofysica. Het is in deze omgeving dat mijn belangstelling voor cardiologie en met name electrofysiologie is ontstaan. Het doet me veel genoegen dat onze wegen elkaar nog eens kruisen. Dat Prof. Luc Jordaens lid is geworden van de beoordelingscommissie doet me veel plezier. Ik weet dat hij in zijn overladen agencla nauwelijks de tijd heeft kunnen vinden om mijn manuscript te beoordelen. I am very pleased that Prof. Trappe was willing to review the manuscript of my thesis. As one of the leading experts in electrophysiology and antitachycardia pacing therapy his contribution is highly estimated.

Arno Muijtjens (Medische Informatica, Universiteit Maastricht), Ben Vonk en Malcolm Begemann (Vitatron Medical B.V., Dieren), Richard Houben, Volkert Zeylemaker en Fred Lindemans (Medtronic Bakken Research Center, Maastricht) wil ik bedanken voor hun bijdrage aan dit onderzoek.

De hulp van mijn paranimfen Roy Jongen en Paul Volders is onontbeerlijk geweest. Gelukkig heb ik het schrijven van dit dankwoord lang uitgesteld. Daardoor weet ik nu uit eigen ervaring hoe belangrijk de steun van paranimfen is. Roy is niet alleen in staat geweest om alle voorwaarden in kaart te brengen waaraan voldaan moeten worden om mijn promotie tot een succes te maken, hij heeft er ook voor gezorgd dat aan al die voorwaarden voldaan kan worden. Roy, ik ben blij dat je al die zaken zo uitstekend voor me geregeld hebt. Het was voor mij een eer om paranimf te mogen zijn tijdens de promotie van Paul, een 'cum laude' promotie die het begin zal zijn van een belangrijke wetenschappelijke carrière. Het is voor mij dan ook een hele geruststelling dat Paul me terzijde zal staan tijdens mijn promotie. Hij kent de klinisch wetenschappelijke ongeving en zal ervoor zorgen dat alle geschreven en ongeschreven regels in acht worden genomen. Paul, ik hoop dat je steeds in staat zal zijn om een zo goed mogelijk evenwicht te vinden tussen je professionele ambities en je gezin. Ilja en Samuel zullen je daarbij zeker helpen. 
Binnen de groep Experimentele Cardiologie zijn Jet Leunissen en Roel Spätjens de steunpilaren waar je op kan bouwen. Jet, ik heb bewondering voor je werkhouding, al heel veel jaren ben je een vaste waarde binnen de groep. Zells zware tegenslagen in je leven laat je niet ten koste gaan van je werk. Roel, de enkele keren dat ik een beroep op je heb gedaan voor het maken van een poster hebben me geleerd dat je van het goede hout gesneden bent. Ik denk dat het belang van jullie bijdrage door velen wordt onderschat.

Mijn collega's van het eerste uur Marieke de Groot en Cora Verduijn hebben inmiddels ook hun weg (naar Amby) gevonden. Marieke, ik kan me nog goed onze eerste ontmoeting tijdens een etentje herinneren, je had het toen koud, maar dat is wel vaker zo. Ik had toen geen idee dat jij met zoveel energie en doorzettingsvermogen aan je toekomst wilde werken. Inmiddels woon je, samen met Maurits, in ons mooie dorp en hebben jullie iets opgebouwd waar veel mensen jaloers op mogen zijn. Cora, jij weet steeds weer de data te vinden die voor een ander onvindbaar lijken. Mede daardoor en door je vasthoudendheid en vastberadenheid kun je steeds op jouw eigen manier je doelen bereiken.

De professionele opmaak van dit proefschrift werd verzorgd door mijn zus Peggy Bisschoff. Haar wil ik bedanken voor haar geduld en begrip voor niet gehaalde deadlines en natuurlijk mijn excuses voor de verwaarlozing van mijn andere zus Madeleine tijdens haar finale inspanningen. Jullie ouders Jan en Marian wil ik bedanken voor alle gastvrijheid en zapjes en hun oprechte betrokkenheid.

Alle dames van het secretariaat wil ik bedanken voor hun hulp en voor hun belangstelling. Met name Monique wil ik veel geluk wensen met de aanstaande gebeurtenissen. Zonder de Instumentele Dienst en de Centrale Proefdier Voorzieningen was dit onderzoek niet mogelijk geweest. Bedankt voor de deskundige ondersteuning.

Verder zijn er nog veel mensen die hebben bijgedragen tot het welslagen van mijn promotietijd. Jurren (carpe diem en natuurlijk die woorden van Paul); Dirk (betrouwbaar en zuverlässig); Marieke (tijd voor idealen); Mirella (lang niet meer gezien); Jash, Ashis en Ravinder (India op zijn best); Domien (fiets met drie kinderzitjes en zware rugzak); Pieter (koga 66 inch, nature); Jérôme (Weissenburger?); Frits (LV pacing met stip, apple?); Maaike (blijven schrijven); Jolanda (op zoek); Xander (sony, powerpoint en circulation); Pim ( $\mathrm{ja}$, ik heb een datum); Rob en Berto (heel belangrijk); Ferenc (jammer); Attila (difficult choices); Tamas (the best stories); Theo, Ruud en Jo (voor de broodnodige koffie en variatie); Leon (jammer, prima instrumentele dienstverlener); Loes (voor de laatste loodjes); Pee, Marc, Jela, Kirsten, Gerard (slachtoffers van de tijdelijke sociale verarming); Gerard (want er is meer); John (voor cle betere ( $\mathrm{Ni}$ ) cols).

Mijn collega's bij St Jude Medical Nederland B.V. wil ik bedanken voor het begrip en de medewerking op momenten dat ik er niet was omdat er weer iets af moest. 
Mijn schoonouders, Sjaak en Mia, wil ik bedanken voor de vele uren die ze van me hebben overgenomen, hun voortdurende belangstelling voor mijn werk, de kinderschoentjes en natuurlijk voor hun dochter.

Mam en pap wil ik bedanken voor de 38 jaar die ze aan de totstandkoming van dit proefschrift hebben bijgedragen. Jullie hebben me geleerd dat je iets waaraan je begint ook moet afmaken. We mogen "stolz" zijn op het resultaat. Ruud wil ik bedanken voor de gezellige bezoekjes en het "knokken".

Casper en Eeke zullen er wellicht aan moeten wennen als papa wel weer mee gaat en niet in de kelder zit. Ik ben blij dat jullie er zijn en dat ik door jullie onbevangen en heldere blik de werkelijk waardevolle zaken van het leven nog steeds ken.

Marlie, wij werken samen aan onze toekomst, waarbij ieder een belangrij-

[152] ke bijdrage levert. Dit proefschrift is een onderdeel van het geheel waaraan jij net zo hard werkt als ik. Dat de buitenwereld daar op momenten als dit een ander beeld van krijgt doet jou te kort. Ik wil je bedanken voor je onvoorwaardelijke steun en de ruimte die je hebt gecreëerd om deze taak te volbrengen. 


\title{
Curriculum Vitae
}

\author{
Name Henny (Hendrik Marie) Leerssen \\ Born Simpelveld, The Netherlands, May 21, 1962 \\ Nationality Dutch \\ Marital Status Married with Marlie Nillesen, father of Casper and Eeke
}

Education
$1974-1980 \quad$ V.W.O. $\beta$, Scholengemeenschap Sancta Maria, Kerkrade-West 1980-1985 Hogeschool Heerlen, Department of Technical Physics, BS
$1992-1997$ Ph.D. student at the Department of Cardiology, Cardiovascular Research Institute, Maastricht University, The Netherlands.
Supervisors: Prof. dr. H.J.J. Wellens, Dr. M.A. Vos, and Dr. K den Dulk.

\section{Working experience}

$\begin{array}{ll}1985-1987 & \text { Research assistant, Department of Biophysics, Maastricht } \\ & \text { University } \\ 1987-1992 & \text { Heart- and Lungfunction Department, University Hospital } \\ & \text { Maastricht } \\ 1997- & \text { Product Specialist and Scientific Advisor, St Jude Medical } \\ & \text { Nederland B.V. }\end{array}$




\section{Publications}

\section{Abstracts}

1. Leerssen HM and den Dulk K. Data transmission with a modem to the central pacemaker patient registration. Neth J Cardiol 1990; 3:163.

2. Leerssen HM, den Dulk K, Vos MA, van der Zande J, Begemann MJS, and Wellens HJJ. Validation of the stimulus- $T$ interval for antitachycardia pacing: importance of the individual assessment. Pflügers Arch 1993; 424:R6.

3. Leerssen HM, den Dulk K, Vos MA, van der Zande J, Begemann MJS, and Wellens HJJ. Individual variation in dynamic behaviour of ventricular effective refractory period and action-potential duration following rate changes as assessed by the stimulus-I technique. Eur Heart J 1993; 14(suppl):87.

4. Leerssen HM, Vos MA, den Dulk K, van der Zande J, Begemann MJS, and Wellens HJJ. Inter- and intraindividual variations in shortening of ventricular effective refractory period after an abrupt clecrease in pacing cycle length. Eur J C P E 1994; 4:148.

5. Leerssen HM, Vos MA, den Dulk K, van der Zande J, and Wellens HJJ. Is the ventricular effective refractory period different when determined by incremental versus decremental scanning?: the effect of pacing cycle length, d-sotalol and levcromakalim. Eur J C P E 1994; 4:152.

6. Leerssen HM, Vos MA, den Dulk $\mathrm{K}$, van der Zande J, Begennann MJS, and Wellens HJJ. Feasibility of a dynamic and individually suited antitachycardia pacing mode based on real-time stimulus-T information. Cardiologie 1994; 7/8:300.

7. Leerssen HM, Vos MA, den Dulk K, van der Zande J, and Wellens HJJ. The relevance of the diastolic threshold for post repolarization refractoriness. Pflügers Arch 1995; 430(suppl):R112.

8. Leerssen HM, Vos MA, den Dulk $\mathrm{K}$, van der Zande J, and Wellens HJJ. A low dose of levcromakalim is not pro-arthythmic in dogs subjected to repeated episodes of ischemia. J Mol Cell Cardiol 1996; 28:A55. 
9. Dassen WRM, Postma EO, Vercluyn SC, Leunissen HDM, Leerssen HM, and Vos MA. Do torsades de pointes tachycardia and ventricular fibrillation represent chaotic arrhythmias that can be differentiated using non-linear analysis? Eur J C PE 1996; 6:28.

10. van Opstal J, Verduyn SC, Vos MA, Leerssen HM, Leunissen HDM, and Wellens HJJ. Simple measurement of JTU-area on the ECG indicates changes in interventricular dispersion of repolarisation in dogs. J Am Coll Cardiol 1998; 31 no.2(suppl A):253.

11. Leerssen HM, Vos MA, Houben R, den Dulk K, and Wellens HJJ. Transient ischemia does not alter dynamics of action-potential duration shortening after an

[156] abrupt decrease in pacing cycle length in the dog. PACE 1999; 22(Pt. II):A31.

12. Leerssen HM, Smeets JLRM, Vos MA, Zeylemaker VA, den Dulk K, and Wellens HJJ. Application of the stimulus-I interval for antitachycardia pacing. Europace 2000; 1(suppl B):B10.

\section{Journal Articles}

1. Leerssen HM. Standaardisatie van pacemaker connectors. Techniek in de gezondheidszorg 1990; 4:39-40.

2. den Dulk $K$, Leerssen HM, Vos MA, Leunissen HDM, Dassen WRM, Kersemakers J, Begemann MJS, and Wellens HJJ. Applicability of the stimulusinterval for antitachycardia pacing. PACE 1991; 14(Pt. II):1757-1761.

3. Dassen WRM, Mulleneers RGA, and Leerssen HM. Elektrische stimulatie in de cardiologie; diagnose en therapie. Klinische Fysica 1993; 4:187-191.

4. Leerssen HM, Vos MA, den Dulk K, van der Zande J, Begemann MJS, and Wellens HJJ. Inter- and intraindividual variations in shortening of ventriculat effective refractory period after an abrupt decrease in pacing cycle length. PACE 1994; 17(Pt. II):2079-2083.

5. Leerssen HM, Vos MA, den Dulk K, van der Zande J, and Wellens HJJ. Is the ventricular effective refractory period different when determined by incremental versus decremental scanning?: the effect of pacing cycle length, d-sotalol and levcromakalim. PACE 1994; 17(Pt. II):2084-2089. 
6. Leerssen HM, Vos MA, den Dulk K, van der Zande J, Muijtjens AMM, Begemann MJS, and Wellens HJJ. Steady-state and dynamic behavior of ventricular repolarization and refractoriness in the dog: the effect of multiple cycle-length changes and d-sotalol administration. PACE 1998; 21:1766-1777.

7. Leerssen HM, Vos MA, den Dulk K, van der Zande J, and Wellens HJJ. Ratedependent effects of procainamide on the threshold current for pacing in the setting of post repolarization refractoriness in dogs. PACE 1999; 22:291-301.

8. Leerssen HM, Vos MA, Houben R, den Dulk K, and Wellens HJJ. High uniformity of left and right ventricular repolarization dynamics induced by an abrupt decrease in pacing cycle length in a dog is not affected by left-ventricular ischemia. J Carcliovasc Electrophysiol 2000; 11:421-429.

\section{Book Chapters}

1. Reneman RS, Allessie MA, Arts T, Augustijn CH, Leerssen HM, and Prinzen FW. Electrical and mechanical mapping of the left-ventricular free wall during normoxia and regional myocardial ischemia - the effect of electrical stimulation. In Sideman S, Beyar R (eds.): Analysis and Simulation of the Cardiac System Ischemia. Boca Raton, Floricla, USA, CRC Press, Inc.; 1989:21-35.

\section{Patents}

1. Begemann MJS, den Dulk K, Leerssen HM, and Zeylemaker VA. System and method for inducing tachycardia. United States Patent 6,052,621 April 18, 2000. 
